\title{
DEVELOPMENT OF AN MRI-BASED WORKFLOW FOR POST- IMPLANT DOSIMETRY OF PROSTATE LOW-DOSE-RATE (LDR) BRACHYTHERAPY
}

\author{
by \\ Reyhaneh Nosrati, MSc, Ryerson University, 2015
}

\begin{abstract}
A dissertation
presented to Ryerson University

in partial fulfillment of the requirements for the degree of

Doctor of Philosophy

in the program of

Biomedical Physics
\end{abstract}

Toronto, Ontario, Canada, 2019

(C) Reyhaneh Nosrati, 2019 


\section{Declaration}

I hereby declare that I am the sole author of this dissertation. This is a true copy of the thesis, including any required final revisions, as accepted by my examiners.

I authorize Ryerson University to lend this thesis to other institutions or individuals for the purpose of scholarly research.

I further authorize Ryerson University to reproduce this thesis by photocopying or by other means, in total or in part, at the request of other institutions or individuals for the purpose of scholarly research.

I understand that this thesis may be made electronically available to the public.

Reyhaneh Nosrati 


\title{
DEVELOPMENT OF AN MRI-BASED WORKFLOW FOR POST- IMPLANT DOSIMETRY OF PROSTATE LOW-DOSE-RATE (LDR) BRACHYTHERAPY
}

\author{
Reyhaneh Nosrati \\ PhD, Biomedical Physics, Ryerson University, 2019
}

\begin{abstract}
Permanent implantation of low-dose-rate (LDR) brachytherapy seeds is a well-established treatment modality for patients with localized prostate cancer. The quality of the implant is assessed within 30 days following implantation through post-implant dosimetry. The standard recommended procedure for post-implant dosimetry is based on computed tomography (CT). CT provides excellent seed visualization and localization; however, due to poor soft tissue contrast and challenging anatomical identificatio, $n$ it leads to significant interobserver variabilities. The current MRI-CT fusion-based workflow for post-implant dosimetry LDR prostate brachytherapy takes advantage of the superior soft tissue contrast of MRI but still relies on CT for seed visualization and detection, and it suffers from image fusion uncertainties and extra cost and logistics. The lack of positive contrast from brachytherapy seeds in conventional MR images remains a major challenge towards an MRI-only workflow for post-implant dosimetry of LowDose-Rate (LDR) brachytherapy.
\end{abstract}

In this thesis, a clinically feasible MRI-based workflow has been developed for brachytherapy seed visualization and localization. The seed visualization is based on a novel Quantitative Susceptibility Mapping (QSM) algorithm. The proposed seed localization on QSM 
utilizes machine learning algorithms. The reliability of the proposed workflow has been validated on 23 patients by comparing the seed positions and final dosimetric parameters between the proposed MRI-only workflow and the clinical CT-MRI fusion-based approach and there was excellent agreement between the two methods. 


\section{ACKNOWLEDGMENTS}

My sincerest appreciation goes to my amazing supervisors Professors Greg J. Stanisz and Ana Pejović-Milić, for their scientific and personal guidance during my Ph.D. study. It has been a great honour to be a Ph.D. student under their supervision. Greg is the kind of person you will instantly love and never forget once you meet him. He is the coolest advisor and one of the smartest people I have ever known. He knows MRI by heart and was the only person who always had the best answers to all my MRI related questions. The joy and enthusiasm he has for his research were contagious and motivational for me, even during tough times. Without his generosity to share his encouragement, or more importantly, criticism, it would not have been possible for me to finish this work. Ana has been my role model since I met her for the first time when I started my MSc at Ryerson University. She has always been very encouraging and supportive and the best example of a successful woman physicist and professor. I appreciate all their contributions of time, ideas, and funding to make my Ph.D. experience productive and stimulating.

I am deeply grateful to my amazing clinical advisor Dr. Gerard Morton for his constant level of encouragement, support, and dedication to this project. He is a professor of medicine and a world-leading brachytherapy expert who was the best person to challenge my project from the clinical perspective. He helped me establish collaboration with other radiation oncologists at Sunnybrook Health Sciences Centre and made the clinical transition of the developed workflow smooth and possible. His scientific inputs to this work significantly improved the overall performance of the developed algorithm. 
I also thank Dr. Matt Wronski for his help with using MIM software stations to analyze my results on a clinical platform. He helped me to come up with the best process to validate my clinical MR-based results with the standard CT-based approach after several hours of insightful discussion.

I also thank my former supervisor Dr. William Song, for defining such an amazing project and trusting in me. William was a very good friend, also a very encouraging and inspiring advisor. I hope that I could be as lively, enthusiastic, and energetic as William. I'm also thankful to Dr. Abraam Soliman for his support and help from the very first steps of this project when I was new to the MRI world. I could not imagine my research field transition from optics in MSc to MRI without his help. His enthusiasm and willingness to share his deep knowledge of MRI with me was very encouraging and I'm deeply grateful for that. I also like to thank Dr. Masoud Hashemi for opening the window to the fascinating world of machine learning. He was the person who brought the idea of implementing machine learning algorithms in this project and his scientific advice in the area of machine learning during this project are very much appreciated. Thank you.

I will forever be thankful to my former MSc supervisor, Dr. Vladislav Toronov. Vlad was the person who taught me how a research project should be planned and executed to achieve the desired outcome with minimal waste of time and resources. I started writing medical papers in my graduate study as a MSc student and it was a nightmare for a person cultivated for engineering and Physics. Vlad's help and insightful comments enormously helped me to handle this shift in focus; this valuable skill helped me to write several papers and abstracts for a wide range of audience from physicians to physicists and engineers.

A good support system is important to surviving and staying sane in grad school. I was very lucky that my research was based at Sunnybrook Health Sciences Center (SHSC). The support I 
received from the excellent Medical Physics team at SHSC had a great influence on my research progress. I'm very grateful to Drs. Ananth Ravi, Claire McCann, Alex Karotki, Moti Paudel and Melanie Davidson for their support and valuable inputs to this thesis.

I also thank the members of my PhD supervisory committee members, professors Yuan Xu and Nilesh Ghugre for their helpful career advice and suggestions in general.

I am deeply thankful to our lab members Dr. Wilfred Lam and Dr. Wendy Oakden for providing unconditional support and assistance.

In regard to my methodology development and primary validations on phantom, I'm thankful to my industrial collaborators, IsoAid, CIVCO and Carbon Medical Technologies for providing brachytherapy seeds and fiducial markers at no cost.

I'm deeply thankful to my beloved sister Roya for her help with making high quality graphics for my presentations and helping with preparing and labeling my training data set for supervised machine learning algorithm. She has been my best friend my entire life and she has always been there for me. I would like to thank my parents for all their love and encouragement. And most of all for my loving, supportive, encouraging, and patient husband, Mehdi. Thank you.

Finally, I would like to acknowledge the members of my examination committee, Drs. Alex MacKay, Javad Alirezaei, and James Grafe, who agreed to act as official examiners of this thesis. 
I dedicate this thesis to my parents, my husband, Mehdi, and my beloved cat, Belfy for their constant support and unconditional love.

I love you all dearly. 


\section{TABLE OF CONTENTS}

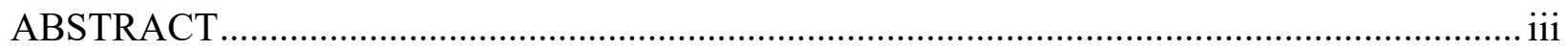

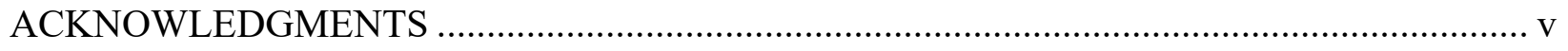

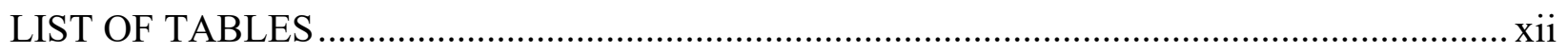

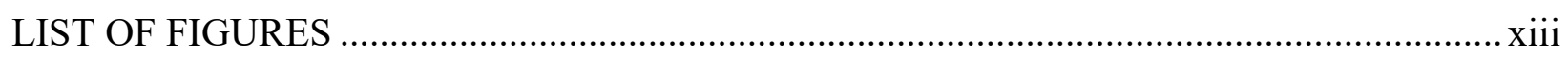

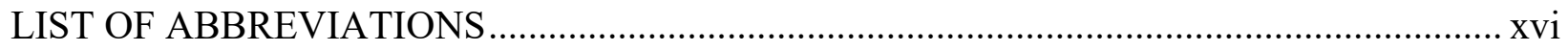

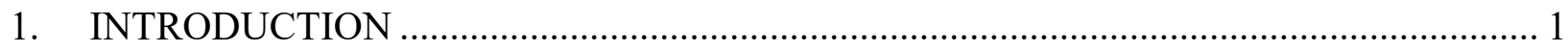

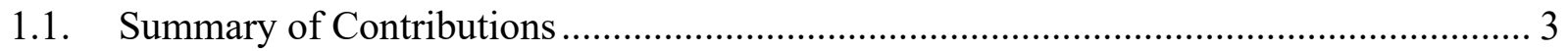

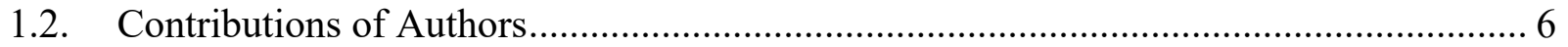

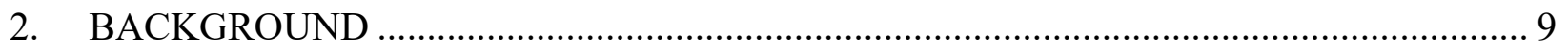

2.1. Low Dose Rate Brachytherapy .......................................................................... 9

2.2. Previous Works in MRI-based Post-Implant Dosimetry............................................... 13

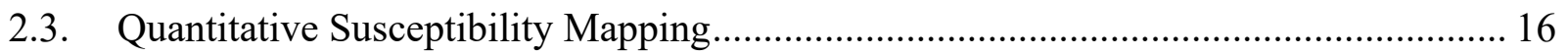

2.4. Machine Learning for Medical Image Analysis......................................................... 19

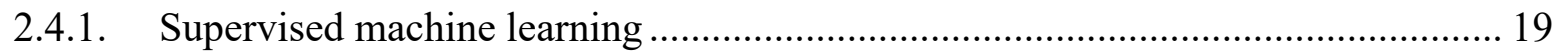

2.4.2. Unsupervised machine learning ...................................................................... 20

3. MRI-BASED AUTOMATED DETECTION OF IMPLANTED LOW DOSE RATE (LDR) BRACHYTHERAPY SEEDS USING QUANTITATIVE SUSCEPTIBILITY MAPPING (QSM) AND UNSUPERVISED MACHINE LEARNING (ML) ............................................ 22

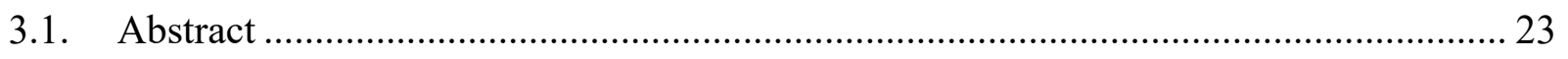

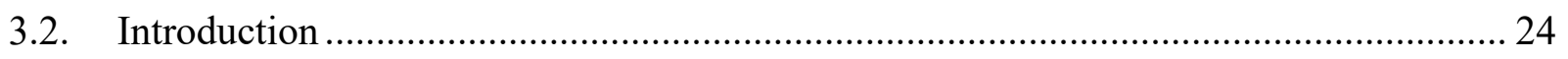

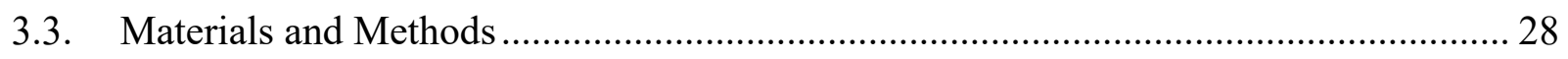

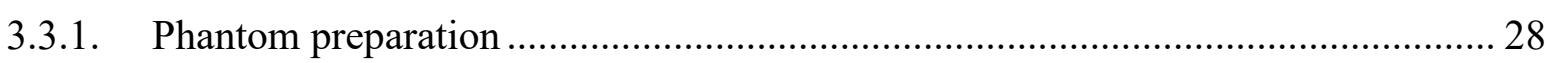

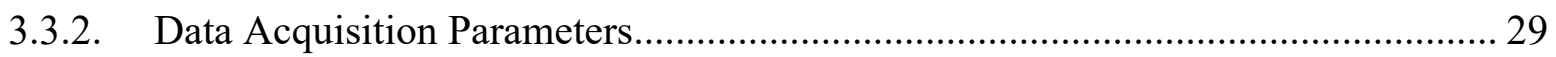

3.3.3. Calculation of 3D Magnetic Susceptibility Maps .................................................. 30

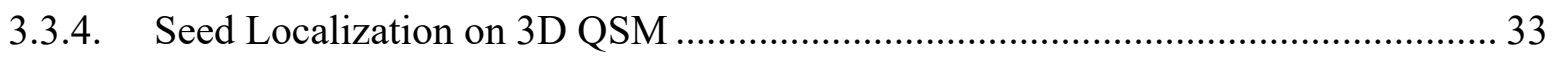

3.3.5. Result Validation and Statistical Analysis ............................................................ 35

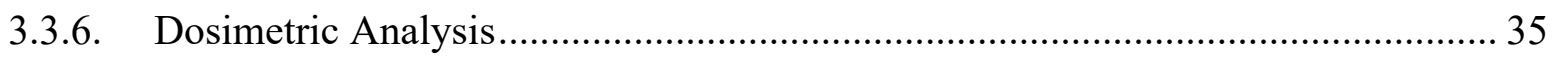

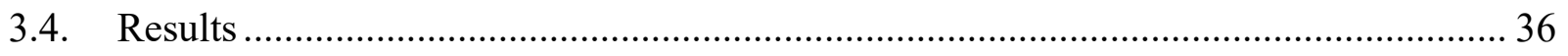

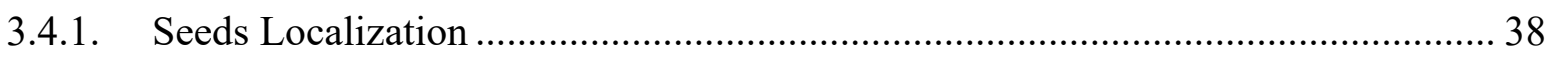

3.4.2. Performance of the proposed workflow on MR data acquired by $1.5 \mathrm{~T}$ scanner .... 40

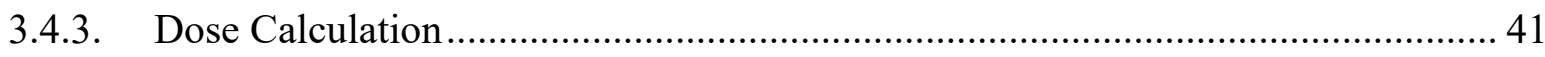

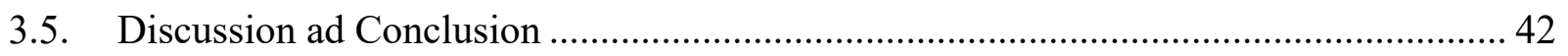

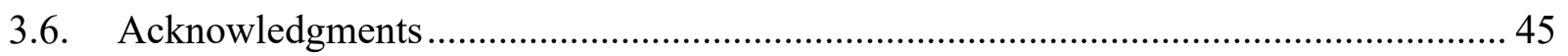


4. FEASIBILITY OF AN MRI-ONLY WORKFLOW FOR POST-IMPLANT DOSIMETRY OF LOW-DOSE-RATE (LDR) PROSTATE BRACHYTHERAPY IN PRESENCE OF

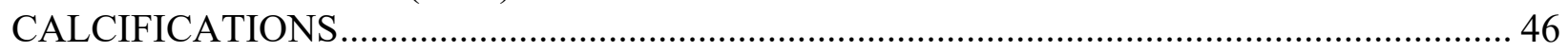

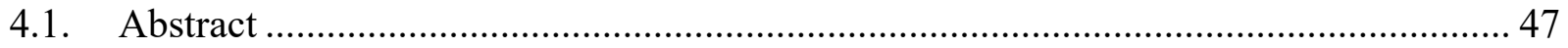

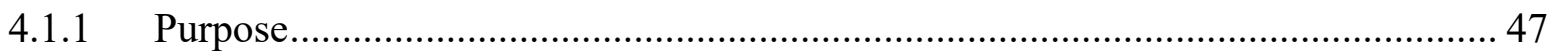

4.1.2 Materials and Methods............................................................................ 47

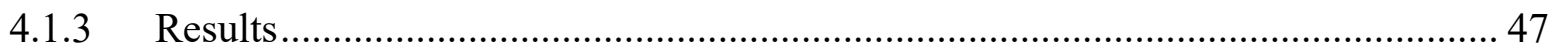

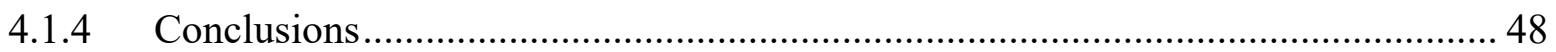

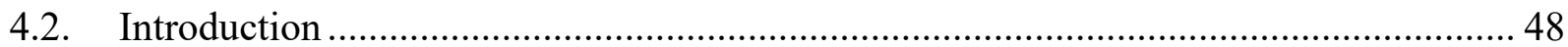

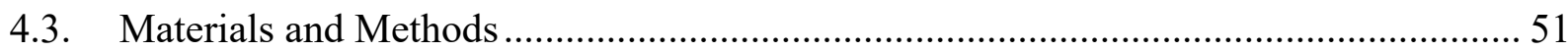

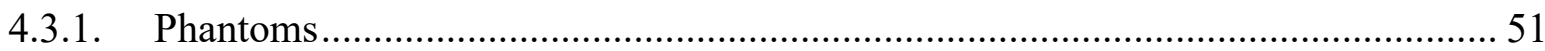

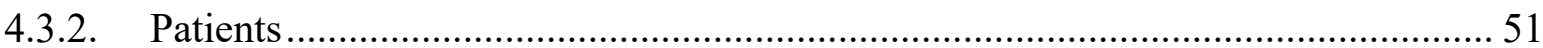

4.3.3. Data Acquisition Parameters....................................................................... 52

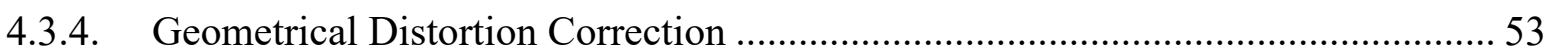

4.3.5. Quantitative Susceptibility Mapping (QSM) using MEDI+0 ............................ 54

4.3.6. Seed Localization and Dosimetric Comparison............................................... 57

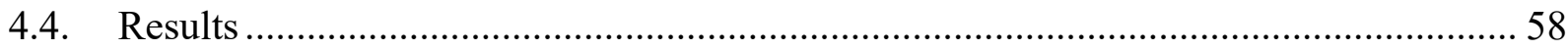

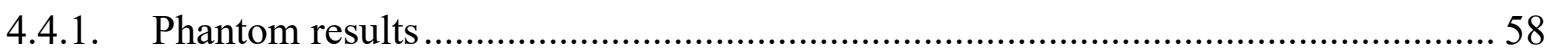

4.4.1.1. Seed Visualization at Different Orientations with Respect to B0 .................... 58

4.4.1.2. MR-based Seed Localization and Dosimetric Analysis .................................. 59

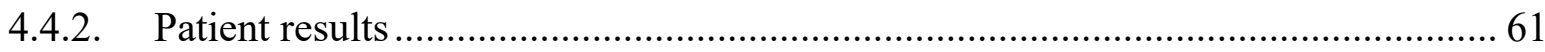

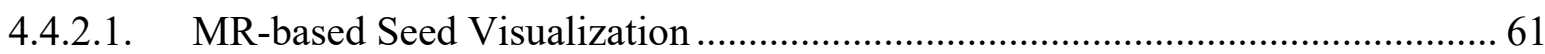

4.4.2.2. MR-based Seed Localization and Dosimetric Analysis .................................. 65

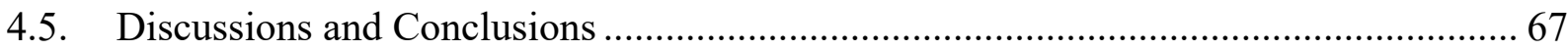

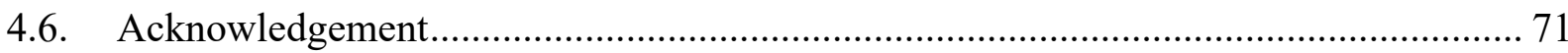

5. POST-IMPLANT DOSIMETRY OF PERMANENT PROSTATE BRACHYTHERAPY: COMPARISON OF QSM-BASED MRI-ONLY AND CT-MRI FUSION WORKFLOWS ...... 72

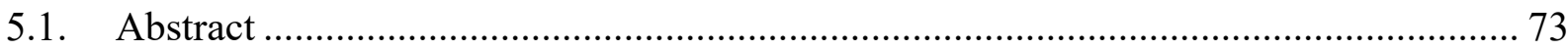

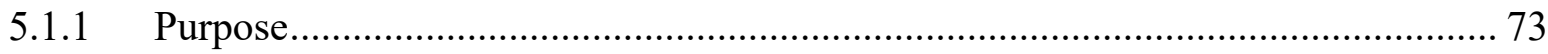

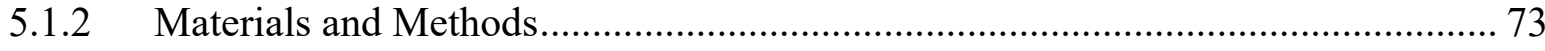

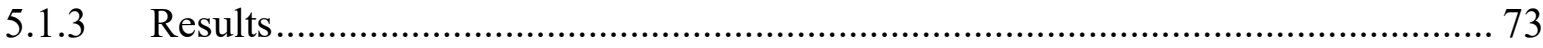

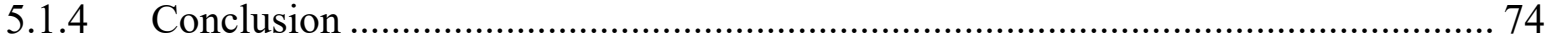

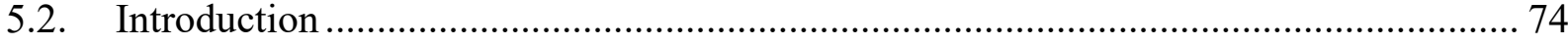

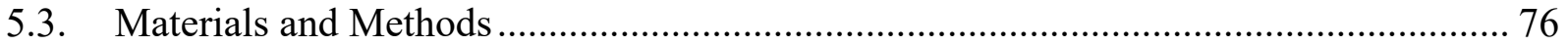

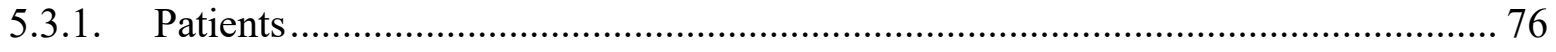




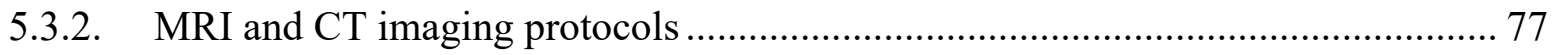

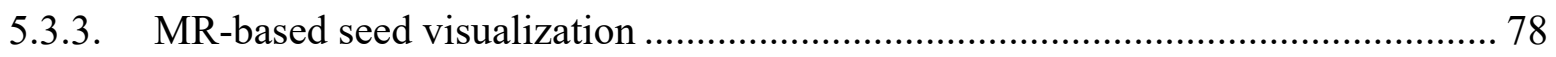

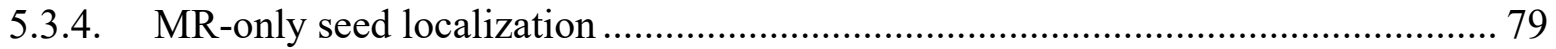

5.3.5. CT-MRI versus MR-only seed localization and dosimetry …………………….... 81



5.4.1. MR-based Positive Contrast Seed Visualization ..................................................... 82

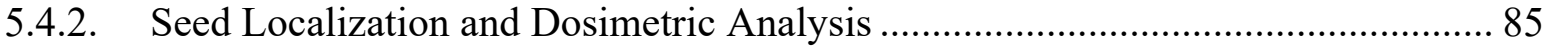

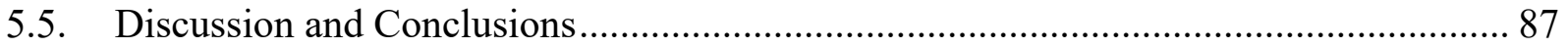

6. POTENTIAL APPLICATIONS OF THE QUANTITATIVE SUSCEPTIBILITY MAPPING (QSM) IN MR-GUIDED RADIATION THERAPY ………………………........... 91

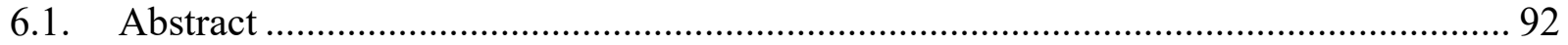

6.1.1. Background and Purpose ............................................................................... 92

6.1.2. Materials and Methods................................................................................ 92

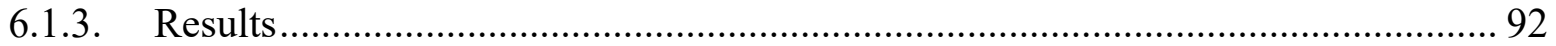

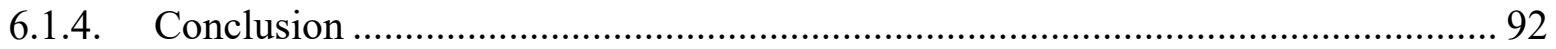

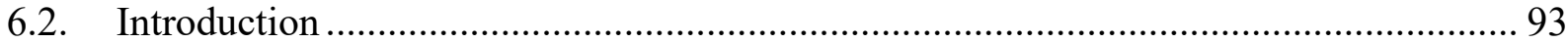

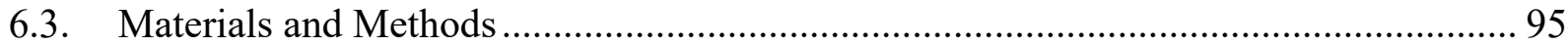

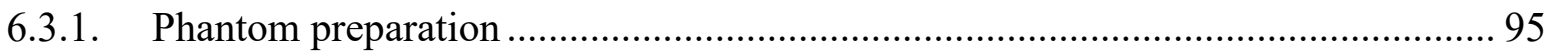

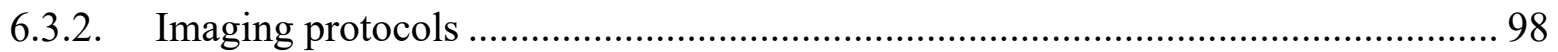

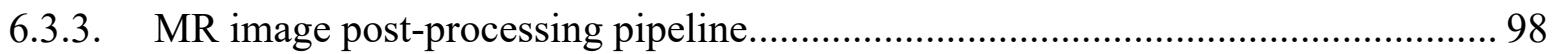

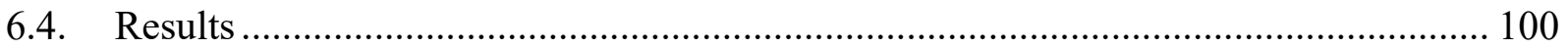

6.4.1. Effect of seed type and nearby calcifications ................................................ 100

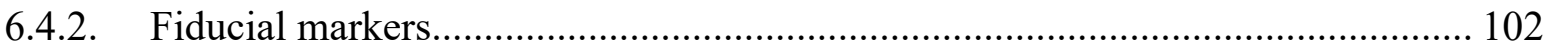

6.4.3. HDR interstitial brachytherapy needles, markers and obturators .......................... 104

6.4.4. Performance comparison between the MEDI and MEDI+0................................ 106

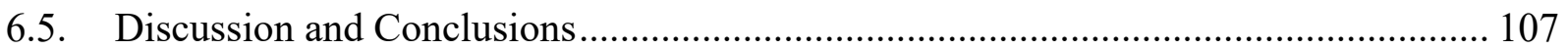

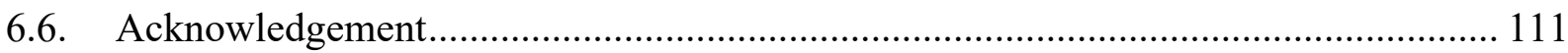

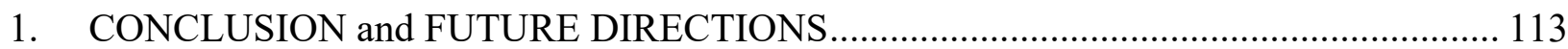

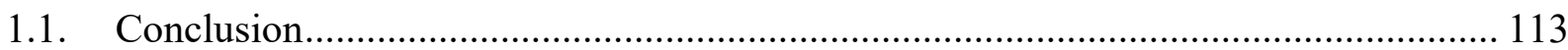

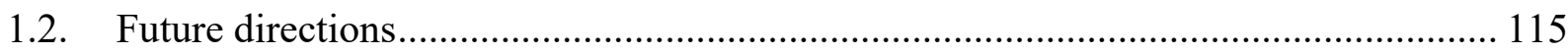

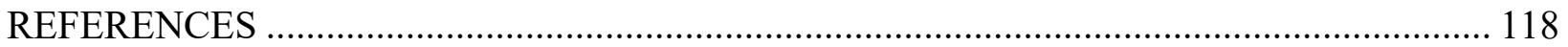




\section{LIST OF TABLES}

Table 4.1; The summary of dosimetric parameters obtained from DVH curves calculated using

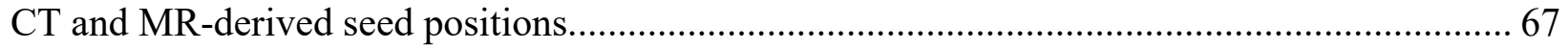

Table 5.1; Patient characteristics and brachytherapy treatment planning parameters .................. 77

Table 6.1; Volume magnetic susceptibility and atomic number of the studied materials............ 97

Table 6.2; The average estimated magnetic susceptibility values for different investigated devices/materials. 106 


\section{LIST OF FIGURES}

Figure 2.1; LDR brachytherapy seed sample configuration for I-125 and Pd-103 radionuclides 10

Figure 3.1; (a) The constructed phantom containing 20 seeds (in 5 strands) and three calcifications. MIP reconstruction of the CT image obtained while the phantom axis (along the B0 arrow) was (b) perpendicular (c) parallel to the CT bed. MIP reconstruction of (d) 3T MR magnitude image at $\mathrm{TE}=6.5 \mathrm{~ms}$; (e) $3 \mathrm{~T}$ MR phase image at $\mathrm{TE}=6.5 \mathrm{~ms}$; (f) phase image after temporal unwrapping and background field removal using PDF method; (g) The estimated off resonance frequency map; (h) the estimated susceptibility map. The B0 arrow indicates the direction of the MR static magnetic field. 38

Figure 3.2; (a) the point cloud representation of the thresholded QSM; (b) the calculated k-means based Silhouette values as a function of number of clusters; (c) the estimated medoid (centroids) and orientation of each cluster (seed), the dashed lines represent the actual orientation of each seed strand.

Figure 3.3; The output of the CT-based VariSeed seed finder software for different user-defined number of seeds (a) 20 seeds, parallel scan; (b) 20 seeds, perpendicular scan; (c) 14 seed; (d) 26 seeds. (e) the output of the proposed MR-based seed finder algorithm. (f) the comparison between the centroids calculated using the CT-based clinical software and the MR-based proposed approach. The arrows in CT images indicate the direction of the CT couch during the scan and on

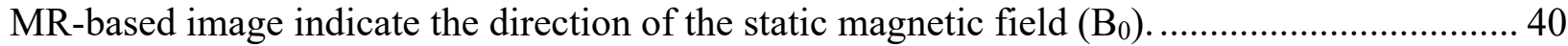

Figure 3.4; The MIP of the calculated QSM using (a) 3T MR images and (b) 1.5T MR images; (c) the comparison between estimated centroids using proposed algorithm based on 1.5T and 3T MR data along with centroids calculated by the clinical CT-based seed finder algorithm. 41

Figure 3.5 (a) dose distribution based on CT-derived positions; (b) dose distribution based on $3 \mathrm{~T}$ MR-derived positions; (c) the absolute difference in dose distributions between CT and MRI calculated as the absolute difference. 42

Figure 4.1; The schematics of the proposed workflow for brachytherapy seed visualization and localization. The steps shown by the "** indicate the proposed post-processing modifications compared to the previously proposed method (Nosrati et al., 2018). 55

Figure 4.2; (a) one sample phantom with 70 implanted seeds; (b) the seed implantation plan (needle loading) which contained four non-spaced seeds shown by the arrows; (c) the Minimum Intensity Projection (MinIP) of the magnitude image from 20 slices at the shortest TE; (d) the Maximum Intensity Projection (MIP) of the phase image from 20 slices at the shortest TE. The full thresholded MIP of the calculated susceptibility maps with the phantom orientated at (e) 0 degree, (f) 45 degrees and (g) 90 degrees with respect to the static magnetic field ( $\left.\mathrm{B}_{0}\right)$; (h) the MIP of the CT image of the same phantom. The arrows in b-g indicate the non-spaced (double-loaded) seeds.

Figure 4.3; (a-d) Comparison between clinical CT-based (MIM Symphony) and the proposed MRbased seed localizer algorithms on each of four phantoms; (e) The Bland-Altman plot for CT and 
MR-derived seed positions for all 321 seeds; (f) The correlation between MR and CT-derived DVH parameters.

Figure 4.4; (a) The estimated distortion map; (b) distortion corrected magnitude image; (c) difference between corrected and uncorrected magnitude images; (d) corrected magnitude image after edge enhancement.

Figure 4.5; (a) For one axial slice of the prostate: (a) CT image; (b) MR magnitude image acquired at $\mathrm{TE}=2.3 \mathrm{~ms}$; (c) MR phase image acquired at $\mathrm{TE}=2.3 \mathrm{~ms}$; (d) estimated prostate and muscle mask for zero referencing during dipole inversion process; (e) the estimated susceptibility map by applying the exact method proposed in(Nosrati et al., 2018); (f) the estimated susceptibility map using the proposed workflow with edge enhancement but without distortion correction and prostate zero referencing; $(\mathrm{g})$ the estimated susceptibility map using the proposed workflow with MEDI +0 but without distortion correction; (h) the estimated susceptibility map using the proposed workflow with edge enhancement, MEDI +0 and distortion correction. 63

Figure 4.6; T2-weighted MR, T1-weighted MR and the MIP reconstruction of a stack of five slices of CT and QSM of three patients. 64

Figure 4.7; CT, QSM and T2-weughted MR images of an axial slice of the prostate which contained a prostatic calcification. The arrows indicate the location of the calcification. 65

Figure 4.8; Comparison between the proposed MR and CT-derived positions in (a) patient with 78 implanted seeds and (b) patient with 66 implanted seeds; (c) Blant-Altman plots of MR-derived vs. CT-derived seed centroids; results; (d) the estimated DVH curves based on CT/MR-derived seed positions for the patient with 78 seeds. 66

Figure 4.9; Schematics of the proposed MRI-only workflow for clinical implementation 68

Figure 5.1; MRI post-processing pipeline 79

Figure 5.2.; U-Net model architecture; blue boxes represent multi-channel feature maps. The size of the input and output labeled images were 256x256. The number of the channels in each layer are shown by a number on top of each box. 81

Figure 5.3; axial T2-wighted MR, and MIP reconstruction (5 mid-slices) of reconstructed QSM and $\mathrm{CT}$ images of 5 patients. 84

Figure 5.4; Example (a) sagittal, (b) axial and (c) coronal slices of the reconstructed QSM for one patient. The arrow in (a) shows an example of non-spaced seeds (double loading). 84

Figure 5.5; Some example automated seed segmentation results on QSM using the trained U-Net model. The top left QSM image shows high susceptibility area in the rectum due to gas (with similar susceptibility values to that of seeds) and the trained model successful .... 86

Figure 5.6; Seed localization and dosimetric analysis in one example patient. (a) 3D representation of CT and MR-derived seeds centroids; (b) the Bland-Altman plot for seed detection differences in $\mathrm{X}, \mathrm{Y}$ and $\mathrm{Z}$ directions with a small bias of -0.003 and $95 \%$ limits of agreement of $[-1.2+1.2] \mathrm{mm}$; (c) axial, sagittal and coronal CT images with identified seed centroids and isodose lines calculated based on CT and (d) axial, sagittal and coronal CT images with identified seed centroids and isodose lines calculated based on MRI (QSM). Prostate contouring was performed on T2-weighted MRI following CT-MRI fusion and the same set of contours were used for dosimetry. 87

Figure 6.1; the MR image post-processing pipeline. 98 
Figure 6.2; (a) internal configuration of the seeds that were studied [41,42]; (b) constructed agarbased phantom containing 5 seeds and 3 calcifications (bone); (c) MR magnitude image at shortest TE; (d) MR phase image at shortest TE; (e) An axial slice of the reconstructed QSM which contains seeds and calcifications; (f) the axial slice of CT that contains seeds and calcifications. 101

Figure 6.3; (a) structure of the studied gold marker ("CyberMark ${ }^{\mathrm{TM}}$ Fiducial Markers," n.d.); (b) constructed agar-based phantom containing 3 gold makers; (c) MR magnitude image at shortest TE; (d) MR phase image at shortest TE; (e) QSM; (f) CT image 102

Figure 6.4; (a) structure of the carbon-coated zirconium marker (BiomarC); (b) prostate phantom containing 3 carbon makers; (c) MR magnitude image at shortest TE; (d) MR phase image at shortest TE; (e) reconstructed susceptibility map (QSM); (f) CT image. The arrows in (c) and (d) indicate an air bubble that was left in the phantom along the needle track during implantation however, it did not affect the QSM process. 103

Figure 6.5; (a) Plastic needle, CT-marker and ProGuide obturators that were studied; (b) constructed agar phantom with 5 inserts including 2 plastic needles with CT-marker obturators, 2 plastic needles with Pro-Guide obturators and one pure titanium rod representing a biopsy needle; (c) meat phantom with 2 hollow plastic needles, one needle with CT-marker and one needle with Pro-Guide obturator; (d) MIP reconstruction of the QSM for the agar-based phantom; (e) MIP reconstruction of the QSM of the meat phantom; (f) MIP reconstruction of the CT of the meat phantom 105

Figure 6.6; The comparison between the QSM results using MEDI and MEDI +0 in (a) meat phantom with two hollow plastic needles (1 and 4) and Pro-Guide obturator (2) and CT-marker (3); (b) prostate phantom with three implanted carbon fiducial markers; (c) agar-based phantom containing three. 107 


\title{
LIST OF ABBREVIATIONS
}

\author{
BT \\ Brachytherapy \\ BW \\ Bandwidth \\ $\mathrm{CNN}$ \\ Convolutional Neural Network \\ CT \\ Computed Tomography \\ DBSCAN \\ Density Based Clustering Application with Noise \\ EBRT \\ External Beam Radiation Therapy \\ FA \\ Flip Angle \\ FOV \\ Field of View \\ HARPERELLA \\ HARmonic PhasE REmovaL using LAplacian operator \\ HDR \\ High Dose Rate \\ LBV \\ Laplacian Boundary Value \\ LDR \\ Low Dose Rate \\ MEDI \\ Morphology Enabled Dipole Inversion \\ MRI \\ Magnetic Resonance Imaging \\ PDF \\ Projection onto Dipole Field \\ QSM \\ Quantitative Susceptibility Mapping \\ REVSHARP \\ Regularization Enabled Varying Sphere Sophisticated Harmonic Artefact \\ reduction of Phase \\ ROI \\ Region Of Interest \\ RT \\ Radiation Therapy \\ SNR \\ Signal to Noise Ratio \\ T1W \\ T1 Weighted \\ $\mathrm{T} 2 \mathrm{~W}$ \\ T2 Weighted \\ TE \\ Echo Time \\ TR \\ Repetition Time \\ TRUS \\ Trans-Rectal Ultrasound \\ UTE \\ Ultra-Short Echo \\ VSHARP \\ Varying sphere Sophisticated Harmonic Artefact reduction of Phase
}




\section{CHAPTER 1}

\section{INTRODUCTION}

Permanent implantation of low-dose-rate (LDR) brachytherapy seeds is a well-established treatment modality for patients with localized prostate cancer. The American Brachytherapy Society (ABS) and the Groupe Europeen de Curietherapie-European Society for Therapeutic Radiology and Oncology (GEC-ESTRO) recommends evaluating the quality of the implant and the dose distribution within 60 days of implantation using a standard computed tomography (CT) (Ash et al., 2000; Davis et al., 2012). Presence of radiographic markers (e.g., gold or silver) within the seed capsule generates excellent positive contrast for seed depiction on CT images, however, due to the relatively poor soft tissue contrast in $\mathrm{CT}$, anatomical delineation is very challenging and significant inter-observer, and intra-observer variabilities have been reported in the CT-based approach (De Brabandere et al., 2013; Dubois, Prestidge, Hotchkiss, Prete, \& Bice, 1998; W. R. Lee, Roach, Michalski, Moran, \& Beyer, 2002). In addition, the contoured prostate volume has been shown to be significantly different between MR and CT images(Dubois et al., 1998).

It is widely accepted that magnetic resonance imaging (MRI) is a better imaging modality for delineation of the prostate and the nearby organs at risk (OARs), however, due to the lack of MR signal from the seeds, they appear as dark voids on conventional MR images which are indifferentiable from other such voids associated with calcifications, cavities, and blood vessels. Thus, CT and MRI are complementary modalities to provide both clear seed identification as well 
as high contrast anatomical visualization of the prostate. Clinical workflows employing CT-MRI fusion taking advantage of both imaging modalities are currently implemented at many centers, however, this requires additional resources and patient transfers. Furthermore, the inherent uncertainties associated with CT-MRI image registration can be significant(Dehghan et al., 2016; Kunogi et al., 2015; Polo et al., 2004b). An MRI-only workflow for post-implant dosimetry of LDR brachytherapy seeds thus would be an ideal solution.

There are two fundamental challenges with implanted brachytherapy seeds in MRI. First, the seeds do not have any internal hydrogen atoms; therefore, do not undergo resonance hence will not produce NMR signal and as a result, they will appear as hypointense regions on conventional MR images. Second, the magnetic susceptibility of the seeds (i.e. titanium encapsulation being a paramagnetic material) is significantly higher than that of the surrounding soft tissue (i.e. diamagnetic material). The susceptibility mismatch induces local magnetic field inhomogeneity in their vicinity that cannot be predicted by the frequency encoding gradient. The local field inhomogeneity, in turn, leads to misplacement of the detected signals resulting in signal loss or signal pileup around the seeds (Hargreaves et al., 2011).

Although several MRI-only approaches have been proposed for depiction and localization on conventional brachytherapy seeds, none of them have demonstrated robust performance translatable to the clinic. An efficient MRI-only brachytherapy LDR post-implant dosimetry workflow requires a robust seed visualization as well as localization performance.

In this thesis, the distinctive magnetic susceptibility of the brachytherapy seeds was used for positive contrast seed visualization. To do so, an optimal Quantitative Susceptibility Mapping (QSM) algorithm was developed and validated on phantoms and prostate patients. In the next step, a seed finder algorithm was developed to evaluate the spatial accuracy of the QSM as well as the 
dosimetric outcome of the proposed MRI-based workflow in comparison with the standard CTbased or CT-MRI fusion-based approaches.

\subsection{Summary of Contributions}

This thesis aimed at developing a robust MRI-based workflow for post-implant dosimetry of permanent seed brachytherapy in prostate patients. This goal was achieved in two main steps: positive contrast seed visualization and automated seed identification.

For seed visualization the feasibility of different MR-contrast mechanisms such that they are differentiable from other signal voids (such as prostatic calcifications and blood vessels) were investigated. Among all investigated approaches, Quantitative Susceptibility Mapping (QSM) generated the most promising results. In general, majority of the previous and current clinical research/applications of QSM are focused on brain imaging for detection of iron overload, hemorrhage, and cerebral demyelination. There are very few studies that used QSM for detection of calcifications in the brain, breast, and prostate. To the best of our knowledge this thesis has been the first study on application of QSM for detection of titanium seeds in prostate cancer patients. The conventional QSM algorithm is mainly designed for brain with a limited range of susceptibility gradients of biological tissues however, in the case of titanium seed implants the susceptibility mismatch between diamagnetic biological tissues and paramagnetic seeds is several orders of magnitude greater than that in brain applications. The steep susceptibility gradient between the seeds and surrounding soft tissues strongly accelerates the spin dephasing near the seeds resulting in severe signal loss (low SNR) around the seeds this effect is significantly weaker when biological substances are the source of susceptibility gradients. In addition, unlike tissue 
susceptibility sources, the seed-induced local field inhomogeneities are strong enough to cause signal mis-mapping and image distortion which needs to be taken into account. Last but not least, none of the previous works in clinical applications of QSM required accurate localization of the susceptibility source but rather aimed at visualization of the susceptibility sources for diagnostic purposes. In this thesis in addition to visualization of the susceptibility sources, the shape of the reconstructed seeds and the spatial accuracy of the QSM was investigated and validated with CT.

To address the above mentioned challenges, in this thesis several changes to the general QSM pipeline were proposed including: optimization of the pulse sequence parameters, distortion correction, local field map estimation, and dipole inversion and an efficient QSM pipeline was developed. To assess of the spatial accuracy of the QSM, which is critical for radiation dosimetry purpose, a robust brachytherapy seed segmentation and 3-dimensional (3D) localization algorithm was developed and compared to the CT-based clinical software. Also, several potential applications of the proposed post-processing pipeline in MR-guided radiation therapy were investigated.

The research findings of this thesis are presented in the form of four peer-reviewed journal papers describing the following topics:

1- Automated brachytherapy seed detection in phantoms using QSM and unsupervised machine learning algorithms: In this study, we developed a QSMbased pipeline to depict brachytherapy seeds with positive contrast in the presence of calcifications. We also developed a seed finder algorithm using unsupervised machine learning. This work was done on a simple agar phantom with 20 stranded 
brachytherapy seeds and a few small pieces of bone representing prostatic calcifications.

2- Clinical feasibility of an MRI-only workflow for post-implant dosimetry of LDR brachytherapy. In this study, we evaluated the initially developed workflow on clinically relevant seed configurations in three patients. The MR sequence parameters and post-processing pipeline were modified for the clinical translation of the technique.

3- Comparison of an MRI-only post-planning workflow with MRI-CT fusion based approach. In this work, we assessed our proposed workflow in 25 patients and compared the seed finder and dosimetric results with the standard CT-MRI fusionbased outcomes. We improved the seed localization algorithm for robust performance in more complex situations.

4- Potential applications of QSM in MR-guided radiation therapy. In this work, we studied other applications of QSM in the detection of other temporary or permanent metallic implants that are used in radiation therapy. We studied different types of fiducial markers, HDR catheters, interstitial brachytherapy CT-marker and obturators and biopsy needles. 


\subsection{Contributions of Authors}

The contribution of the authors of each manuscript incorporated in this dissertation (mentioned in previous section) were as following:

I. Manuscript \#1 (chapter 3) Nosrati R, Soliman A, Safigholi H, Hashemi M, Wronski M, Morton G, Pejović-Milić A, Stanisz G, Song WY. "MRI-based automated detection of implanted low dose rate (LDR) brachytherapy seeds using quantitative susceptibility mapping (QSM) and unsupervised machine learning (ML)". Radiotherapy and Oncology. 2018 Sep 19;129(3):540-7.

R. Nosrati designed and carried out the experiments, designed and performed the post-processing algorithm and drafted the manuscript. A. Soliman helped in phantom construction and adjustments of the MR sequence parameters. H. Safigholi conducted the Monte Carlo simulations for dosimetric analysis. M. Hashemi provided advice regarding the seed localization algorithm. M. Wronski helped with CT scans and critically reviewed the manuscript. G. Morton provided advice throughout the course of this work and assessed the manuscript critically. G. Stanisz assessed the manuscript critically and provided critical feedback. A pejovic-Milic and W. Song partially financed this project and provided advice throughout the project as well as critical feedback to the content, experimental design and the manuscript.

II. Manuscript \#2 (chapter 4): Nosrati R, Song WY, Wronski M, Pejović-Milić A, Morton G, Stanisz G. "Feasibility of an MRI-Only workflow for post-implant dosimetry of low-dose-rate (LDR) prostate brachytherapy: transition from phantoms to patients". Brachytherapy 2019 June pii S1538-4721(19)30124-2. 
R. Nosrati designed and carried out the experiments, designed and performed the post-processing algorithm and drafted the manuscript. M. Wronski helped with CT scans and critically reviewed the manuscript. G. Morton generated brachytherapy plans for phantoms and implanted the prostate phantoms with dummy seeds, referred patients for clinical evaluation, provided advice throughout the course of this work and assessed the manuscript critically. A. Pejovic-Milic assessed the manuscript critically and provided critical feedback. G. Stanisz financed this project and provided advice throughout the project as well as critical feedback to the content, experimental design and the manuscript.

III. Manuscript \#3 (chapter 5): Nosrati R, Wronski M, Tseng C, Chung H, Pejović-Milić A, Morton G, Stanisz G. "Post-implant dosimetry of permanent prostate brachytherapy: comparison of QSM-based MRI-only and CT-MRI fusion workflows". International Journal of Radiation Oncology, Biology and Physics, Under second review; Submision ID: ROB-D-19-01030 (2019). R. Nosrati designed and carried out the experiments, designed and performed the post-processing algorithm and drafted the manuscript. M. Wronski helped with CT scans for validation of the MRderived parameters and critically reviewed the manuscript. G. Morton, C. Tseng and H. Chung referred their patients for clinical evaluation, provided advice throughout the course of this work and assessed the manuscript critically. A. Pejovic-Milic assessed the manuscript critically and provided critical feedback. G. Stanisz financed this project and provided advice throughout the project as well as critical feedback to the content, experimental design and the manuscript. 
IV. Manuscript \#4 (chapter 6): Nosrati R, Paudel M, Ravi A, Pejović-Milić A, Morton G, Stanisz G. "Potential applications of the Quantitative Susceptibility Mapping (QSM) in MR-Guided Radiation Therapy”. Journal Physics in Medicine and Biology. 2019 Jul 16;64(14):145013.

R. Nosrati designed and carried out the experiments, designed and performed the post-processing algorithm and drafted the manuscript. M. Paudel helped with CT scans for validation of the MRderived parameters and critically reviewed the manuscript. G. Morton, provided advice throughout the course of this work and assessed the manuscript critically. A. Pejovic-Milic and A. Ravi assessed the manuscript critically and provided critical feedback. G. Stanisz financed this project and provided advice throughout the project and reviewed the manuscript critically. 


\section{CHAPTER 2}

\section{BACKGROUND}

\subsection{Low Dose Rate Brachytherapy}

Prostate cancer is the most common type of cancer diagnosed in men (Greenlee, HillHarmon, Murray, \& Thun, 2001). Unlike many other cancers, the prostate tumor does not respond to chemotherapy alone. Prostate cancer is usually treated by a combination of therapeutic techniques such as surgery, chemotherapy, hormone therapy and radiation therapy(Hoskin, 2013). The selection of the optimal treatment modality in terms of both safety and efficacy is mainly based on the clinical stage of the disease. According to the cancer staging manual (Edge S.B., Byrd D.R., Compton C.C., Fritz A.G., Greene F.L., 2010): Stage I (T1) tumors are clinically unapparent, Stage II (T2) tumors are confined within the prostate gland, Stage III (T3) tumors extend beyond the prostatic capsule and may invade the seminal vesicle, and Stage IV (T4) tumors are fixed to or invade adjacent organs other than seminal vesicles (e.g. bladder, rectum, pelvic wall)(Boonsirikamchai et al., 2013; Edge S.B., Byrd D.R., Compton C.C., Fritz A.G., Greene F.L., 2010). In prostate radiation therapy, the prescribed dose is delivered by external or internal (brachytherapy) radiation source. Permanent seed brachytherapy, also known as low-dose-rate (LDR) brachytherapy, has been considered as the "gold standard" treatment for low-risk prostate cancer patients(Skowronek, 2013). It provides excellent disease control as well as acceptable normal tissue toxicity. LDR brachytherapy involves the permanent implantation of radioactive sources (seeds) within the prostate gland. 
The use of brachytherapy for prostate cancer was first reported in 1911 only 12 years after the discovery of Radium by Marie and Pierre Curie at that time Radium was administered through urethral catheter(GARZOTTO \& FAIR, 2000; Zaorsky et al., 2017). Currently localized tumors are usually treated with brachytherapy as monotherapy in which several radioactive sources are implanted temporarily utilizing high-dose-rate sources ( $>12 \mathrm{~Gy} / \mathrm{h}, \mathrm{HDR})$ or permanently employing low-dose-rate sources ( 0.4-2 Gy/h, LDR)(Suntharalingam, Podgorsak, \& TÖLLI, 2005) within the prostate, and the whole gland is considered as the target volume.

LDR brachytherapy is delivered through several (between 50-120) LDR radioactive sources encapsulated in cylindrical titanium tube (known as brachytherapy seeds) sealed at both ends with laser-welded titanium end cups(Venselaar Jack, Meigooni Ali S., Baltas Dimos, 2012). Generally, as shown in Fig. 2-1, all seeds are cylinders $4.5 \mathrm{~mm}$ long, $0.8 \mathrm{~mm}$ in diameter with $0.05 \mathrm{~mm}$ wall thickness(Venselaar Jack, Meigooni Ali S., Baltas Dimos, 2012). All LDR seeds contain a radiological marker (a high $\mathrm{Z}$ material for enhancing photoelectric cross-section, typically silver or lead). Titanium encapsulation serves to absorb the emitted low energy photons and electrons(Venselaar Jack, Meigooni Ali S., Baltas Dimos, 2012). The most commonly used lowenergy photon emitting brachytherapy radionuclides are: ${ }^{125} \mathrm{I}$ in the form of silver iodide deposited on the surface of a silver rod (average energy $~ 28 \mathrm{keV}$; half-life: 59.4 days) and ${ }^{103} \mathrm{Pd}$ plated onto
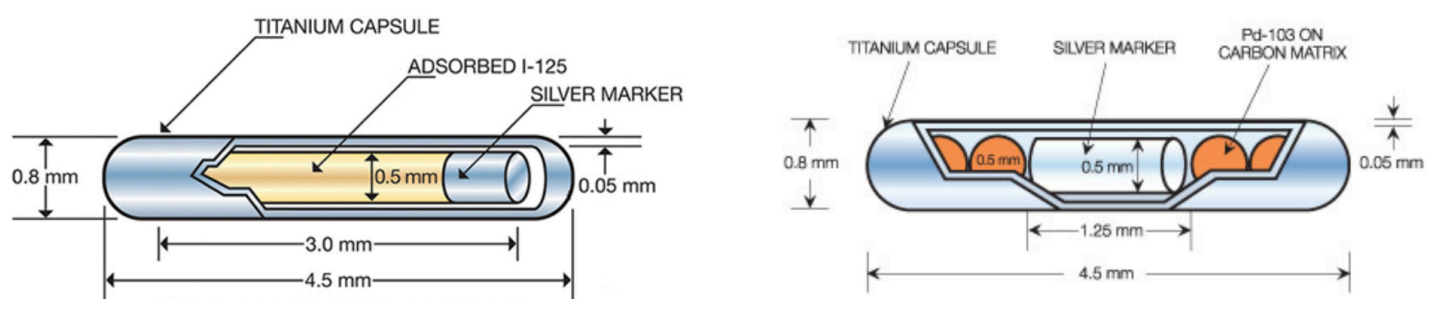

Figure 2.1; LDR brachytherapy seed sample configuration for I-125 and Pd-103 radionuclides 
two graphite pellets on either side of a lead radiographic marker (Yu et al., 1999) (average energy $\sim 21 \mathrm{keV}$; half-life: 17 days)(Suntharalingam et al., 2005).

In LDR brachytherapy, seeds are implanted according to the treatment planning based on either transrectal ultrasound (TRUS) or CT/MR images. According to the plan, the preloaded needles are inserted into the prostate through a template positioned in a way that its center is aligned with the urethra (so-called D line); for better visualization of the urethra in TRUS images, ultrasound micro-bubble contrast agents are employed(Hoskin, 2013).

Brachytherapy is only considered in T1 and T2 stages as monotherapy. HDR brachytherapy is usually performed in intermediate- and high-risk patients for dose escalation as a boost along with external beam radiation therapy (EBRT) whereas LDR brachytherapy is often performed as monotherapy (as the only radiation therapy modality) for low-risk patients(Hoskin, 2013). One of the most effective treatment options for localized low-risk prostate tumors is prostate LDR brachytherapy resulting in minimal toxicity in the healthy tissues(P. Y. Chen, 2009; T. Lim, n.d.; Yu et al., 1999) and comparable or better medium- and long-term outcomes than HDR or EBRT(Kishan \& Kupelian, 2015; Yongjin Wang, Sankreacha, Al-Hebshi, Loblaw, \& Morton, 2006); in addition, non-fractionated schedule of LDR brachytherapy results in minimal workload per patient(Yongin Wang et al., 2006). The outcomes of both LDR and HDR brachytherapy are superior to EBRT with less healthy tissue toxicity. The dose falloff in both LDR and HDR is very steep and less than $10 \%$ of the prescribed dose is delivered to the tissues $>4 \mathrm{~cm}$ from the source(Zaorsky et al., 2017).

Post-implant dosimetry is the gold standard to assess the quality of the treatment, which is typically performed within 30 following the implantation(Orio et al., 2008; Stock \& Stone, 2002). 
Post-implant dosimetry allows possible treatment modifications if dosimetric evaluations reveal that target coverage is inadequate (e.g. by implantation of additional seeds or EBRT); it also provides potential information on the general quality of the implant, disease control, and expected toxicity to the organ at risk (OAR)(Martin, Pugh, et al., 2017). Post-implant dosimetry requires accurate target and organ at risk delineation as well as accurate seed localization; any uncertainties in brachytherapy seed localization or prostate/organ at risk delineation reduces the accuracy hence the quality of the process.

Currently, according to the American Society for Radiation Oncology (ASTRO) and the European Society for Radiotherapy and Oncology (GEC-ESTRO), the CT-based dosimetry is the standard imaging method(Mitina, Christie, Hill, Middlebrook, \& Nadezhdin, 2016; Rosenthal et al., 2011; Salembier et al., 2007) for prostate post-implant dosimetry. Although seeds are clearly visible on $\mathrm{CT}$ (positive contrast), target and normal tissue delineations are challenging due to the poor soft tissue contrast resulting in significant inter- and intra-observer discrepancies in contouring the prostate and organs at risk (OARs)(Polo et al., 2004b; Prete, Prestidge, Bice, Dubois, \& Hotchkiss, 1998; Salembier et al., 2007). It has been shown that CT-based dosimetry can lead to upwards of $33 \%$ variability in D90 (dose delivered to $90 \%$ of the target volume) values(Gregory, Pattison, \& Bibbo, 2015) and V100 (volume that is receiving $100 \%$ of the prescribed dose) has been reported to be extremely sensitive to prostate contouring errors and uncertainties(Mashouf et al., 2016). Recently magnetic resonance imaging (MRI) has been introduced to the LDR post-implant dosimetry workflow to benefit from its superior soft-tissue contrast(Ash et al., 2000; Davis et al., 2012; Segedin \& Petric, 2016). However, due to the lack of MR signal from the seeds, they appear as dark voids on MR images, and seed localization still relies on CT. Besides, in the prostate, on T2-weighted MR images, prostatic calcifications, which 
are a common manifestation(Suh et al., 2008), as well as normal body cavities and blood vessels, often have similar appearances as the seeds (i.e., appear as dark signal voids) making them visually challenging to differentiate. The CT-MRI fusion workflow has been recommended by the recent American Brachytherapy Society (ABS) guideline to take advantage of both imaging modalities by CT-based seed localization with MRI-based target delineation(Davis et al., 2012). Theoretically, the CT-MRI combination allows for both superior soft tissue contrast (afforded by MRI) as well as clear seed depiction (provided by CT)(Polo et al., 2004a). However, in practice, the unavoidable uncertainties associated with the image registration/fusion process lead to nonnegligible dosimetric errors(Tanaka et al., 2006a). It has been shown that the uncertainties associated with the MR-CT fusion may lead to up to $16 \%$ deviation in D90(De Brabandere, Hoskin, Haustermans, Van Den Heuvel, \& Siebert, 2012; Dehghan et al., 2016; Kunogi et al., 2015; Polo et al., 2004b). Various techniques for improving CT-MRI fusion have been proposed(Kunogi et al., 2015; Polo et al., 2004b), but the inherent flaws associated with the process (e.g., patient positioning during image acquisition and organ deformations during transfer) limits these techniques. In addition, the CT-MRI workflow adds extra cost and logistics.

\subsection{Previous Works in MRI-based Post-Implant Dosimetry}

Many efforts have been made to improve the visualization of the seeds on MR images either through minimizing the susceptibility-induced artifacts or creating positive contrast for the seeds.

Seevinck et al. proposed a novel technique for generating positive contrast for small paramagnetic objects such as seeds. Their method is known as center-out RAdial Sampling with Off-resonance Reception (co-RASOR) that employs Ultrashort Echo Time (UTE) pulse sequence and shifts the signal pileup toward the paramagnetic object's center using off-resonance 
reception(De Brabandere, Hoskin, Haustermans, Van Den Heuvel, et al., 2012; H. de Leeuw, Seevinck, \& Bakker, 2013; Hendrik de Leeuw, Moerland, van Vulpen, Seevinck, \& Bakker, 2013b). The co-RASOR method requires prior knowledge of susceptibility values to correctly shift the off-resonance signal to the center of the object. Another developed MR pulse sequence by Stuber et al. employs Inversion Recovery with ON-resonant water suppression (IRON) pulse sequence to illuminate super-paramagnetic material with positive contrast(Stuber et al., 2007). The IRON sequence and a corresponding image processing algorithm for post-implant seed localization has been proposed by Kuo et al. (Kuo, Lee, Tempany, Stuber, \& Prince, 2010a). The co-RASOR and IRON pulse sequences both require significant modifications of the clinical scanners that make the translation process very complicated. In addition, seed visualization in both techniques is firmly orientation dependent.

Application of magnetic susceptibility as a source of contrast in MR imaging has been studied by many research groups for different purposes; especially in brain imaging and neurodegenerative diseases(W. Chen et al., 2014). Susceptibility-based positive contrast or quantitative susceptibility mapping (QSM) for brachytherapy seeds visualization has also been studied, but the proposed methods rely on non-clinical MR pulse sequences or scanners; moreover, the quality of the reconstructed seed images using these methods is significantly worse than that on CT and seed visualization is orientation-dependent (Dong, Chang, Xie, Whitehead, \& Ji, 2015; Shi et al., 2017). Dong et al. have proposed a new algorithm using a fast spin-echo (FSE) pulse sequence with UTE and generated susceptibility-based positive contrast for positive contrast seed depiction(Dong et al., 2015). This study has been performed on very high field strength (4.7T) on phantoms containing few dummy seeds (orientated along the main magnetic field) as well as a toothpick and plastic stick to mimic a natural void and capillary respectively, however, in reality, 
the situation is much more complex as the number of seeds is $60-100$ which are randomly oriented, and there are different structures such as calcification and hemorrhage that exhibit similar appearance in MR images.

There are very limited clinical studies evaluating the feasibility of MRI-based brachytherapy seed detection and localization. Application of contrast-enhanced T1-weighted MR sequences in seed identification has been studied in(Buch et al., 2014; Ohashi et al., 2012a; Tanaka et al., 2006b) however, they have been limited by inconsistent MR sequence parameters, unreliable performance for extraprostatic or non-spaced seed identification. Zijlstra et al. have investigated the feasibility of an MRI-based workflow in which the seed-induced magnetic field distortions (for a specific type of seed) were simulated at different orientations and were used for template matching in realistic cases; although the proposed method has acceptable performance in detection of spaced seeds (average error of $0.8 \pm 0.4 \mathrm{~mm}$ ), by average $33 \%$ of the non-spaced seeds were not identified due to complexity of the field distortion around those seeds(Zijlstra et al., 2017). In addition, the radiation oncologists prefer to clearly observe the seeds for verification of the post-planning seed finder algorithm, but the proposed method is not able to portray the seeds with positive contrast.

Frank et al. have introduced an MR contrast agent (marker) with cobalt-dichloride-N-acetyl cysteine (C4) that is used as the spacer between seeds on stranded brachytherapy seeds which requires structural modifications of the seeds(Frank et al., 2008; T. Y. Lim, Stafford, Kudchadker, Sankaranarayanapillai, Ibbott, Rao, Martirosyan, \& Frank, 2014a). Martin et al. compared the MRI-only LDR post-implant dosimetry (using the stranded seeds with C4 MR-markers as spacers) with the standard MR-CT fusion-based approach(T. Y. Lim, Stafford, Kudchadker, Sankaranarayanapillai, Ibbott, Rao, Martirosyan, Frank, et al., 2014; Martin, Pugh, et al., 2017). 
They have demonstrated that MRI-only dosimetry using C4 MR markers is feasible and accurate. However, there are some limitations associated with $\mathrm{C} 4$ application such as higher cost compared with conventional seeds, the necessity of endorectal coil for marker visualization, and nonapplicable to the plans with non-spaced or loose seeds(Martin, Pugh, et al., 2017). In addition, in this approach, the spacers between seeds are depicted with positive contrast, not the seeds; therefore, seed localization is challenging. It should be considered that in all the approaches mentioned above the implanted seeds appear as dark voids which may be inconvenient for the physicist or the radiation oncologist who review and approve the post-planning after running the automated seed finder and dose calculations.

\subsection{Quantitative Susceptibility Mapping}

Magnetic susceptibility is a dimensionless proportionality constant the indicates the degree of magnetization in the material in an external magnetic field. Diamagnetic substances are those with no unpaired electrons, thus repel the external magnetic field and have negative magnetic susceptibility and paramagnetic substances are those with some unpaired electrons thus are magnetized and have positive magnetic susceptibility. Most of the biological tissues are weakly diamagnetic.

Calculation of the magnetic susceptibility is an inverse problem that is solved using MR phase images obtained by a gradient recalled echo (GRE) MR sequence. The relative local magnetic field $b(r)$ (which can be derived from phase data), is the convolution of the unit dipole kernel $d(r)$ and the magnetic susceptibility at that position $\chi(r)$ (de Rochefort, 2010; Jackson, 1999; T. Liu et al., 2013); in Fourier domain the convolution becomes multiplication as follows: 


$$
b(r)=d(r) \otimes \chi(r) \stackrel{\text { Fourier Transform }}{\longrightarrow} B(k)=D(k) \times \chi(k)
$$

Dipole kernel is the z-component (parallel to $B_{0}$ ) of the magnetic field generated by a single dipole at the origin with Lorentz sphere correction and can be calculated as following(Jackson, 1999; J. Liu et al., 2012a; T. Liu, Liu, et al., 2011):

$$
d(r)=\frac{3 \cos ^{2} \theta_{r}-1}{4 \pi r^{3}} \stackrel{\text { Fourier Transform }}{\longrightarrow} D(K)=\frac{1}{3}-\frac{k_{Z}^{2}}{k^{2}}
$$

The relative local magnetic field $b(r)$ is the local off-resonance frequency map $(f)$ normalized to the gyromagnetic ratio $(\gamma)($ T. Liu et al., 2013). The total field at each voxel is determined from the unwrapped MR phase data. The local field map is determined by the calculation and subtraction of the background field from the total field map. Theoretically susceptibility can be calculated by dividing the local field map by the dipole kernel in a process known as dipole inversion in Fourier domain, However, according to equation II, the dipole kernel is zero on two cone surfaces at the magic angle $\left(\theta \approx 54.74^{\circ}\right)$ therefore, the dipole inversion problem is ill-posed and requires regularization.

In general, QSM is performed in three main steps:

1. Phase unwrapping; since the dynamic range of the phase data are limited to 
$[-\pi, \pi]$ at phase discontinuities $n \times 2 \pi$ need to be added to the phase data to estimate the true field map. Different methods exist for phase unwrapping, which are mainly based on the field continuity assumption.

2. Background field removal; to calculate the local field map induced only by the susceptibility sources within the ROI different techniques have been proposed to calculate the background field which are either based on harmonic properties of the background field(D. Zhou, Liu, Spincemaille, \& Wang, 2014) or based on the projection theorem in Hilbert space(T. Liu, Khalidov, et al., 2011). The most efficient techniques for background field estimation are: Projection onto Dipole Field (PDF)(T. Liu, Khalidov, et al., 2011), Laplacian Boundary Value (LBV)(D. Zhou et al., 2014), HARmonic PhasE REmovaL using LAplacian operator (HARPERELLA)(Li, Avram, Wu, Xiao, \& Liu, 2014), Varying sphere Sophisticated Harmonic Artefact reduction for Phase data (VSHARP)(Wu, Li, Guidon, \& Liu, 2012) , Regularization Enabled Varying sphere Sophisticated Harmonic Artefact reduction for Phase data (REVSHARP)(Kan et al., 2016).

3. Dipole inversion; To solve the inverse ill-posed problem for the magnetic susceptibility, $\chi(r)$, given the local field map, $b(r)$, and dipole kernel, $d(r)$ (described in Equation I) different methods have been proposed. The clinically feasible techniques are Morphology Enabled Dipole Inversion (MEDI) with L1 norm-based iterative regularization algorithm(J. Liu et al., 2012a; T. Liu, Liu, et al., 2011), L2 norm-based regularization algorithm(Bilgic et al., 2014) using iterative Least Squares Root method (iLSQR)(Li, Wu, \& Liu, 2011). 


\subsection{Machine Learning for Medical Image Analysis}

Machine Learning (ML) is an exciting and rapidly growing field of research. It focuses on data analysis technique that teaches computers to perform tasks such as categorization or object detection like the human brain. ML has drawn significant attention to solving problems in the past decades in many areas, including medical image processing and computer vision. ML is performed by two main techniques: Supervised learning and unsupervised learning, which are briefly explained in the following sections.

\subsubsection{Supervised machine learning}

The aim of supervised machine learning is to generate a model that can predict the desired output based on evidence in the presence of uncertainty. Supervised machine learning utilizes a labelled set of data known as training data to train a model that can generate reasonable predictions on the future outputs for a given range of inputs based on classification and regression techniques. Training is an iterative process that aims at minimizing a target cost function by adjusting the parameters (weights) of the model. In supervised learning depending on the application, the optimal model architecture is determined then the parameters (weights) of the model are iteratively learned through the training process. Every labelled instance in the training dataset in supervised learning is represented by a set of features that could be continuous, categorical or binary. The features that best describe the corresponding labels are identified through training and are used for future predictions(Maglogiannis, 2007).

Supervised learning has numerous applications in different fields, including medical image segmentation, which was used in this thesis. For automated medical image segmentation, a series 
of images (CT, MRI, etc.) with the corresponding manual segmentation of the target(s) (such as tumor, OAR, seed) are required to train the model. The larger the number of training dataset the more efficient predictions; however, in practice, the number of available labelled medical images for training is very limited. Thus training data augmentation techniques and the choice of the appropriate network architecture are critical steps in medical image segmentation using supervised ML.

\subsubsection{Unsupervised machine learning}

Unsupervised learning un-cover hidden structures and interpret the data to find subpopulations in large cohorts based on their intrinsic patterns. In this type of ML there is no need

for labelled training data and the algorithm partitions the data based on their similarity. Clustering is the most common unsupervised ML technique that is increasingly used in medical image processing such as tumor delineation and image segmentation, object recognition, gene sequence analysis, etc. (Dao-Qiang Zhang, Song-Can Chen, Zhi-Song Pan, \& Ke-Ren Tan, n.d.; Larrañaga et al., 2006; Manogaran et al., 2017; Nasrabadi, 2007; S. Wang \& Summers, 2012; Yang, Zhan, Xie, Zhao, \& Kurihara, 2017; Zacharaki et al., 2009).

In this thesis, supervised machine learning was used for brachytherapy segmentation on calculated susceptibility maps, and unsupervised learning was used for seed localization. 


\title{
3. MRI-BASED AUTOMATED DETECTION OF IMPLANTED LOW DOSE RATE (LDR) BRACHYTHERAPY SEEDS USING QUANTITATIVE SUSCEPTIBILITY MAPPING (QSM) AND UNSUPERVISED MACHINE LEARNING (ML)
}

\begin{abstract}
Published in the Journal of Radiotherapy and Oncology: Nosrati R, Soliman A, Safigholi H,
Hashemi M, Wronski M, Morton G, Pejović-Milić A, Stanisz G, Song WY. “MRI-based automated detection of implanted low dose rate (LDR) brachytherapy seeds using quantitative susceptibility mapping (QSM) and unsupervised machine learning (ML)". Radiother Oncol.
\end{abstract} 2018 Sep 19;129(3):540-7.

Authors' contributions: R. Nosrati designed and carried out the experiments, designed and performed the post-processing algorithm and drafted the manuscript. A. Soliman helped in phantom construction and adjustments of the MR sequence parameters. H. Safigholi conducted the Monte Carlo simulations for dosimetric analysis. M. Hashemi provided advice regarding the seed localization algorithm. M. Wronski helped with CT scans and critically reviewed the manuscript. G. Morton provided advice throughout the course of this work and assessed the manuscript critically. G. Stanisz assessed the manuscript critically and provided critical feedback. A pejovic-Milic and $W$. Song partially financed this project and provided advice throughout the project as well as critical feedback to the content, experimental design and the manuscript. 


\subsection{Abstract}

Permanent seed brachytherapy is an established treatment option for localized prostate cancer. Currently, post-implant dosimetry is performed on CT images despite challenging target delineation due to limited soft tissue contrast. This work aims to develop an MRI-only workflow for post-implant dosimetry of prostate brachytherapy seeds.

A prostate mimicking phantom containing twenty stranded I-125 seeds and calcifications was constructed. A three-dimensional gradient-echo MR sequence was employed on 3T and 1.5T MR scanners. An optimized quantitative susceptibility mapping (QSM) technique was applied to generate positive contrast for the seeds and calcifications. Seed numbers, centroids, and orientations were determined using unsupervised machine learning algorithms. The geometrical positions and dose distribution were compared to the clinical CT-based approach.

The optimized QSM-based method generated high quality positive contrast for the seeds that was significantly different from that for calcifications and could be easily differentiated by thresholding. The estimated seed centroids from both $3 \mathrm{~T}$ and $1.5 \mathrm{~T}$ MR data were in perfect agreement with the standard CT-based seed detection algorithm (maximum difference of $0.7 \mathrm{~mm}$ ). The estimated seed orientations were highly correlated with the actual orientations $(\mathrm{R}>0.98)$.

The proposed MRI-based workflow enabling an accurate and robust means to localize the seeds (position and orientation) upon validation on complex seed configurations, has the potential to replace the current widely-practiced CT-based workflow. 


\subsection{Introduction}

Prostate cancer is the most common type of cancer diagnosed in men (Cronin et al., 2018; Greenlee et al., 2001; Kirby, 2018). Permanent seed brachytherapy also known as low-dose-rate (LDR) brachytherapy is the standard option for low and intermediate risk prostate cancer (Chin et al., 2017; Skowronek, 2013). LDR brachytherapy involves permanent implantation of radioactive sources (seeds) within the prostate gland. To evaluate the quality of the implant, post implant dosimetry is typically performed at day 30 following the implantation (Orio et al., 2008). The implant quality is mainly assessed through Dose Volume Histogram (DVH)-related parameters which have been reported to be dependent on the imaging modality (Polo et al., 2004b).

Currently, according to the American Society for Radiation Oncology (ASTRO) and the European Society for Radiotherapy and Oncology (GEC-ESTRO), the CT-based dosimetry is the standard method (Mitina et al., 2016; Rosenthal et al., 2011; Salembier et al., 2007) for prostate post-implant dosimetry. Although seeds are clearly visible on CT, target and normal tissue delineations are challenging due to the poor soft tissue contrast resulting in significant inter- and intra-observer discrepancies in contouring the prostate and organs at risk (OARs) (De Brabandere, Hoskin, Haustermans, Van Den Heuvel, et al., 2012; Polo et al., 2004b; Prete et al., 1998; Salembier et al., 2007). It has been shown that CT-based dosimetry can lead to upwards of $33 \%$ variability in $\mathrm{D}_{90}$ values (Gregory et al., 2015) and $\mathrm{V}_{100}$ has been reported to be extremely sensitive to prostate contouring errors and uncertainties (Mashouf et al., 2016). Also, many clinically used CT-based seed finder algorithms require a priori knowledge of the exact number of implanted seeds (which may not necessarily reflect the number of present seeds at the time of post-planning due to possible seed migration and loss) and determine centroids of the seeds thereafter (but not 
orientations). This makes the process vulnerable to cases of seed migration, leading to potential double counting of a single seed or counting nearby calcifications of similar dimensions.

On the other hand, MRI offers the best soft tissue contrast amongst all medical imaging modalities. However, seeds appear as diffused dark voids because of absence of hydrogen atoms that are the origins on MRI signal. In addition, on T2-weighted MR images for prostate imaging, prostatic calcifications, which are commonly found (Suh et al., 2008), as well as normal body cavities, often have similar appearances as seeds (i.e., appear as dark signal voids) on typical MR images making them visually difficult to differentiate. To benefit from the superior soft tissue contrast of MRI for post implant dosimetry, efforts have been made to develop MRI-based workflows as an alternative to CT in which MR signal voids are counted as seeds. However, due to image distortions around the seeds the proposed seed finder algorithms have not been clinically reliable (De Brabandere et al., 2013; R. J. Lee et al., 2007; Tanaka et al., 2006c). Ideally, the CTMRI combination allows for both superior soft tissue contrast as well as accurate seed localization (Polo et al., 2004a). However, uncertainties associated with the image registration/fusion process can lead to non-negligible dosimetric uncertainties (Tanaka et al., 2006a). Various techniques for improving CT-MRI fusion have been proposed (Kunogi et al., 2015; Polo et al., 2004b) but inherent uncertainties associated with the process (e.g., organ deformations during transfer) limits these techniques. In addition, the CT-MRI workflow adds extra cost and time.

There are two main challenges with MR imaging of implanted brachytherapy seeds. First, the seeds do not have any internal hydrogen atoms therefore do not produce the MR signal and as a result will appear as hypointense regions on MR images. Secondly, the magnetic susceptibility of the seeds (i.e., titanium encapsulation being a paramagnetic material) is significantly greater 
than that of the surrounding soft tissue (i.e., diamagnetic material). The resulting magnetic susceptibility gradient induces magnetic field inhomogeneity in their vicinity that cannot be predicted by the frequency encoding gradient. The local field inhomogeneity in turn leads to misplacement of the detected signals resulting in signal loss or pile-up around the seeds(Hargreaves et al., 2011).

In general, to overcome the lack of positive contrast for small paramagnetic objects (e.g., seeds, MR contrast agents, iron depositions, calcifications, etc.) on the MR images, different solutions have been proposed. Encapsulated MR markers have been developed which are placed between seeds on a strand acting as spacers (T. Y. Lim, Stafford, Kudchadker, Sankaranarayanapillai, Ibbott, Rao, Martirosyan, \& Frank, 2014a; T. Y. Lim, Kudchadker, Wang, Bathala, et al., 2016; T. Y. Lim, Kudchadker, Wang, Stafford, et al., 2016). However, the current application of the seeds with marker-spacers is limited because of their significantly higher cost and availability on only stranded seeds and not loose seeds. Other proposed solutions include new MR pulse sequences along with post-processing algorithms. For instance, Inversion Recovery with ON-resonant water suppression (IRON) pulse sequence suppresses the signal from background tissue and produces positive contrast around paramagnetic objects (Stuber et al., 2007); center-out Radial Sampling with Off-resonance Reception (co-RASOR) method, applies radial gradients with specific frequency offsets to produce circular signal pileups around field perturbers (e.g. seeds) and then focuses the signal pileup at the center of the paramagnetic object $(\mathrm{H}$. de Leeuw et al., 2013; Hendrik de Leeuw, Moerland, van Vulpen, Seevinck, \& Bakker, 2013a); this method requires some prior information to find the optimal frequency offset (Dong et al., 2015). These techniques employ non-clinical pulse sequences and their implementation requires significant modification of the scanner hardware/software making the clinical translation difficult. Utilizing 
"magnetic susceptibility" as a source of contrast for paramagnetic seeds has also been investigated. Magnetic susceptibility is an inherent property of a material and reflects the degree of magnetization when placed in an external magnetic field such as the MR main magnetic field. Magnetic susceptibility is not measured directly in MRI and the calculation requires extensive post-processing steps. In previous studies done to achieve the optimal results for the distinctive susceptibility of the seed, ultra-high field strength scanners $(>4.7 \mathrm{~T})$ and non-clinical pulse sequences were applied (Dahnke, Liu, Herzka, Frank, \& Schaeffter, 2008; Dong et al., 2015). In addition, in all mentioned techniques, there is a strong dependence between the perceived positive contrast of the seeds and their orientation.

A robust MRI-only pipeline for post-implant dosimetry requires both, positive contrast seed visualization as well as an efficient seed finder (localization) algorithm; however, most of the proposed MR-based seed visualization algorithms lack a seed localization algorithm and there are very limited previous works on MR-based seed localization. Kuo et al. have proposed a technique based on Laplacian of Gaussian (LoG) to localize the seeds on IRON images however, the resulting mean error was $3 \mathrm{~mm}$ with $2 \%$ seed detection error (Kuo, Lee, Tempany, Stuber, \& Prince, 2010b). Zijlstra et al. have investigated the feasibility of a correlation-based template matching algorithm on simulated seed-induced phase shift on phase images; their proposed method has acceptable performance (average error of $0.8 \pm 0.4 \mathrm{~mm}$ ) for well-spaced seed ( $>5 \mathrm{~mm}$ apart) however, almost half (about $43 \%$ ) of the non-spaced or clumped seeds were incorrectly detected (Zijlstra et al., 2017).

To the best of our knowledge application of machine learning (ML) for post-implant localization of brachytherapy seeds on MR images has not been studied. ML is a data analysis 
technique that teaches computers to perform tasks such as categorization or object detection like human brain. ML has drawn significant attention for solving problems in the past decades in many areas including medical image processing and computer vision. ML employs two main techniques: (1) supervised learning utilizes a training dataset to build a model that can predict future outputs for a given range of inputs based classification and regression techniques and (2) unsupervised learning uncover hidden structures and interpret the data to find subpopulations in large cohorts based on their intrinsic patterns. Clustering is the most common unsupervised ML techniques that is increasingly used in medical image processing such as tumor delineation and image segmentation, object recognition, gene sequence analysis, etc. (Dao-Qiang Zhang et al., n.d.; Larrañaga et al., 2006; Manogaran et al., 2017; Nasrabadi, 2007; S. Wang \& Summers, 2012; Yang et al., 2017; Zacharaki et al., 2009).

In this work, we proposed a susceptibility mapping pipeline that employs clinically available MR pulse sequences (applied on 1.5T and 3T scanners) and generates positive contrast for the seeds and calcification but with significantly different susceptibility values; then, an unsupervised machine learning algorithm was applied for 3D image segmentation and finding centroids and orientations of the seeds. For validation, the results of the optimal MR-based workflw are compared to the conventional CT-based seed finder software (VariSeed ${ }^{\mathrm{TM}}$, Varian, Palo Alto, CA).

\subsection{Materials and Methods}

\subsubsection{Phantom preparation}

A prostate tissue mimicking phantom with similar $T_{1}$ and $T_{2}$ relaxation times to prostate (Hattori et al., 2013) consisted of: $3 \%$ agar, $0.29 \% \mathrm{NaCl}, 0.03 \%$ (by weight) and $22.2 \mu \mathrm{mol} / \mathrm{kg}$ of 
$\mathrm{NaN}_{3}$ (Sigma-Aldrich Canada Co.) was constructed. Each ingredient was sequentially dissolved in distilled water while being heated. To construct a two-layered phantom, half of the prepared mixture was poured into a plastic cylindrical container and cooled to the room temperature. Twenty I-125 dummy seeds ( $4.8 \mathrm{~mm}$ long and $0.8 \mathrm{~mm}$ wide; IsoAid Advantage ${ }^{\mathrm{TM}}$ ) were placed in five strands on top of the first layer with different orientations. Three pieces of calcification (obtained from sheep cortical bone which has similar composition to human calcification (Rehman, Smith, Hench, \& Bonfield, 1995)) were shaped similar to the seeds with different lengths $(2,4.5$, and 8 $\mathrm{mm})$. The calcifications were placed next to seeds; the second portion of the mixture was poured into the container and was slowly cooled at room temperature for about an hour and then was stored in the fridge at $4^{\circ} \mathrm{C}$ overnight. Figure $3.1-\mathrm{a}$ shows the configuration of the seeds and calcifications in the phantom before pouring the second layer.

\subsubsection{Data Acquisition Parameters}

The first set of MR images were acquired on a 3T scanner (Philips Achieva ${ }^{\mathrm{TM}}$ ) using an 8channel head coil array. The phantom was scanned with a 3D multi echo gradient echo sequence (ME-GRE) with the following sequence parameters: $\mathrm{TE}_{1}=2 \mathrm{~ms}$; number of echoes $=4$; echo spacing $=1.5 \mathrm{~ms} ; \mathrm{TR}=30 \mathrm{~ms} ;$ flip angle $=15^{\circ} ; \mathrm{FOV}=90 \times 90 \times 21 \mathrm{~mm}^{3} ;$ resolution $=0.7 \mathrm{~mm}$ isotropic; number-of-averages $=3$. The total scan time was $5 \mathrm{~min}$ and $58 \mathrm{sec}$.

The phantom was also scanned with a $1.5 \mathrm{~T}$ scanner (Philips Ingenia ${ }^{\mathrm{TM}}$ ) using an 8-channel head coil array. ME-GRE sequence with the following parameters was applied: $\mathrm{TE}_{1}=2.5 \mathrm{~ms}$; number of echoes $=4$; echo spacing $=2.1 \mathrm{~ms} ; \mathrm{TR}=30 \mathrm{~ms} ;$ flip angle $=15^{\circ} ; \mathrm{FOV}=90 \times 90 \times 18$

$\mathrm{mm}^{3}$; resolution $=0.7 \mathrm{~mm}$ isotropic; number-of-averages $=4$. The acquisition time was $7 \mathrm{~min}$ and $2 \mathrm{sec}$. 
The CT scan was performed according to an institutionally standard protocol for CT-based prostate permanent seed post-implant assessment with the following parameters: $120 \mathrm{kV}, 325$ $\mathrm{mAs}$, and $3 \mathrm{~mm}$ slice thickness.

\subsubsection{Calculation of 3D Magnetic Susceptibility Maps}

Phase images were used to quantify the susceptibility at each voxel. In brief, assuming $\mathrm{B}_{0}$ is the main magnetic field in the z-direction, the susceptibility-induced field distortions (for a nonconductive susceptibility source) can be derived by solving the Maxwell's equations and the Lorentz's sphere correction (Jenkinson, Wilson, \& Jezzard, 2004). The perturbation solution to the Maxwell's equations in continuous space shows that the relative local magnetic field perturbation $b(r)$ (which can be derived from phase data), is the convolution of the unit dipole kernel $d(r)$ and the magnetic susceptibility at that position $\chi(r)$ (de Rochefort, 2010; Jackson, 1999; T. Liu et al., 2013). In Fourier domain the convolution becomes multiplication as follows:

$$
b(r)=d(r) \otimes \chi(r) \stackrel{\text { Fourier Transform }}{\longrightarrow} B(k)=D(k) \times \chi(k)
$$

where dipole kernel is the z-component (parallel to the main magnetic field, $B_{0}$ ) of the magnetic field generated by a single dipole at the origin which can be calculated at different positions (r) within the field of view as following(Jackson, 1999; J. Liu et al., 2012a; T. Liu, Liu, et al., 2011): 


$$
d(r)=\frac{3 \cos ^{2} \theta_{r}-1}{4 \pi r^{3}} \stackrel{\text { Fourier Transform }}{\longrightarrow} D(K)=\frac{1}{3}-\frac{k_{Z}^{2}}{k^{2}}
$$

The relative difference local field $b(r)$ is the local off-resonance frequency map, $f$ normalized to the gyromagnetic ratio $(\gamma)$ (T. Liu et al., 2013). The total field at each voxel is determined from the unwrapped MR phase data and the relative local field map (also known as relative difference field) is determined by calculation and subtraction of the background field from the total field; The dipole kernel is theoretically calculated at every voxel using equation II. Susceptibility is then estimated by dividing the local field map by the dipole kernel (dipole inversion) in Fourier domain (equation I). However, according to the equation II, the dipole kernel is zero on two cone surfaces at the magic angle $\left(\theta \approx 54.74^{\circ}\right)$ therefore the dipole inversion problem is ill-posed and requires regularization.

In this work, the 3D susceptibility map was calculated using MatLab software (The MathWorks-R2016b) in the following steps:

1. The raw phase data from multi echo GRE sequence were unwrapped temporally over successive echoes using the graph-cut method (Bioucas-Dias \& Valadão, 2007) with magnitude weighting to estimate the total field;

2. The background field was calculated using Projection onto Dipole Field (PDF) method (T. Liu et al., 2013; T. Liu, Khalidov, et al., 2011) then it was subtracted from the total field (from the previous step) to estimate the local field map along the main magnetic field. 
3. The non-linear least square fitting method was employed to calculate the offresonance frequency map, $f$ at location, $r$ using the original magnitude data and background removed phase data (the local field from the previous step) (de Rochefort, Brown, Prince, \& Wang, 2008; Kressler et al., 2010; T. Liu et al., 2013):

$$
f_{r, \theta_{0 r}}=\operatorname{argmin} \sum_{j=1}^{\# \text { echoes }}\left\|M_{r, T E_{j}} e^{i \theta_{r, T E_{j}}}-M_{r, T E_{j}} e^{i\left(f_{r} \times T E_{j}+\theta_{r, 0}\right)}\right\|_{2}^{2}
$$

where $M$ and $\theta$ denote magnitude and background removed phase data respectively and $\theta_{0 r}$ denotes the initial phase.

4. The frequency map was spatially unwrapped using the region growing algorithm(K. Zhou, Zaitsev, \& Bao, 2009).

5. The susceptibility values at each voxel were calculated using a Morphology Enabled Dipole Inversion (MEDI) method with regularized L1 minimization (J. Liu et al., 2012a; T. Liu, Liu, et al., 2011); the complex exponential function was employed in the data fidelity term. The mathematical basis of the method is presented in Liu et al. (T. Liu et al., 2013) but in brief the following unconstrained minimization problem was solved using an iterative approach to quantify the susceptibility at different positions, $\chi_{r}$ :

$$
\chi_{r}=\operatorname{argmin}_{\chi_{r}} \lambda\left\|W\left[e^{i D_{r} \chi_{r}}-e^{i f_{r}}\right]\right\|_{2}^{2}+\left\|G_{M} G_{\chi}\right\|_{1}
$$


where $\lambda$ is the regularization parameter which was optimized for seed depiction; $D_{r}$ is the Fourier domain dipole kernel at position $r ; W$ is a weighting matrix calculated using a binary mask normalized to the noise standard deviation at each voxel to remove the unreliable estimated frequencies. Matrix $W$ was tuned at each iteration using the Model Error Reduction Through Iterative Tuning (MERIT) technique (T. Liu et al., 2013). $G_{M}$ is the gradient of the magnitude image (anatomical/morphological prior); $G_{\chi}$ is the gradient of the estimated susceptibility map; indeed the $\mathrm{L} 1$ regularization term is utilized to use the magnitude image as a guide and ensure that the edges in the estimated susceptibility map correspond to an edge in the magnitude image (J. Liu et al., 2012a; T. Liu, Liu, et al., 2011).

\subsubsection{Seed Localization on 3D QSM}

The calculated susceptibility maps were thresholded, binarized and converted into point cloud data to extract the spatial distribution of the positive contrast voxels. The resulting 3D binary positive contrast images were then analyzed through unsupervised machine learning (ML) algorithms in MatLab. Clustering is the most well-known unsupervised ML algorithm that partitions the data into groups (clusters) based on their similarities. Spatial clustering was applied to the processed MR images to segment the seeds and estimate their centroids based on the spatial distribution of the point cloud data.

The seed identification and localization (centroid and orientation) was performed in the following steps: 
1- The thresholded images were converted into point cloud with 3D spatial information;

2- $\quad$ The optimal number of implanted seeds was calculated using Silhouette criterion (Rousseeuw, 1987) for K-means clustering (Hartigan \& Wong, 1979); the possible range for number of clusters was assumed to be the number of implanted seeds \pm 6 (to account for the probability of seed loss/migration, erroneous placement of additional seeds and presence of calcifications);

3- K-medoid clustering algorithm (Park \& Jun, 2009; Sander, Ester, Kriegel, \& Xu, 1998) was used to find the medoid (centroid) of each cluster (seed). The central data point within each cluster (seed) was found through minimizing the total intra-cluster variance by solving the following minimization problem(Berkhin, 2006):

$$
J=\operatorname{argmin}_{c} \sum_{i=1}^{k} \sum_{x \in C(i)}\left\|x_{n}-\mu_{i}\right\|_{2}^{2}
$$

where $\mu_{i}$ is one of the actual data points that is initially chosen randomly as a possible medoid, $k$ is the number of clusters and $x_{i}$ is the $i$ th data point in $k$ th cluster. The algorithm uses the K-means ${ }^{++}$algorithm for cluster medoid initialization with distance being the squared Euclidean distance; 
4- The orientation of each seed was determined using eigenvector analysis in two steps; finding the gradient of the original 3D QSM and then performing singular value decomposition (SVD) (Henry \& Hofrichter, 1992).

\subsubsection{Result Validation and Statistical Analysis}

The centroids calculated through the MRI-only based proposed method were compared to those calculated with a CT-based commercial seed finder algorithm (VariSeed 8.0, Varian, Palo Alto, CA). After transformation of the coordinate system between CT and MR using MoorePenrose pseudoinverse matrix calculation, the mean differences between $x, y$ and $z$ positions were calculated. The two-tailed paired sample t-test and one-way ANOVA were used to investigate the difference between CT- and MRI-derived geometrical positions. The estimated seed orientations were compared with the known orientations of each strand in the constructed phantom. Statistical analysis was performed in SPSS software (IBM SPSS statistics 24). The statistical significance was assumed to be at or below 0.05 .

\subsubsection{Dosimetric Analysis}

To investigate the dosimetric impact of seed localization using the proposed workflow with the clinical CT-based approach, the 3D dose distribution for both seed positions were calculated using the Monte Carlo N-Particle (MCNP) simulations for a low energy I-125 (IsoAid Advantage, model IAI, 125A) brachytherapy seed with $0.1 \mathrm{~mm}$ resolution. First, MC simulations was used to benchmark the TG-43(Rivard et al., 2004) dosimetric parameters such as radial dose and 2D anisotropy functions in a water phantom, and the results were compared and validated against previously published data(Rivard et al., 2004). 
Second, the 3D MC dose distribution (Mev/g/decay) of a single seed was converted into $\mathrm{Gy} / \mathrm{U}$ assuming $1 \mathrm{mCi}=1.27 \mathrm{U}$, and $\mathrm{T}_{1 / 2}=59.6$ days for I-125 LDR seeds (Levitt, Purdy, Perez, \& Poortmans, 2012). Further details of factor conversion process are provided in references (Safigholi, Sardari, Karimi Jashni, Mahdavi, \& Meigooni, 2013; Zhang, Baker, McKinsey, \& Meigooni, 2005). Finally, the estimated 3D matrix of dose distribution with $0.1 \mathrm{~mm}^{3}$ resolution was translated and rotated according to the estimated positions (centroids and orientations) of each seed and the overall dose distribution was calculated by superposition. The absolute difference $(\mathrm{Gy} / \mathrm{U})$ of dose distributions was calculated $\left(\operatorname{Dose}_{(\mathrm{MR})}-\right.$ Dose $\left._{(\mathrm{CT})}\right)$. The inter-seed attenuation (Safigholi et al., 2013) and phantom heterogeneities (calcifications) were not taken into account in dose calculations.

\subsection{Results}

Generating Positive Contrast for the Seeds Using QSM

Figure 3.1 shows the constructed gel phantom, the raw magnitude and phase images (acquired by the proposed GRE sequence using 3T scanner) and the calculated images at different steps of the QSM process. The MR acquisition time with $0.7 \mathrm{~mm}$ isotropic resolution and $90 \mathrm{~mm}$ by $90 \mathrm{~mm}$ FOV (142 cc volume of the phantom) was less than $6 \mathrm{~min}$ in duration and the whole image processing pipeline took $90 \mathrm{~s}$.

Figures 3.1-b and 1-c show the maximum intensity projection (MIP) of the CT images scanned with two different orientations (with $90^{\circ}$ differences). The appearance of seeds on CT images is known to depend on seed orientation because of the $\mathrm{CT}$ metal artifact removal algorithm (OMAR) while as one can see from Figure 3.1-h the proposed QSM algorithm clearly depicts the 
seed orientations irrespective of their orientation in the MR bore. The necessity of phase unwrapping steps (temporal and spatial) is evident in Figure 3.1-e in where phase wrapping at later echo times is obvious.

The susceptibility map (Figure 3.1-h) was calculated using the optimal Lagrangian multiplier, $\lambda$ of 400 . The estimated susceptibility values were significantly different between the seeds and calcifications $(p<0.01)$. It can be seen in Figure 3.1-h that in the thresholded susceptibility map, the calcifications completely disappear and hence the process is robust in differentiating the seeds from calcifications. In fact, the proposed algorithm has successfully nulled the appearance of calcifications in the final output, producing an image with only the seeds in positive contrast.
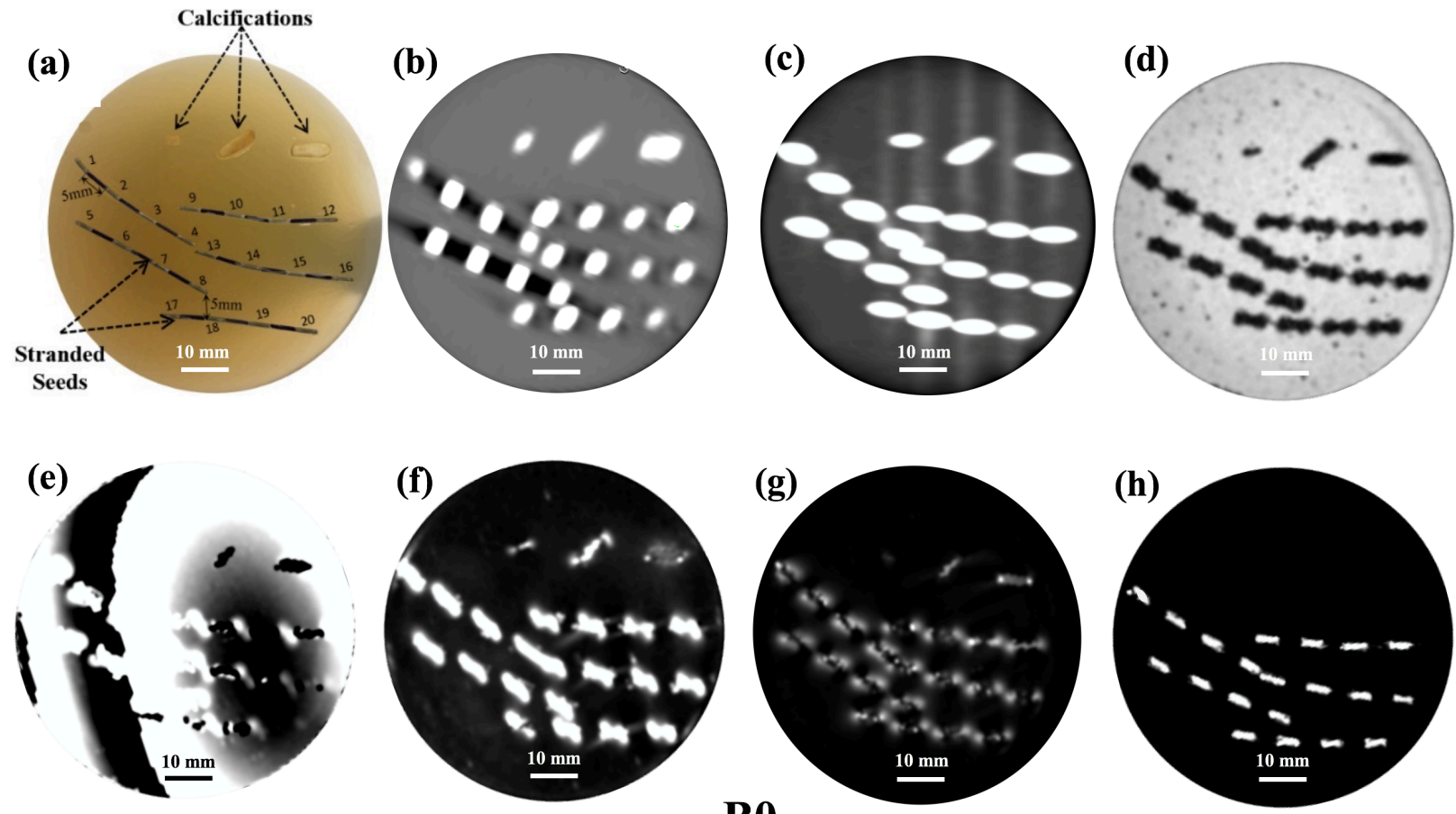
Figure 3.1; (a) The constructed phantom containing 20 seeds (in 5 strands) and three calcifications. MIP reconstruction of the $\mathrm{CT}$ image obtained while the phantom axis (along the $\mathrm{BO}$ arrow) was (b) perpendicular (c) parallel to the CT bed. MIP reconstruction of (d) $3 \mathrm{~T}$ MR magnitude image at $\mathrm{TE}=6.5 \mathrm{~ms}$; (e) 3T MR phase image at $\mathrm{TE}=6.5 \mathrm{~ms}$; (f) phase image after temporal unwrapping and background field removal using PDF method; (g) The estimated off resonance frequency map; (h) the estimated susceptibility map. The BO arrow indicates the direction of the MR static magnetic field.

The minimum distance between the seeds anywhere was $1.5 \mathrm{~mm}$ and occurred between the seeds \#4 and \#13 (Figure 3.1-a). On the thresholded QSM image (Figure 3.1-h), these seeds were successfully differentiated one from one another.

The average length of the seeds in the thresholded QSM was $4.6 \pm 0.3 \mathrm{~mm}$ and the average width of the seeds was $0.9 \pm 0.2 \mathrm{~mm}$ compared with the actual seed dimension of $4.5 \times 0.8 \mathrm{~mm}^{2}$.

\subsubsection{Seeds Localization}

The seed localization process is shown in Figure 3.2. The point cloud was calculated as shown in Figure 3.2-a. The optimal number of clusters, $\mathrm{K}(K \in 20 \pm 6)$ was found to be twenty as shown in Figure 3.2-b having the greatest Silhouette value $(\sim 0.90)$. The estimated medoids (centroids) and orientation of each cluster are shown in Figure 3.2-c. The estimated orientations of the seeds using an eigenvector analysis are in very good agreement with the known orientations shown with dashed lines in Figure 3.2-c $\left(R^{2}>0.95\right)$. The maximum deviation was found to be less than $10^{\circ}$ for only one seed (the seed \#18).

The output of the clinical CT-based algorithm (VariSeed 8.0) along with the comparison between centroids calculated with the CT-based clinical software and the proposed MR-based algorithm are presented in Figure 3.3. The VariSeed seed finder algorithm only finds 
approximately as many seeds as the user inputs; in Figure 3.3-c the number of implanted seeds was defined by the user to be 14 and the software found 18 seeds; in Figure 3.3-d, the number of implanted seeds was defined as 26 by the user and the software detected 26 seeds is the phantom. Although the VariSeed seed finding algorithm treats each seed as a point (ignoring seed orientation) as shown in Figure 3.3-a and b the identified seed orientation is always in the CT scanning direction.

The comparison between CT- and 3T MR-based calculated centroids is demonstrated in Figure 3.3-f. There was no significant difference between the centroids calculated with these two methods $(p<0.01)$; the maximum and mean difference between positions in all three dimensions were $0.7 \mathrm{~mm}$ and $0.3 \mathrm{~mm}$ respectively.
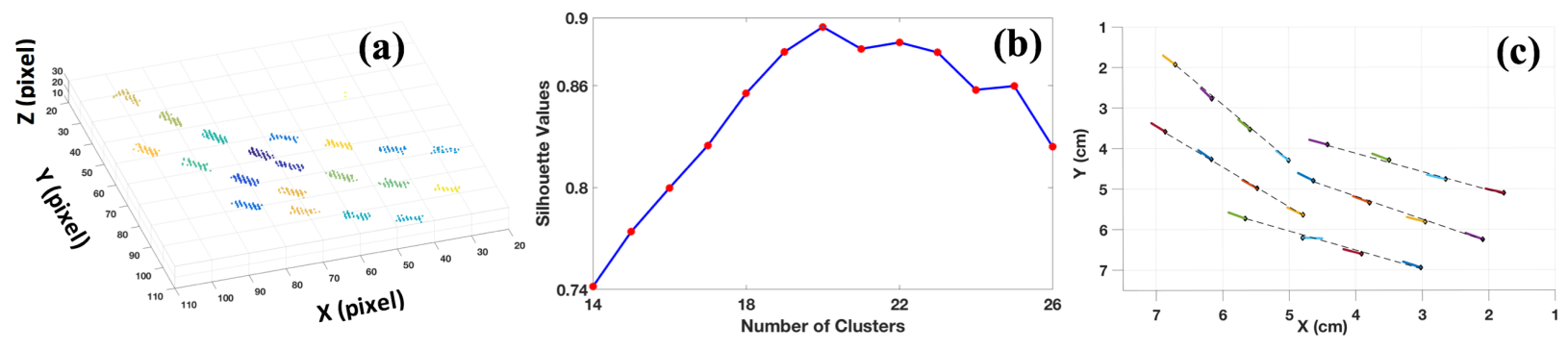

Figure 3.2; (a) the point cloud representation of the thresholded QSM; (b) the calculated k-means based Silhouette values as a function of number of clusters; (c) the estimated medoid (centroids) and orientation of each cluster (seed), the dashed lines represent the actual orientation of each seed strand. 

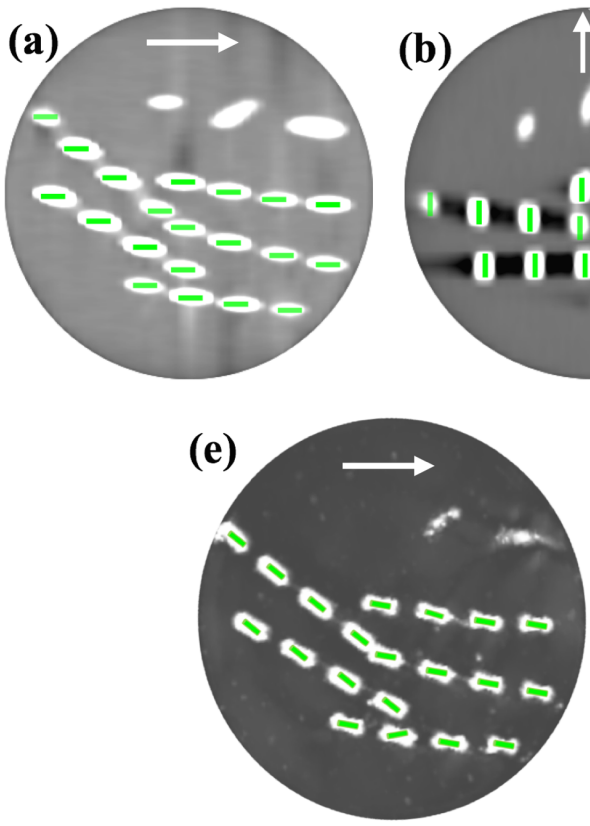

(c)
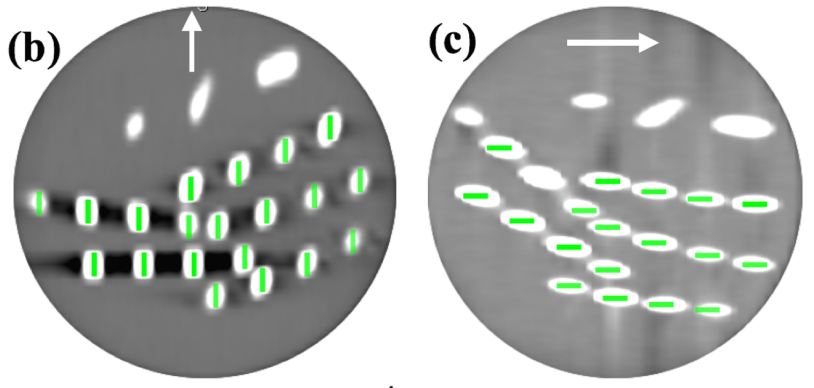

(d)

(f)

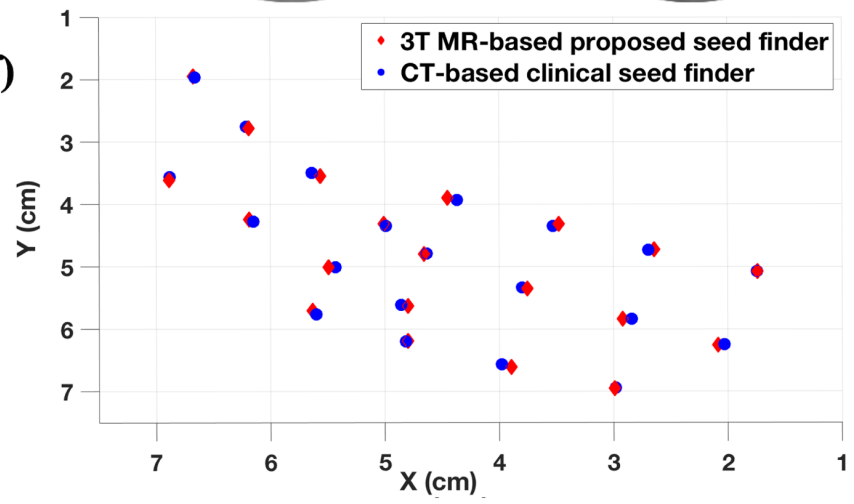

Figure 3.3; The output of the CT-based VariSeed seed finder software for different user-defined number of seeds (a) 20 seeds, parallel scan; (b) 20 seeds, perpendicular scan; (c) 14 seed; (d) 26 seeds. (e) the output of the proposed MR-based seed finder algorithm. (f) the comparison between the centroids calculated using the CT-based clinical software and the MR-based proposed approach. The arrows in CT images indicate the direction of the CT couch during the scan and on MR-based image indicate the direction of the static magnetic field $\left(B_{0}\right)$.

\subsubsection{Performance of the proposed workflow on MR data acquired by 1.5T scanner}

The same post-processing steps (with minor parameter changes) that were used for 3T MR images were applied to images acquired by $1.5 \mathrm{~T}$ scanner to evaluate the performance of the proposed workflow on lower field strength. The calculated susceptibility map using 1.5T MR images is shown in Figure 3.4-b. The estimated centroids using 1.5T and 3T MR scanners along with CT scanner are shown in Figure 3.4-c. The three sets of centroids were compared using oneway ANOVA and no significant difference was found between them $(p<0.01)$. 

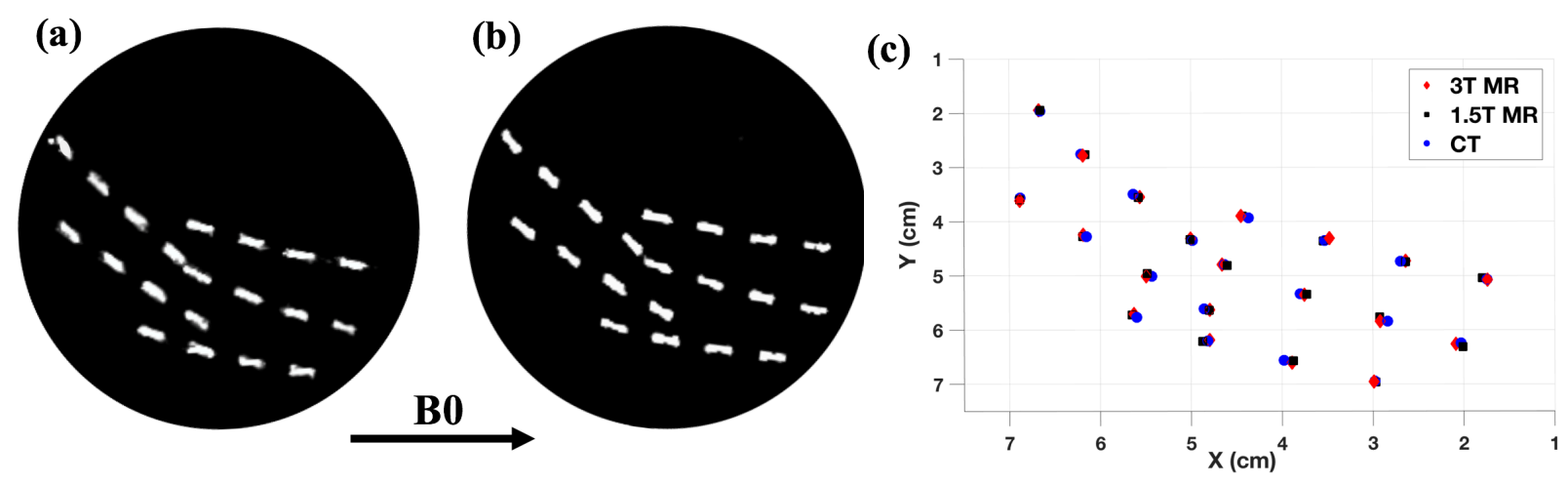

Figure 3.4; The MIP of the calculated QSM using (a) 3T MR images and (b) 1.5T MR images; (c) the comparison between estimated centroids using proposed algorithm based on 1.5T and 3T MR data along with centroids calculated by the clinical CT-based seed finder algorithm.

\subsubsection{Dose Calculation}

Figure 3.5 shows the dose distributions (Gy/U) calculated by the MCNP method in the slice that contained all seeds. Figure 3.5-a shows the dose distribution calculated based on VariSeed centroids however, the orientations of all seeds are assumed to be parallel to the CT couch. In Figure 3.5-b in addition to the centroids calculated by the proposed MR-based pipeline, the estimated orientations are also used in dose calculation.

The absolute difference between the two dose distributions in units of $\mathrm{Gy} / \mathrm{U}$ is shown in Figure 3.4-c. Since there was no significant difference between seed centroids between two methods, any difference stems from seed orientations. The difference in the calculated dose distributions showed that the seed orientations (provided only by the proposed methods) result in a few voxels (ranging from 1 to 6 voxels for each seed corresponding to 1 to $6 \mathrm{~mm}^{3}$ volume) with under- or over-estimated doses (ranging from -8 to $8 \mathrm{~Gy} / \mathrm{U}$ ). 


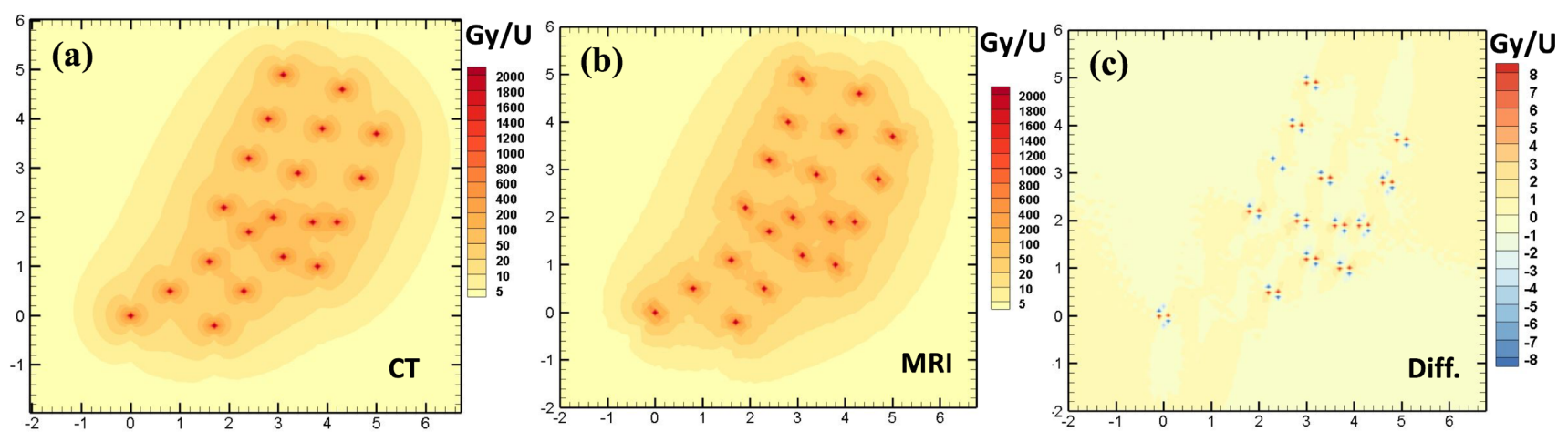

Figure 3.5 (a) dose distribution based on CT-derived positions; (b) dose distribution based on 3T MRderived positions; (c) the absolute difference in dose distributions between $\mathrm{CT}$ and MRI calculated as the absolute difference

\subsection{Discussion ad Conclusion}

Accurate seed depiction and localization during post-implant dosimetry is as critical as precise target delineation. Timely and accurate post implant dosimetry can provide crucial feedback to the clinical team allowing for proper evaluation of the implant quality and possibly enable patient-specific treatment adaptations (whether real-time or not) leading ultimately to optimal treatment delivery. The value of the technology is even greater in focal/salvage treatments where limited target volume is treated since then verifying coverage is even more essential for intended outcome.

The goal of this study was to explore the feasibility of developing an automated MR-only based workflow for accurate localization of seed centers and its orientations. The constraints were to utilize only the clinically widely available MR protocols to generate positive contrast of standard LDR seeds with titanium encapsulation, without the use of uncommon and expensive MR markers, and minimize the scan and post-processing times such to enable easy and widely translatable use. 
In this work, we employed a QSM based approach to generate positive contrast for paramagnetic substances including titanium-encapsulated seeds and calcifications, while having distinctive susceptibility values to easily differentiate them. This quantitative MR technique leads to a contrast level similar to that of CT images in differentiating seeds and calcifications based on CT numbers; the generated susceptibility contrast was employed to differentiate between seeds and calcifications. To our surprise, the clinical CT-based seed finder algorithm did not use CT numbers for seed detection making itself vulnerable to overestimation of number of seeds, e.g., detecting calcifications as seeds if the user-defined number of implanted seeds exceeds the actual number of seeds present. In practice, it has been shown in a study of 1,641 prostate permanent brachytherapy patients that seed migration occurs in $22.7 \%$ of the patients (Nakano et al., 2015); in case of seed loss, the current CT-based algorithm may detect calcifications as seeds; therefore, it is necessary for the physicist to double check all of the seeds and manually correct for under- or over-estimation (if any) of the number of seeds.

It has been shown that accounting for the seeds' orientation in post implant dosimetry has minor impact on the target DVH indices (e.g., D90, V100), however, the dose to the OARs may change by up to $2 \%$ (Collins Fekete et al., 2014). Our dosimetric comparison results suggested that in the CT-based workflow, there were a few voxels in the immediate vicinity of the seeds with severe over/under-estimations resulting from incorrect seed orientations. Although the target/OAR dose calculations were not patient-based in this study, the higher accuracy of dose calculations when considering the seed orientations was evident. Additionally, having accurate orientation information allows for more precise modeling of the inter-seed attenuation effect (Afsharpour et al., 2008; Carrier, Beaulieu, Therriault-Proulx, \& Roy, 2006), thus potentially improving the dosimetric accuracy further. Currently, the CT-based post implant dosimetric calculations do not 
account for seed orientations, but in contrast, MR-based approach presented here successfully showed that seed orientations can be accurately determined (Figure 3.2). If tissue radiological properties such as density and effective atomic number from MR images can be further extractable, this'll provide the most complete information necessary for near-ideal calculation of the true dose distribution (Beaulieu, Tedgren, \& Carrier, 2012; Carrier et al., 2007).

Unlike other proposed MRI-based approaches for prostate post implant dosimetry, the technique proposed here does not require any software or hardware alterations of MRI, uses standard imaging sequences, and hence is easily translatable to any clinic with a different MRI model and field strength between 1.5-3T as it relies on clinical MR protocols without significant acquisition or processing time. In addition, unlike most QSM techniques which require high SNR provided only through high or ultra-high field strength (which may not be available at all centers or practicable on patients), the illustrated efficiency of the proposed workflow on 1.5T and 3T MR images, enhances the advantages of the developed workflow.

Our technique provides higher accuracy for LDR seed localization than CT-based techniques which all lack seed orientations information. In addition, the superior soft tissue contrast of MRI for accurate anatomical delineation makes this workflow potentially the best candidate for postimplant dosimetry although further optimizations may be required for patients.

One of the limitations of this study was that in patients, there might be some signal contamination and loss from other nearby tissues. This has not been taken into account in this work, therefore further patient studies are needed to fine-tune and validate the proposed workflow. Moreover, in this study the number of implanted seeds was very limited and their configuration was rather straightforward compared to a realistic scenario which involves more number of seeds 
(60-100) with more complex configurations (non-spaced or clumped); this work is considered as the methodology development and the subsequent papers will investigate the feasibility of the proposed method in realistic configurations and patients.

The promising results of this study suggest that upon validation of the technique on realistic cases, the proposed MR-based workflow has the potential to replace the conventional CT-based or CT-MRI fusion based methods just by adding a few minutes of scan time to the conventional T2weighted MRI and achieve both superior soft tissue contrast as well as accurate seed localization. Other than post-implant dosimetry of LDR brachytherapy seeds, there are many other potential applications for the developed technique such as depiction and localization of paramagnetic objects such as HDR source/catheter positioning and plan verification for real-time MR-guided brachytherapy and reconstructing titanium based applicators and needles. The feasibility of employing our technique in these other clinical applications will be investigated in the near future.

\subsection{Acknowledgments}

We would like to thank IsoAid Company for providing brachytherapy seeds. This study was supported by the NSERC CGS-D grant. 


\section{CHAPTER 4}

\section{FEASIBILITY OF AN MRI-ONLY WORKFLOW FOR POST- IMPLANT DOSIMETRY OF LOW-DOSE-RATE (LDR) PROSTATE BRACHYTHERAPY IN PRESENCE OF CALCIFICATIONS}

Published in Brachytherapy Journal: Nosrati R, Song WY, Wronski M, Pejović-Milić A, Morton

G, Stanisz G. "Feasibility of an MRI-Only workflow for post-implant dosimetry of low-dose-rate (LDR) prostate brachytherapy: transition from phantoms to patients". Brachytherapy 2019 June

$$
\text { pii } S 1538-4721(19) 30124-2 .
$$

Authors' contributions: R. Nosrati designed and carried out the experiments, designed and performed the post-processing algorithm and drafted the manuscript. M. Wronski helped with CT scans and critically reviewed the manuscript. G. Morton generated brachytherapy plans for phantoms and implanted the prostate phantoms with dummy seeds, referred patients for clinical evaluation, provided advice throughout the course of this work and assessed the manuscript critically. A. Pejovic-Milic assessed the manuscript critically and provided critical feedback. G. Stanisz financed this project and provided advice throughout the project as well as critical feedback to the content, experimental design and the manuscript. 


\subsection{Abstract}

\subsubsection{Purpose}

The lack of positive contrast from brachytherapy seeds in conventional MR images remains a major challenge towards an MRI-only workflow for post-implant dosimetry of Low-Dose-Rate brachytherapy. In this work, the feasibility of our recently proposed MRI-only workflow in clinically-relevant scenarios is investigated and the necessary modifications in image acquisition and processing pipeline are proposed for transition to the clinic.

\subsubsection{Materials and Methods}

Four prostate phantoms with a total of $321 \mathrm{I}-125$ implanted dummy seeds and three patients with a total of 168 implanted seeds were scanned using a gradient echo sequence on $1.5 \mathrm{~T}$ and $3 \mathrm{~T}$ MR scanners. Quantitative Susceptibility Mapping (QSM) was performed for seed visualization. Prior to QSM, the seed-induced distortion correction was performed followed by edge enhancement. Seed localization was performed using spatial clustering algorithms and were compared to CT. Also, feasibility of the proposed method on detection of prostatic calcifications was studied.

\subsubsection{Results}

The proposed susceptibility-based algorithm generated consistent positive contrast for the seeds in phantoms and patients. All the 321 seeds in the four phantoms were correctly identified; the MR-derived seeds centroids agreed well with CT-derived positions (average error $=0.5 \pm 0.3$ $\mathrm{mm}$ ). The proposed algorithm for seed visualization was found to be orientation invariant. In patient cases, all seeds were visualized and correctly localized (average error $=1.2 \pm 0.9 \mathrm{~mm}$ ); no 
significant differences between DVH parameters were found. Prostatic calcifications were depicted with negative contrast on QSM and spatially agreed with CT.

\subsubsection{Conclusions}

The proposed MRI-based approach has great potential to replace the current CT-based practices. Additional patient studies are necessary to further optimize and validate the workflow.

\subsection{Introduction}

Permanent implantation of low-dose-rate (LDR) brachytherapy seeds is a well-established treatment modality for patients with localized prostate cancer. The American Brachytherapy Society (ABS) and the Groupe Europeen de Curietherapie-European Society for Therapeutic Radiology and Oncology (GEC-ESTRO) recommends evaluating the quality of the implant and the dose distribution within 60 days of implantation using standard computed tomography (CT)(Ash et al., 2000; Davis et al., 2012). Seeds have excellent positive contrast on CT images, however, due to the relatively poor soft tissue contrast in $\mathrm{CT}$, anatomical delineation is very challenging and significant inter-observer and intra-observer variabilities have been reported in the CT-based approach(De Brabandere et al., 2013; Dubois et al., 1998; W. R. Lee et al., 2002).

It is widely accepted that magnetic resonance imaging (MRI) is a better imaging modality for delineation of the prostate and the nearby organs at risk (OARs), however, due to the lack of MR signal from the seeds, they appear as dark voids on conventional MR images which are indifferentiable from other such voids associated with calcifications, air cavities, and blood vessels. Thus, CT and MRI are complementary modalities to provide both clear seed identification 
as well as high contrast anatomical visualization of the prostate. Clinical workflows employing CT-MRI registration taking advantage of both imaging modalities is currently implemented at many centers, however this involves additional resources, patient transfers and image registration error(Dehghan et al., 2016; Kunogi et al., 2015; Polo et al., 2004b). An MRI-only workflow for post-implant dosimetry of LDR brachytherapy seeds thus would be an ideal solution for many clinics.

Although several MRI-only approaches have been proposed for seed depiction and localization, none of them have demonstrated robust performance translatable to the clinic. Zijlstra et al. have investigated the feasibility of an MRI-based workflow in which the seed-induced magnetic field distortions were simulated at different orientations and were used for template matching in realistic cases; although the proposed method has acceptable performance in detection of spaced seeds (average error of $0.8 \pm 0.4 \mathrm{~mm}$ ), by average $33 \%$ of the clumped seeds were not identified due to complexity of the field distortion around those seeds(Zijlstra et al., 2017). In addition, the radiation oncologists prefer to clearly observe the seeds for verification of the post-planning seed finder algorithm but the proposed method is not able to portray the seeds with positive contrast. Other studies have proposed new pulse sequences such as IRON and co-RASOR that utilize susceptibility-induced signal pileup around seeds to generate positive contrast, however, these methods require non-clinical pulse sequences and the reconstructed positive contrast seed is either diffused around the seed position or strongly depends on seed orientation(H. de Leeuw et al., 2013; Kuo et al., 2010a; Stuber et al., 2007). Susceptibility-based positive contrast and quantitative susceptibility mapping (QSM) for seeds visualization has also been studied but the proposed methods rely on non-clinical MR pulse sequences or scanners; moreover, the quality of the reconstructed seed images using these methods is significantly worse than that on CT and seed 
visualization is orientation-dependent(Dahnke et al., 2008; Dong et al., 2015; Shi et al., 2017). A novel MR marker (C4) has also been proposed as a spacer between seeds on a strand which is visible on MR images. Although C4 markers appear with positive contrast on MR images, there are several limitations associated with their application which include: limited availability and higher cost compared with standard stranded seeds, necessity of endorectal coil for optimal marker visualization, limited usage for clumped and non-stranded seed applications and seed localization error due to lack of positive contrast from the seeds rather than spacer (Frank et al., 2008; T. Y. Lim, Kudchadker, Wang, Bathala, et al., 2016; T. Y. Lim, Stafford, Kudchadker, Sankaranarayanapillai, Ibbott, Rao, Martirosyan, \& Frank, 2014b; Martin, Ma, et al., 2017).

Recently Nosrati et al. (Nosrati et al., 2018) have proposed an MRI-only pipeline based on quantitative susceptibility mapping (QSM) and unsupervised machine learning (ML) for seed visualization and localization in a simple agar phantom with small number of seeds. In that study data clustering which is the most well-known unsupervised ML algorithm was used for spatial localization of the seeds. Unlike supervised ML, unsupervised learning does not require labeled training data and the algorithm learns to partition the data into several clusters based on their similarities. The method proposed in (Nosrati et al., 2018) showed high efficiency in a simple agar phantom however a number of limitations needed to be addressed including: realistic seed configurations, seed orientation dependence of the algorithm, absence of clumped seeds, correction of the seed-induced MR image distortions, absence of any patient related physiological noise, unrealistic uniformity of the imaging volume, and absence of OARs for dosimetric analysis. 


\subsection{Materials and Methods}

\subsubsection{Phantoms}

Four realistic prostate phantoms (CIRS Inc. 053L model) which contained a simulated prostate gland with three lesions as well as a simulated urethra and rectum were used. Phantoms were imaged using trans-rectal ultrasound (TRUS) and the preplans (needle loading plans) were generated accordingly in the VariSeed ${ }^{\mathrm{TM}}$ software (Varian, Palo Alto, CA). The phantoms were implanted under TRUS image guidance comprising a total of 321 I-125 dummy stranded seeds (IsoAid Advantage ${ }^{\mathrm{TM}}$ ). The needle loading plans included several double and triple loaded (nonspaced) seeds.

\subsubsection{Patients}

Three patients participated in this study; Two patient received standard whole gland brachytherapy with 78 and 66 implanted stranded seeds (I-125, IsoAid Advantage). The third patient was a participant in a phase I/II clinical trial of focal brachytherapy with 24 stranded seeds (I-125, IsoAid Advantage) implanted in the MR defined Dominant Intraprostatic Lesion (DIL). There were a total of eight double loading (16 clumped seeds in total) in the patients' LDR treatment plan. Both whole gland LDR patients had prostatic calcifications confirmed with CT. Currently, at the center that this study was conducted, the standard of care for post-implant dosimetry of prostate LDR brachytherapy requires both CT and MR scan within 1 month after seed implantation procedure. In addition to the standard of care which included the turbo spinecho T1-, T2-weighted and diffusion-weighted MRI, for MRI-based seed identification these patients underwent a gradient echo sequence during the same MR scan. 


\subsubsection{Data Acquisition Parameters}

The phantoms were scanned with a 3D multi echo gradient recalled echo (ME-GRE) sequence at both 1.5T MRI (Philips Ingenia) and 3T MRI (Philips Achieva) scanners using an 8channel head coil array. The pulse sequence parameters on $3 \mathrm{~T}$ scanners were: $\mathrm{TE} 1 / \mathrm{TR}=2.3 / 15.7 \mathrm{~ms} ; \quad$ number $\quad$ of $\quad$ echoes $=3 ; \quad$ flip angle $=15^{\circ} ; \quad \mathrm{FOV}=140 \times 140 \times 100 \mathrm{~mm}^{3}$; resolution $=0.8 \times 0.8 \times 1 \mathrm{~mm} 3$. The pulse sequence parameters on $1.5 \mathrm{~T}$ scanners were: $\mathrm{TE} 1 / \mathrm{TR}=2.2 / 10.3 \mathrm{~ms} ; \quad$ number $\quad$ of $\quad$ echoes $=4 ;$ flip angle $=20^{\circ} ; \quad \mathrm{FOV}=140 \times 140 \times 100 \mathrm{~mm}^{3}$; resolution $=0.8 \times 0.8 \times 1 \mathrm{~mm}^{3}$. The acquisition time was approximately 8 minutes. To evaluate the performance of the proposed method for different orientations of the seeds with respect to the static magnetic field (B0), the phantoms were scanned at three different angles with respect to the B0: $0^{\circ}, 45^{\circ}$, and $90^{\circ}$.

The patients were scanned with a 3T MRI scanner (Philips Achieva) with a 16-channel torso coil (Sense XL Torso). A 3D ME-GRE sequence with fat suppression using "Spectral Presaturation with Inversion Recovery" method (SPIR) and in-phase echo times (for field map estimation and to minimize the chemical shift artifact due to water fat phase cancellation) with the following parameters was utilized: $\mathrm{TE} 1 / \mathrm{TR}=2.3 / 15.7 \mathrm{~ms}$; number of echoes $=3$; flip angle $=15^{\circ}$; FOV $=224 \times 224 \times 120 \mathrm{~mm} 3$; resolution $=1 \times 1 \times 1.5 \mathrm{~mm} 3$. To minimize the motion (breathing) artifact, the anterior-posterior direction was chosen as the frequency encoding direction9. The scan time for the patient was approximately 10 minutes. Due to scan time constraints in patients the slice thickness was increased to $1.5 \mathrm{~mm}$; this should not affect the seed identification accuracy because most of the seed are oriented almost parallel to the axial direction, and the $1.5 \mathrm{~mm}$ axial slice thickness is three times smaller than the length of the seed in that direction $(4.5 \mathrm{~mm})$. 
The CT scanning parameters were set to a standard clinical protocol used for routine postimplant dosimetry evaluations: $120 \mathrm{kVp}, 400 \mathrm{mAs}$, and $3 \mathrm{~mm}$ slice thickness which was reconstructed at $1.5 \mathrm{~mm}$ thickness.

\subsubsection{Geometrical Distortion Correction}

The seed-induced geometrical distortions in MR images were corrected using the magnitude and phase images acquired by the GRE sequence. In general, all MRI pulse sequences generate two kinds of images: magnitude and phase. Magnitude images have the maximum signal to noise ratio (SNR) and are used for most diagnostic purposes in the clinic; phase images are used occasionally in specific applications such as flow measurement and field mapping. To estimate and correct the image distortions, the field map was estimated using phase images then magnitude images were corrected accordingly. The field map estimation and image correction were performed in k-space domain. The k-space data, $y(t)$ and the system matrix, $A(\omega, t)$ were calculated using the MIRT toolbox(Jeffrey A Fessler, n.d.). The method of Matakos et al. for joint estimation of undistorted field map ( $\omega)$ and corrected images (@) was used for distortion correction; the following optimization problem was iteratively solved via the preconditioned conjugate gradients (PCG) algorithm(J.A. Fessler \& Rogers, 1996; Jeffrey A Fessler \& Xed, 1997; A Matakos, Balter, \& Cao, 2014; Antonios Matakos \& Fessler, 2010):

$$
\widehat{\omega}, \hat{\varrho}=\operatorname{argmin}_{\varrho, \omega}\left\|y(t)-A(\omega, t) \varrho^{k}\right\|_{2}^{2}+\lambda_{i}\|C \varrho\|_{2}^{2}+\lambda_{f}\|C \omega\|_{2}^{2}
$$

where $C$ is a second order difference operator and $\lambda_{i}$ and $\lambda_{f}$ are the image and field map 
estimation regularization parameters and were chosen such that the full-width-half-maximum (FWHM) of the point-spread-function (PSF) was 1.1 pixels. An initialization for the field map was introduced as the difference between two consecutive phase images normalized to the $\Delta \mathrm{TE}$.

\subsubsection{Quantitative Susceptibility Mapping (QSM) using MEDI+O}

The diagram in Figure 4.1 shows the proposed workflow for seed visualization and detection. Seed visualization was based on a modified QSM algorithm. In principal, the relative local field disturbance can be approximated as the convolution of the dipole kernel and the local magnetic susceptibility(de Rochefort et al., 2008; Jackson, 1999), therefore theoretically given the local field map, $b(r)$ and the dipole kernel, $d(r)$ the susceptibility, $\chi(r)$ can be calculated in the Fourier domain as following:

$$
\begin{aligned}
& b(r)=d(r) \otimes \chi(r) \stackrel{\text { Fourier Transform }}{\longrightarrow} B(k)=D(k) \times \chi(k) \Rightarrow \chi(k)=B(k) / D(k) \\
& d(r)=\frac{3 \cos ^{2} \theta_{r}-1}{4 \pi r^{3}} \stackrel{\text { Fourier Transform }}{\longrightarrow} D(K)=\frac{1}{3}-\frac{k_{Z}^{2}}{k^{2}}
\end{aligned}
$$

Dipole kernel, $d(r)$ is the magnetic field generated by a unit dipole at the origin which is zero at $\theta=54.57$. Therefore, field to susceptibility inversion problem in equation 2 is ill-posed requires regularization.

The 3D susceptibility map was calculated in the following steps: after distortion correction and edge enhancement, the frequency map $\left(f_{r}\right)$ was estimated using the distortion corrected magnitude $(M)$ and phase $(\theta)$ images obtained at the first echo by solving the following exponential minimization problem: 


$$
f_{r, \theta_{0 r}}=\operatorname{argmin}\left\|M_{r, T E_{1}} e^{i \theta_{r, T E_{1}}}-M_{r, T E_{1}} e^{i\left(f_{r} \times T E_{1}+\theta_{r, 0}\right)}\right\|_{2}^{2}
$$

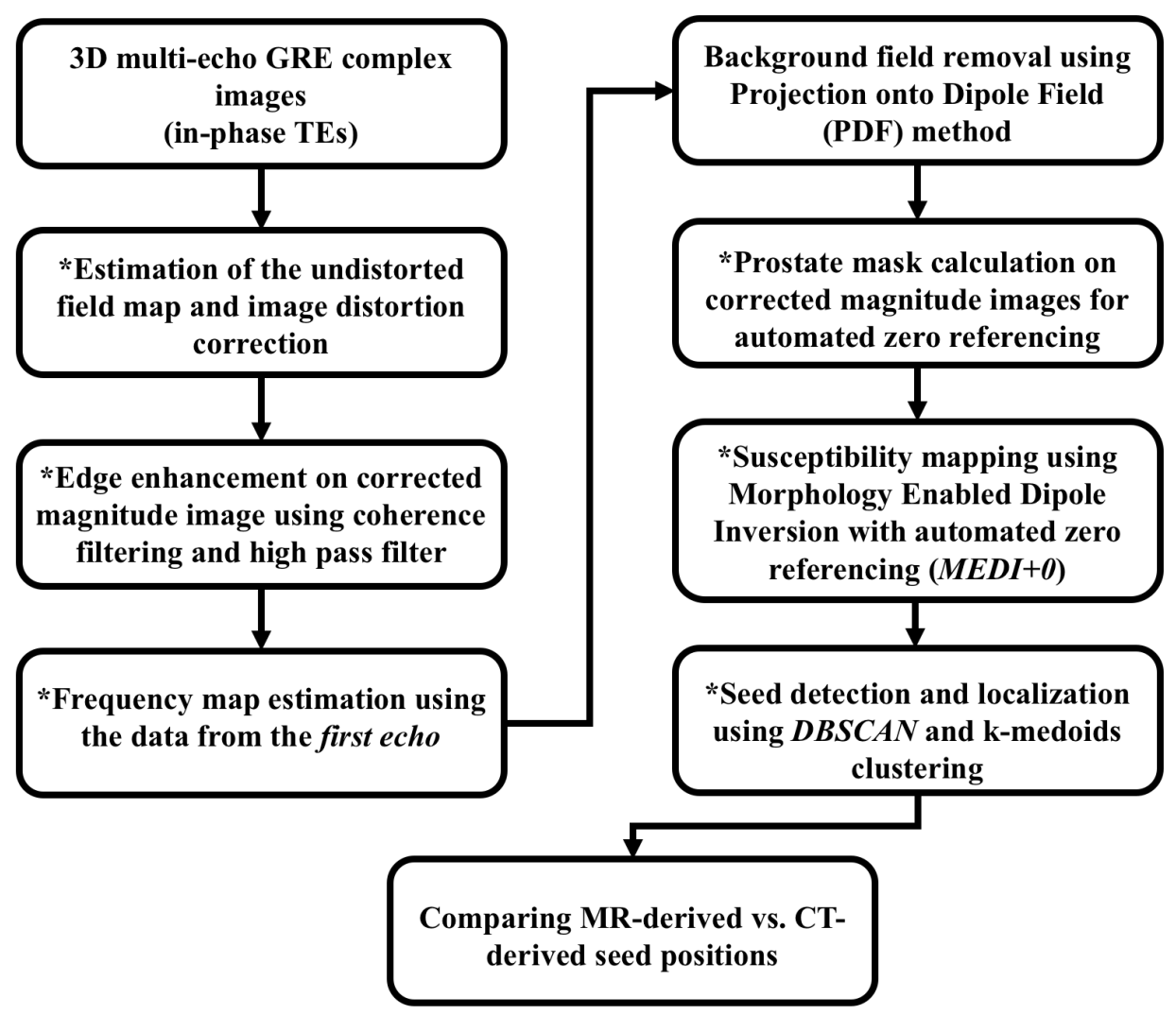

Figure 4.1; The schematics of the proposed workflow for brachytherapy seed visualization and localization. The steps shown by the "**" indicate the proposed post-processing modifications compared to the previously proposed method (Nosrati et al., 2018).

The background field was calculated using projection onto dipole field (PDF) method (T. Liu, Khalidov, et al., 2011) and was subtracted from the frequency map. Finally, the susceptibility map was calculated using morphology enabled dipole inversion with automated prostate zero referencing $(\mathrm{MEDI}+0)$. The following regularization problem was solved iteratively using 
conjugate gradient method (J. Liu et al., 2012b; T. Liu et al., 2013; Z. Liu, Spincemaille, Yao, Zhang, \& Wang, 2018):

$$
\chi_{r}=\operatorname{argmin}_{\chi_{r}}\left\|W\left[e^{i D_{r} \chi_{r}}-e^{i f_{r}}\right]\right\|_{2}^{2}+\lambda_{1}\left\|G_{M} G_{\chi}\right\|_{1}+\lambda_{2}\left\|M\left[\chi_{r}-\overline{\chi_{P}}\right]\right\|_{2}^{2}
$$

where $D_{r}$ is the Fourier domain dipole kernel; $f_{r}$ is the off-resonance frequency map (after background field removal); $W$ is a weighting matrix calculated using a binary mask normalized to the noise standard deviation; $W$ was tuned at each iteration using the Model Error Reduction Through Iterative Tuning (MERIT) technique(T. Liu et al., 2013); $G$ is the gradient operator; $M$ is a binary mask calculated by thresholding the undistorted magnitude images to roughly segment the prostate and muscles for zero referencing; $\overline{\chi_{P}}$ is the average estimated susceptibility within the segmented volume. The L1 regularization term minimizes the number of voxels corresponding to an edge in estimated susceptibility map but not an edge in the magnitude(J. Liu et al., 2012a; T. Liu, Liu, et al., 2011; Yi Wang \& Liu, 2015). The second L2 regularization term enforces susceptibility homogeneity within the prostate and reduces the artifacts around the seeds in the reconstructed QSM(Z. Liu et al., 2018).

To achieve optimal seed reconstruction (in both shape and size) through QSM, prior to dipole inversion the edges of the magnitude images were enhanced using the coherence filtering followed by a Fourier-based high-pass filter (Kroon \& Slump, 2009). 


\subsubsection{Seed Localization and Dosimetric Comparison}

The estimated susceptibility maps were thresholded at $20 \%$ of the maximum susceptibility value for each subject. Seed centroids were identified using unsupervised machine learning algorithms in MatLab based on density-based spatial clustering of applications with noise (DBSCAN) followed by the K-medoid clustering for connected clusters (clumped seeds)(Ester, Kriegel, Sander, \& Xu, 1996; Park \& Jun, 2009; Sander et al., 1998). The disconnected (spaced) seeds were localized using DBSCAN algorithm. The average number of points in each cluster for an individual seed was calculated; then K-medoid clustering was performed on clusters which exceed the average number of points to localized clumped (non-spaced) seeds. Seed orientations were not taken into account for dosimetric analysis.

The seed localization on CT images was performed in MIM software (MIM Symphony Dx ${ }^{\mathrm{TM}}$ ). To compare the MR-based seed positions with that of CT, the estimated seed centroids using the proposed MR-based workflow were registered to those calculated by the clinical CT-based software (MIM Symphony). Prior to registration, the MRI (QSM and T2weighted) and CT images were registered on MIM Symphony and the agreement between the positive contrast seed positions on CT and MR (QSM) were visually (qualitatively) inspected. To register the MR and CT-derived seed positions, first one representative seed centroids was chosen on QSM and the corresponding seeds was identified on CT (for instance the most superior/inferior seed) and a rough (initial) translation matrix (from MRI to CT) was calculated between the two points. The initial estimated translation was applied to all MR-based centroids and then an automated registration was performed for fine tuning the registration. The final rotation and translation matrix between the two point clouds was calculated using a rigid registration framework based on Iterative Closest Point (ICP) algorithm(Besl \& McKay, 1992). 
To analyze the dosimetric effect of the estimated MR-based seed positions versus the conventional CT-based approach, prostate, urethra (only in phantoms), and rectum were contoured on CT images and accumulative dose volume histograms (DVH curves) were calculated in MIM software first with CT-derived seed positions and subsequently using the MR-derived positions. The dose to $90 \%$ of the prostate volume (D90), the prostate volume that receives 100, 150 and $200 \%$ of the prescribed dose $\left(\mathrm{V}_{100}, \mathrm{~V}_{150}\right.$ and $\left.\mathrm{V}_{200}\right)$, and the dose to $2 \mathrm{~cm}^{3}$ of the rectum and urethra $\left(\mathrm{D}_{2 \mathrm{cc}, \text { rectum, }} \mathrm{D}_{2 \mathrm{cc} \text {,urethra }}\right)$ were compared between the two methods.

Two-tailed paired sample t-test was used to evaluate the significance of the difference between CT- and MRI-derived seed positions and dosimetric parameters. Statistical analysis was performed using the SPSS software (IBM SPSS statistics 24). Statistical significance was assumed to be at $\mathrm{p}<0.05$. To assess the agreement between the seed positions using the proposed algorithm vs. the standard CT-based method the Bland-Altman analysis with $95 \%$ confidence interval (CI) was performed.

\subsection{Results}

\subsubsection{Phantom results}

\subsubsection{Seed Visualization at Different Orientations with Respect to BO}

One of the prostate phantoms with 70 implanted seeds and the associated raw magnitude and phase MR images are illustrated in Figure 4.2a-d. The maximum intensity projections (MIP) reconstruction of the calculated susceptibility maps at three different angular positions as well as CT images are shown in Figure 4.2e-h. As shown in Figure 4.2e-g, the proposed seed visualization algorithm was not affected by the seed orientation and changing the orientation of the phantoms 
(seeds) did not affect the seed reconstruction. In phantoms, the new proposed algorithm and the method proposed in(Nosrati et al., 2018) generated visually similar susceptibility maps. However, the distortion correction step reduced the seed localization error which is discussed in the following section.

\subsubsection{MR-based Seed Localization and Dosimetric Analysis}

All 321 implanted seeds in phantoms were correctly detected. Figure 4.3a-d shows comparisons between seed positions estimated by the proposed MR-based algorithm and the clinical CT-based seed finder in all four phantoms. The maximum and average distance $+/-$ standard deviation between the CT and MR-derived positions for all 321 seeds were $1.3 \mathrm{~mm}$ and $0.5 \pm 0.3 \mathrm{~mm}$ respectively whereas, without distortion correction and applying the K-means clustering as proposed in(Nosrati et al., 2018) one of the seeds in the triple loaded strand was mis-detected and the maximum and average errors (excluding the mis-detected seed) were $2.9 \mathrm{~mm}$ and $0.9 \pm 1.1$ respectively.

The differences in seed positions for each phantom approximated to a Gaussian distribution that was confirmed by Kolmogorov-Smirnov normality test $(\mathrm{p}>0.3)$. Bland-Altman analysis of MRbased compared to CT-derived seed centroids revealed a very small bias of $-0.0005 \mathrm{~mm}$ with narrow [-0.43 +0.43] mm 95\% limits of agreement; As shown in Figure 4.3e, only two out of 321 seeds were marginally out of the $95 \%$ confidence interval level.

The DVH parameters including prostate $\mathrm{D}_{90}, \mathrm{~V}_{100}, \mathrm{~V}_{150}$ and $\mathrm{V}_{200}$ as well as $\mathrm{D}_{2 \text { cc,rectum }}$ and

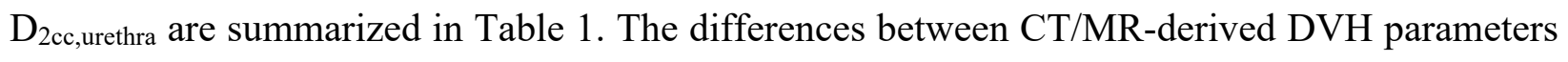
were less than $1.5 \%$. The correlation between MR and CT-based DVH indices is illustrated in Figure $4.3 \mathrm{f}$ showing excellent agreement between them $\left(\mathrm{R}^{2}>0.99\right)$. 


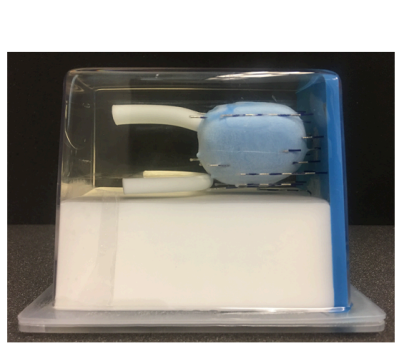

(a)

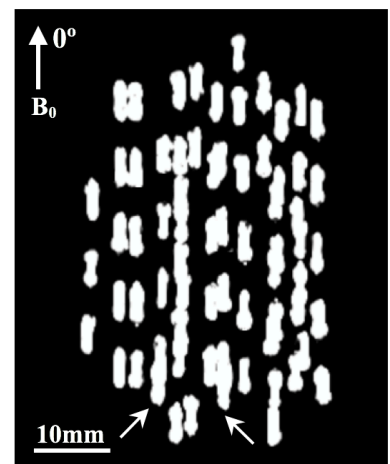

(e)

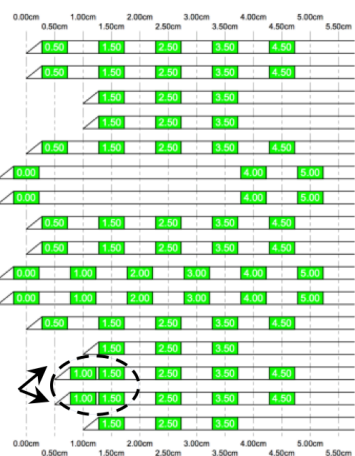

(b)

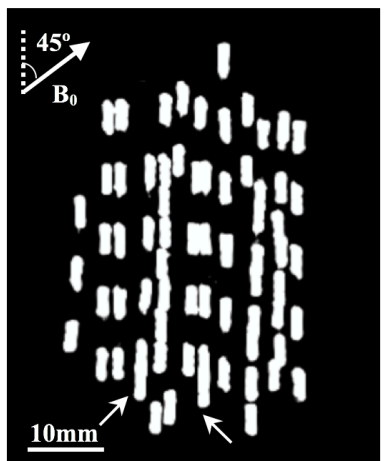

(f)

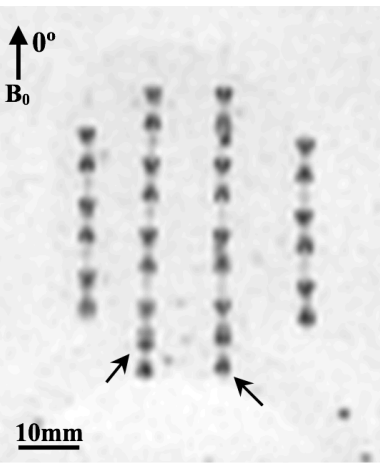

(c)

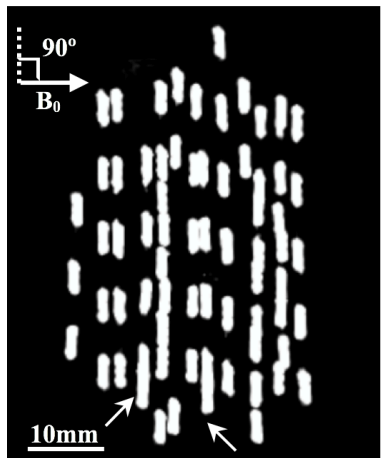

(g)

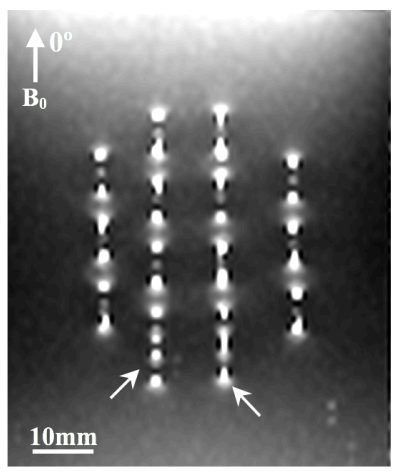

(d)

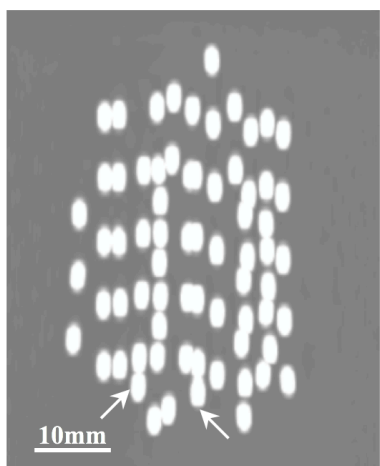

(h)

Figure 4.2; (a) one sample phantom with 70 implanted seeds; (b) the seed implantation plan (needle loading) which contained four non-spaced seeds shown by the arrows; (c) the Minimum Intensity Projection (MinIP) of the magnitude image from 20 slices at the shortest TE; (d) the Maximum Intensity Projection (MIP) of the phase image from 20 slices at the shortest TE. The full thresholded MIP of the calculated susceptibility maps with the phantom orientated at (e) 0 degree, (f) 45 degrees and (g) 90 degrees with respect to the static magnetic field $\left(B_{0}\right)$; (h) the MIP of the CT image of the same phantom. The arrows in b-g indicate the non-spaced (double-loaded) seeds. 
(a)

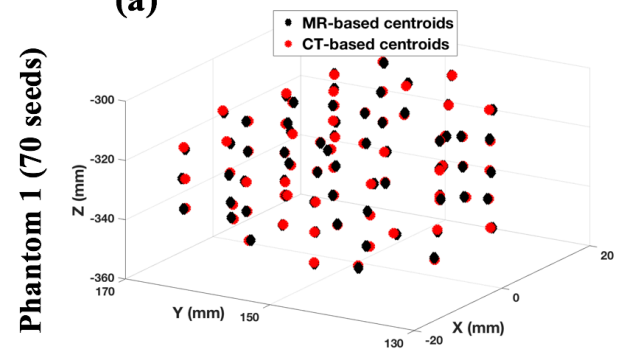

(c)

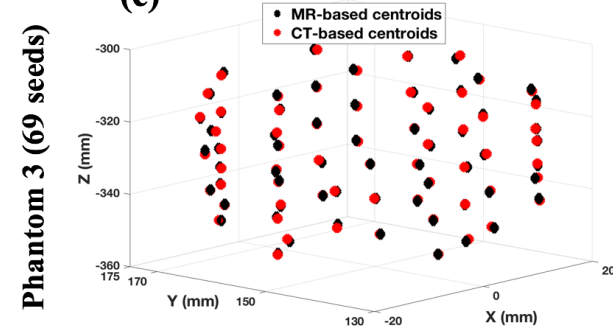

(e)

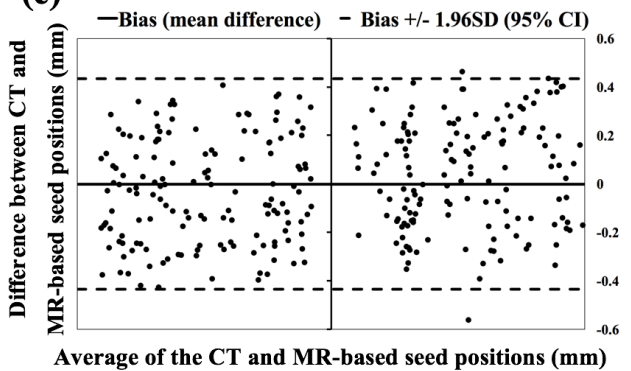

(b)

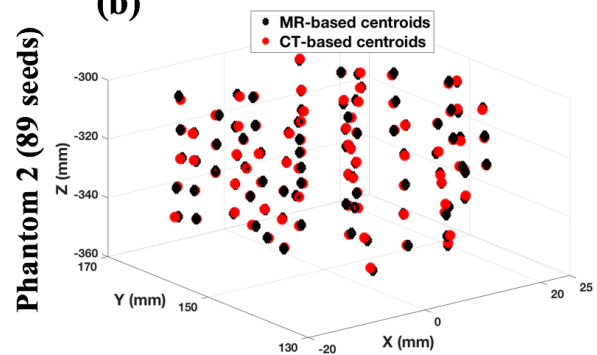

(d)
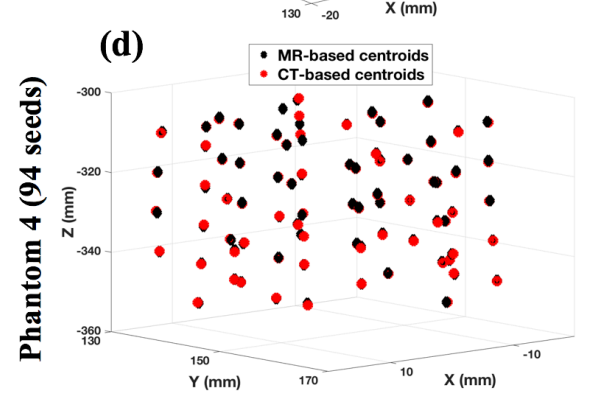

(f)

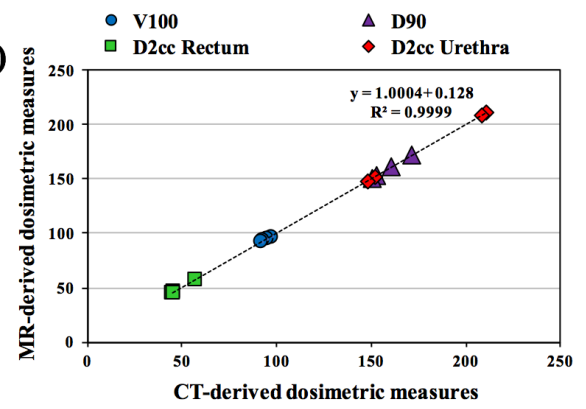

Figure 4.3; (a-d) Comparison between clinical CT-based (MIM Symphony) and the proposed MR-based seed localizer algorithms on each of four phantoms; (e) The Bland-Altman plot for CT and MR-derived seed positions for all 321 seeds; (f) The correlation between MR and CT-derived DVH parameters.

\subsubsection{Patient results}

\subsubsection{MR-based Seed Visualization}

Figure 4.4a-d illustrates different steps of the proposed pre-QSM processing pipeline for the

patient with 78 implanted seeds. The estimated distortion map which was calculated as the field map normalized to the bandwidth scaled by the voxel size is shown in Figure 4.4a; the maximum 
distortion was $1.1 \mathrm{~mm}$ observed near the seeds. The difference between distorted and undistorted magnitude images is shown in Figure 4.4c.
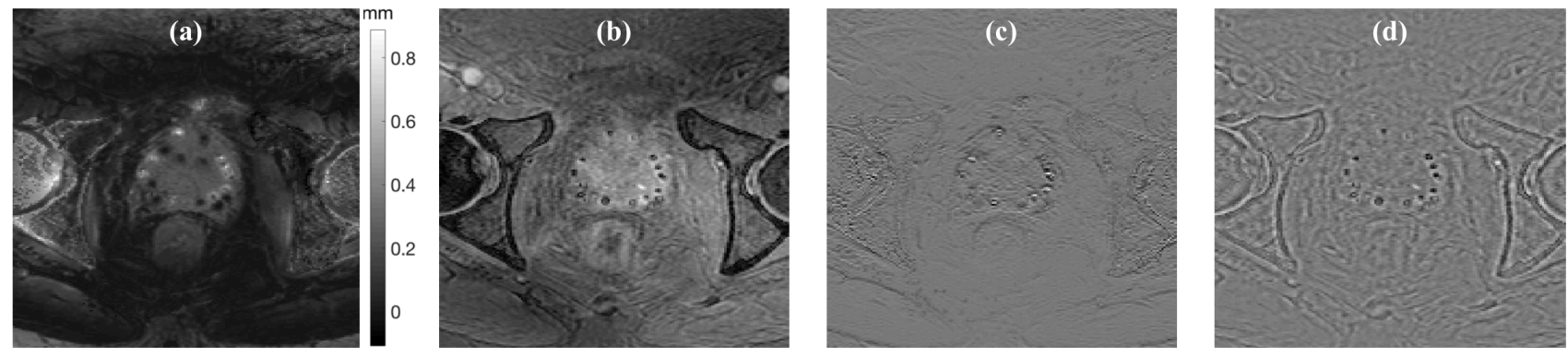

Figure 4.4; (a) The estimated distortion map; (b) distortion corrected magnitude image; (c) difference between corrected and uncorrected magnitude images; (d) corrected magnitude image after edge enhancement.

Figure 4.5 show the CT image, corrected magnitude and phase images, prostate and muscle mask for zero referencing, and the MEDI, MEDI +0 with and without distortion correction results for one axial cross section of the prostate.

The effectiveness of each of the proposed modifications in the QSM pipeline are demonstrated in Figure 4.4i-1. The efficiency of algorithm proposed in(Nosrati et al., 2018) was significantly degraded in patients compared to the phantoms and seed reconstruction was unsuccessful particularly near the prostate boundaries (Figure 4.4i). The key changes in acquisition and post-processing pipeline were: changes to the MR pulse sequence parameters, seed-induced distortion correction, eliminating the phase unwrapping step, using only the first echo for frequency map estimation, edge enhancement and optimizing the edge threshold for extracting morphological information from magnitude image (required for MEDI), optimizing regularization parameters in patients, and finally applying prostate zero referencing (MEDI +0$)$. The image postprocessing pipeline took approximately 6 minutes per patient to visualize and localize the seeds. 
The MEDI +0 technique significantly improved the homogeneity of the prostate and muscles while unaffecting the seeds. In addition, the artifacts around the reconstructed seeds in QSM were significantly reduced by applying the MEDI+0 algorithm. Distortion correction improved the spatial accuracy of the susceptibility mapping; the arrow in Figure 4.4k-1 indicates one seed which was slightly shifted in the axial direction after distortion correction.
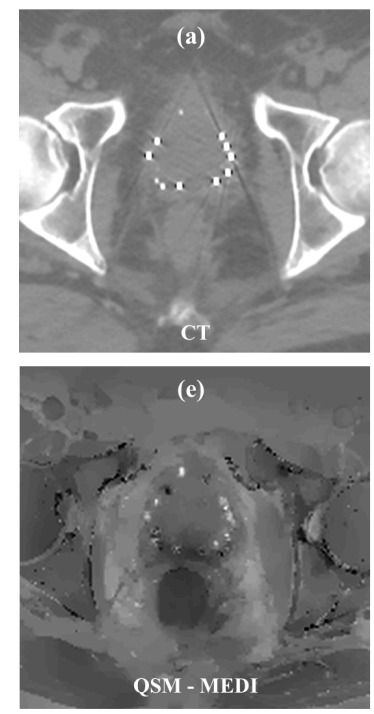
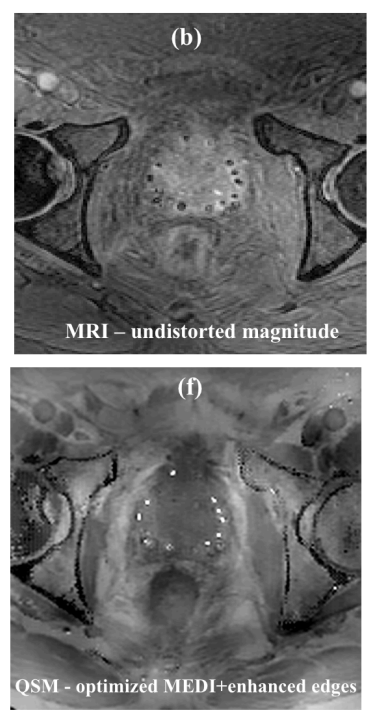
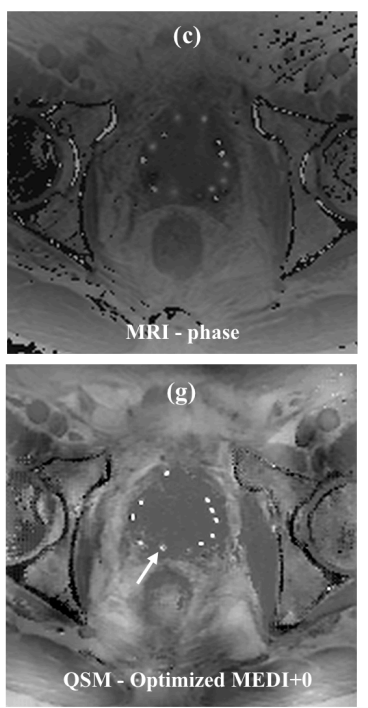
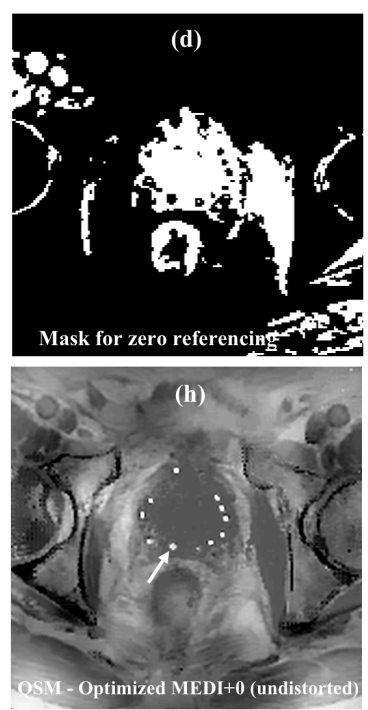

Figure 4.5; (a) For one axial slice of the prostate: (a) CT image; (b) MR magnitude image acquired at $\mathrm{TE}=2.3 \mathrm{~ms}$; (c) $\mathrm{MR}$ phase image acquired at $\mathrm{TE}=2.3 \mathrm{~ms}$; (d) estimated prostate and muscle mask for zero referencing during dipole inversion process; (e) the estimated susceptibility map by applying the exact method proposed in(Nosrati et al., 2018); (f) the estimated susceptibility map using the proposed workflow with edge enhancement but without distortion correction and prostate zero referencing; (g) the estimated susceptibility map using the proposed workflow with MEDI+0 but without distortion correction; (h) the estimated susceptibility map using the proposed workflow with edge enhancement, MEDI+0 and distortion correction.

Figure 4.6 shows the T2 and T1-weighted MR images, MIP reconstruction of the CT, and the processed MR images (QSM) for all three patients. The quality of the seed visualization in processed MR images with the proposed method is comparable to that on CT. It should be noted 
that any observed visual differences between CT and QSM may be due to prostate deformation between two scans as well as the differences in image acquisition parameters (slice thickness) between CT and MRI.

As shown in Figure 4-7 patients with 78 and 66 seeds had prostatic calcifications. On CT

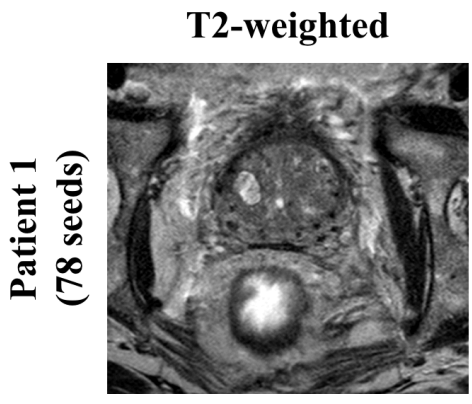

T1-weighted

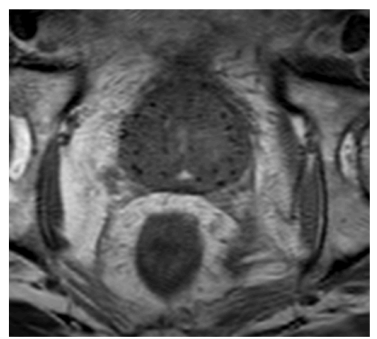

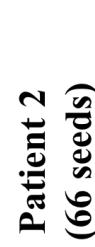
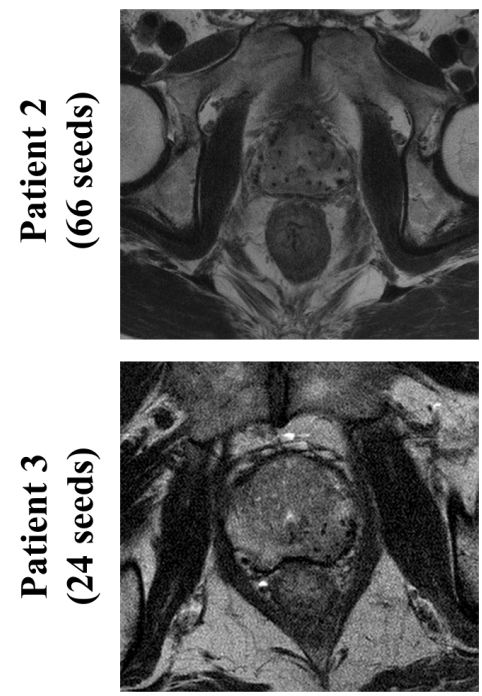

QSM
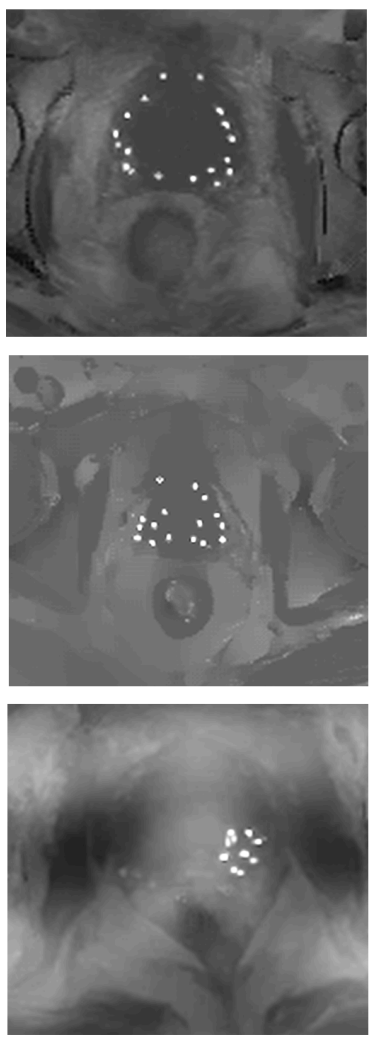

CT
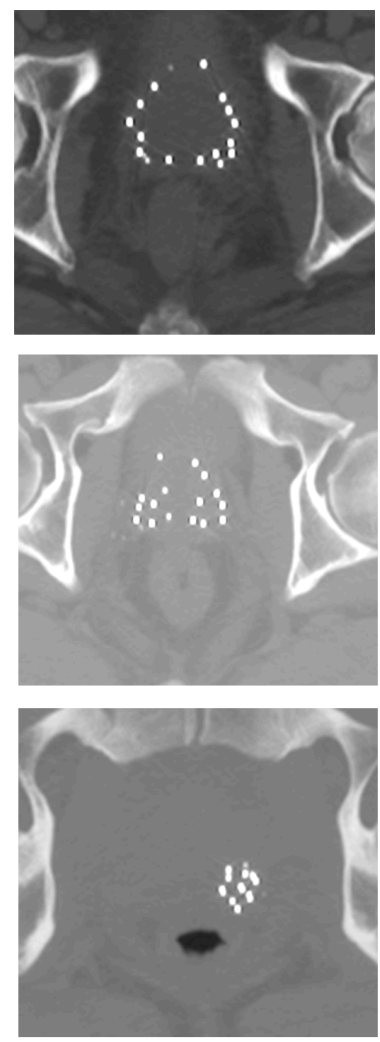

Figure 4.6; T2-weighted MR, T1-weighted MR and the MIP reconstruction of a stack of five slices of CT and QSM of three patients.

calcifications appear with positive contrast like seeds however, given the diamagnetic properties (average magnetic susceptibility of -14 ppm) of calcium carbonate, calcifications are depicted with negative contrast on QSM which makes them easily differentiable from positive contrast paramagnetic seeds. 

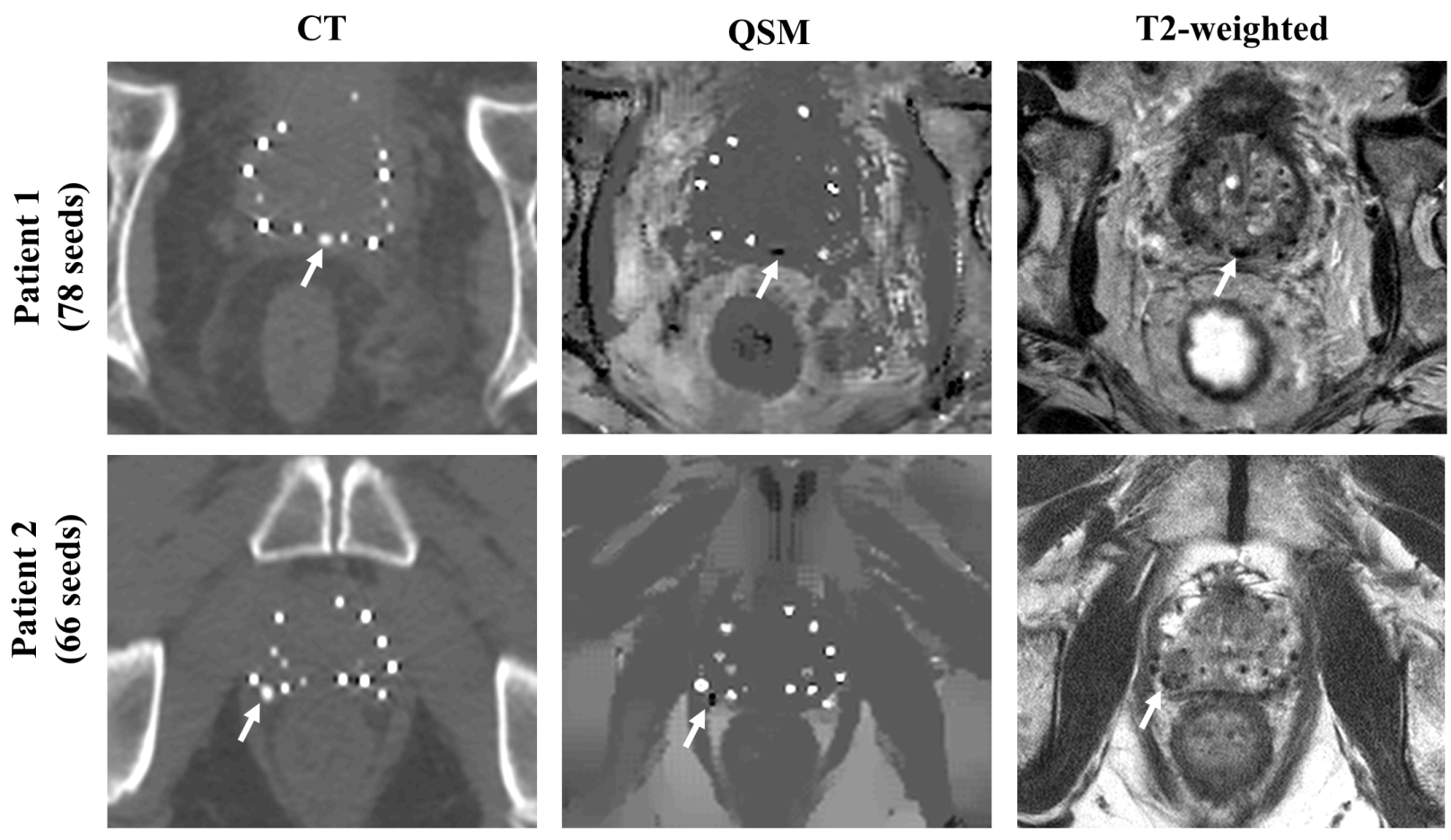

Figure 4.7; CT, QSM and T2-weughted MR images of an axial slice of the prostate which contained a prostatic calcification. The arrows indicate the location of the calcification.

\subsubsection{2. $\quad$ MR-based Seed Localization and Dosimetric Analysis}

The seed The seed localization algorithm identified all 168 seeds with good spatial accuracy and there was no significant difference between MR and CT-based seed positions ( $\mathrm{p}>0.8)$. Figure 4.8a compares the detected seed centroids using the proposed MR-only and the standard CT-based approaches in the patient with 66 seeds; the MRI and CT-derived DVH curves for the same patient are shown in Figure 8b. The comparison between the dosimetric indices in patients is presented in Table 1. The maximum and average distance between MR and CT-derived seed positions were 2.6 $\mathrm{mm}$ and $1.2 \pm 0.9 \mathrm{~mm}$ respectively by applying the proposed algorithm.

The differences in MR and CT-derived seed positions in each patient approximated to a Gaussian distribution which was confirmed by Kolmogorov-Smirnov normality test $(\mathrm{p}>0.1)$. 
Bland-Altman analysis of MR-based compared to CT-derived seed centroids revealed a small bias of $-0.41 \mathrm{~mm}$ with narrow [-1.85 +1.04] $\mathrm{mm} 95 \%$ limits of agreement. As shown in Figure 8c, only four out of 168 seeds were out of the $95 \%$ confidence interval level.
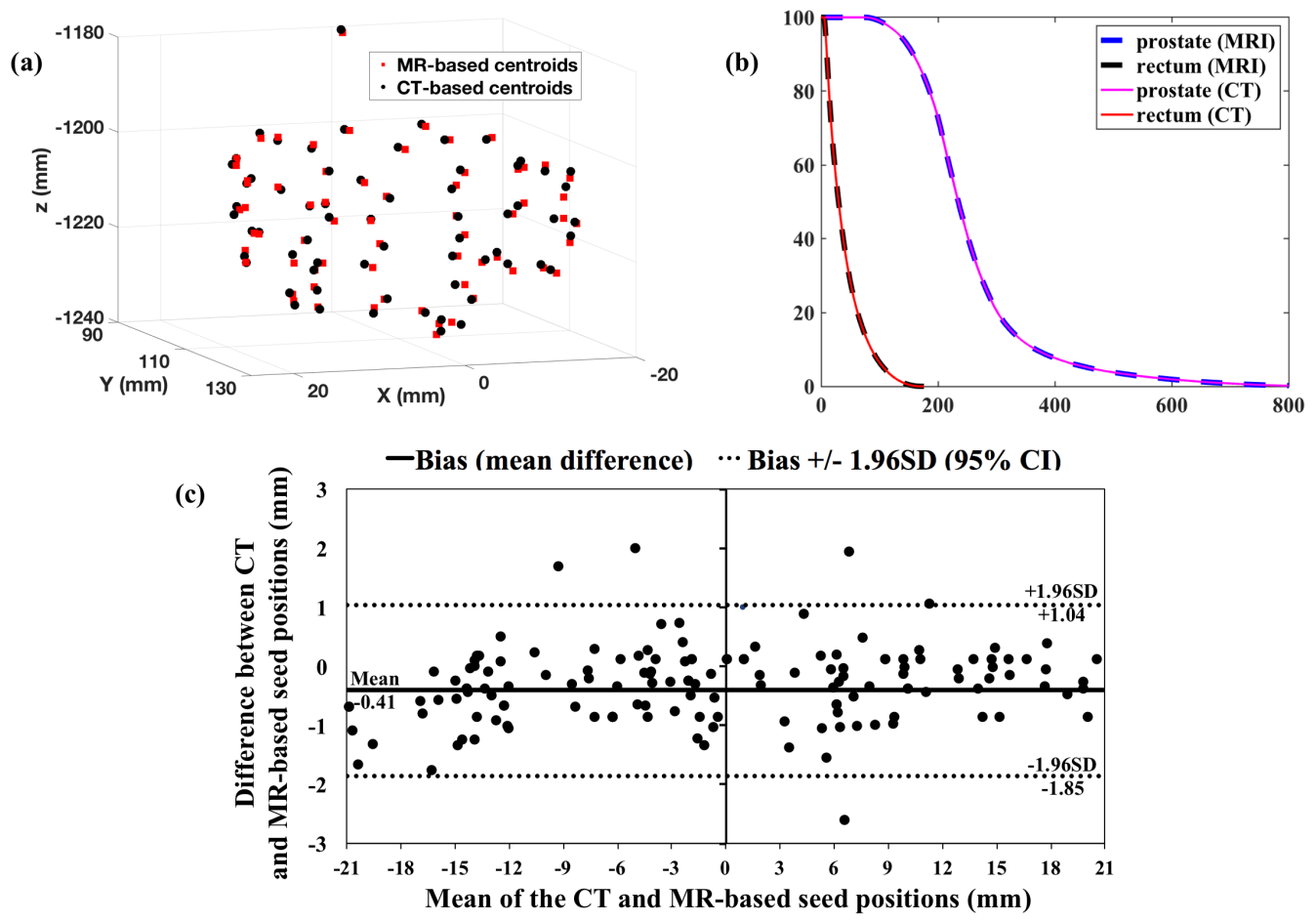

Figure 4.8; Comparison between the proposed MR and CT-derived positions in (a) patient with 78 implanted seeds and (b) patient with 66 implanted seeds; (c) Blant-Altman plots of MR-derived vs. CTderived seed centroids; results; (d) the estimated DVH curves based on CT/MR-derived seed positions for the patient with 78 seeds. 
Table 4.1; The summary of dosimetric parameters obtained from DVH curves calculated using CT and MR-derived seed positions.

\begin{tabular}{|c|c|c|c|c|c|c|c|c|c|c|c|c|}
\hline & \multicolumn{2}{|c|}{ D90 $_{9 y}$ (Gy) } & \multicolumn{2}{|c|}{$V_{100}(\%)$} & \multicolumn{2}{|c|}{$V_{150}(\%)$} & \multicolumn{2}{|c|}{$V_{200}(\%)$} & \multicolumn{2}{|c|}{$D_{2 c c, \text { rectum }}(G y)$} & \multicolumn{2}{|c|}{$D_{2 c c}$, urethra $(G y)$} \\
\hline & CT & MRI & CT & MRI & CT & MRI & CT & MRI & CT & MRI & CT & MRI \\
\hline Phantom 1 & 172.1 & 171.6 & 97.3 & 97.2 & 70.5 & 70.3 & 26.0 & 26.2 & 46.2 & 46.3 & 211.5 & 211.7 \\
\hline$\%$ Difference & \multicolumn{2}{|c|}{$0.3 \%$} & \multicolumn{2}{|c|}{$0.1 \%$} & \multicolumn{2}{|c|}{$0.3 \%$} & \multicolumn{2}{|c|}{$0.7 \%$} & \multicolumn{2}{|c|}{$0.2 \%$} & \multicolumn{2}{|c|}{$0.1 \%$} \\
\hline Phantom 2 & 161.1 & 160.9 & 94.9 & 94.9 & 61.0 & 60.4 & 20.1 & 20.1 & 45.1 & 45.1 & 208.6 & 208.6 \\
\hline \% Difference & \multicolumn{2}{|c|}{$0.1 \%$} & \multicolumn{2}{|c|}{$0 \%$} & \multicolumn{2}{|c|}{$1.0 \%$} & \multicolumn{2}{|c|}{$0.1 \%$} & \multicolumn{2}{|c|}{$0 \%$} & \multicolumn{2}{|c|}{$0 \%$} \\
\hline Phantom 3 & 153.6 & 153.9 & 93.3 & 93.4 & 44.6 & 44.3 & 17.5 & 17.5 & 45.5 & 45.7 & 152.3 & 151.7 \\
\hline$\%$ Difference & \multicolumn{2}{|c|}{$0.2 \%$} & \multicolumn{2}{|c|}{$0.1 \%$} & \multicolumn{2}{|c|}{$0.7 \%$} & \multicolumn{2}{|c|}{$0.5 \%$} & \multicolumn{2}{|c|}{$0.4 \%$} & \multicolumn{2}{|c|}{$0.4 \%$} \\
\hline Phantom 4 & 151.3 & 150.7 & 92.4 & 92.3 & 43.4 & 42.9 & 15.0 & 15.2 & 57.6 & 57.9 & 148.3 & 147.8 \\
\hline$\%$ Difference & \multicolumn{2}{|c|}{$0.4 \%$} & \multicolumn{2}{|c|}{$0.1 \%$} & \multicolumn{2}{|c|}{$1.0 \%$} & \multicolumn{2}{|c|}{$1.4 \%$} & \multicolumn{2}{|c|}{$0.5 \%$} & \multicolumn{2}{|c|}{$0.3 \%$} \\
\hline Patient 1 & 143.3 & 144.5 & 89.2 & 89.6 & 35.6 & 34.2 & 14.2 & 14.3 & 69.8 & 69.0 & - & - \\
\hline$\%$ Difference & \multicolumn{2}{|c|}{$0.8 \%$} & \multicolumn{2}{|c|}{$0.5 \%$} & \multicolumn{2}{|c|}{$1.9 \%$} & \multicolumn{2}{|c|}{$0.8 \%$} & \multicolumn{2}{|c|}{$1.1 \%$} & \\
\hline Patient 2 & 158.3 & 160 & 93.7 & 93.2 & 57.2 & 56.9 & 23.3 & 23.3 & 92.2 & 92.2 & - & - \\
\hline$\%$ Difference & & & & & & $\%$ & & $\%$ & & & & \\
\hline
\end{tabular}

\subsection{Discussions and Conclusions}

In comparison to $\mathrm{CT}$, MR imaging provides improved definition of prostate base and apex, and determination of the prostate rectal interface, where, for example, Mashouf et al.(Mashouf et al., 2016) have found that at a modest contouring uncertainty of $2 \mathrm{~mm}$ expanded from the original prostate contours decreased the D90 by $14.9 \%$. An ideal and powerful approach would be to identify the seeds directly using the MR images thus enabling a true MR-only post-implant plan quality assessment workflow.

Recently Nosrati et al.(Nosrati et al., 2018) have proposed an MRI-only pipeline for brachytherapy seed visualization and localization which was only validated on simple agar phantoms with small numbers of seeds. The performance of that algorithm was significantly degraded in patients therefore the transition of the technique to the clinic required extensive modifications of the MRI acquisition parameters and post-processing algorithm. In summary, in the present study an optimal MRI pulse sequence was proposed, the seed-induced geometrical distortions and intensity artifacts were estimated and corrected; edge enhancement was performed 
on undistorted magnitude images to improve the morphology-enabled dipole inversion process(Adjeiwaah et al., 2018; Khoo et al., 1997; Y. K. Lee et al., 2003; H. Wang, Balter, \& Cao, 2013), susceptibility mapping workflow was optimized and Morphology Enabled Dipole Inversion with prostate tissue zero referencing (MEDI+0) technique was employed, and seed finder algorithm was modified to minimize the localization error in patients. The optimized MRI-only clinical workflow map for post-implant dosimetry of prostate LDR brachytherapy is illustrated in Figure 4.9. In this work, CT was only used to validate the MR-based results however, the proposed workflow is MRI-only.

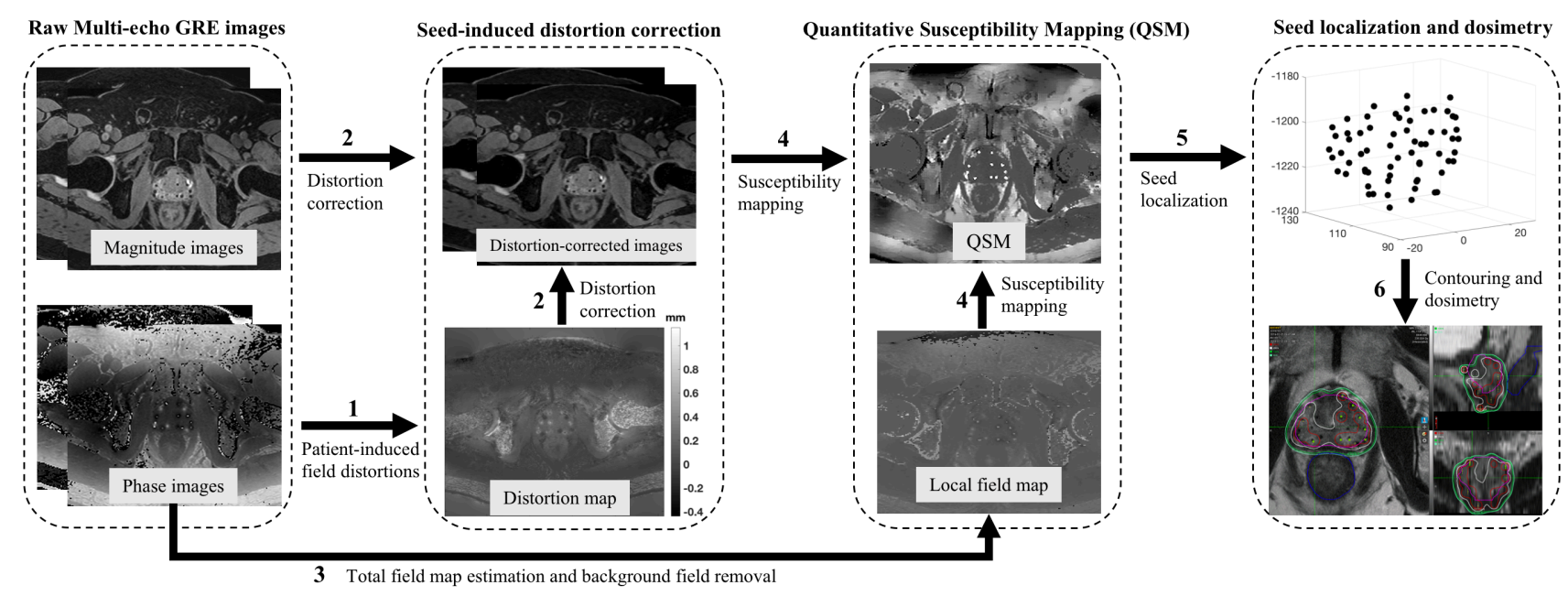

Figure 4.9; Schematics of the proposed MRI-only workflow for clinical implementation

In general, the susceptibility-induced field distortion depends on the direction of the susceptibility source (which in case of brachytherapy seeds is a long cylinder) and the maximum field distortion occurs when the seeds are transverse to the static field while minimum disturbance is when seeds are parallel to the main field(Schenck, 1996); thus theoretically seed visualization and shape reconstruction through QSM is affected by seed orientation. Although susceptibility 
induced phase shift depends on orientation, magnitude images are minimally affected by seed orientation and in this study, we showed that using morphological information from magnitude images (MEDI) helps to reconstruct the seed shape regardless of their orientation which is critical for accurate seed detection.

The patient-induced field inhomogeneities and the resulting image distortions are of main concern in any MRI-based radiation therapy. In radiation treatment planning, few millimeters of distortion may result in significant error in target delineation and dose calculations; hence, geometrical distortions need to be corrected prior to MRI-based treatment planning. Recently, it has been shown that, patient-induced distortions could be significantly larger than those from the system (gradient non-linearity and inhomogeneous B0) in prostate cancer patients(Adjeiwaah et al., 2018). In this study, the pixel bandwidth was relatively high which potentially minimized the image distortion however, due to the presence of high susceptibility implanted seeds, an average distortion of $1.1 \mathrm{~mm}$ was observed around the seeds. The image distortion correction prior to QSM improved the spatial accuracy of seed visualization and localization. Distortion correction could also affect prostate contouring due to susceptibility mismatch between prostate and gas-filled rectum, presence of the seeds at the prostate border, thus joint evaluation of distortion correction on both seed localization and contouring on a large cohort of prostate brachytherapy patients is a potential extension of the present study.

Our results suggest that MEDI +0 significantly improves the homogeneity of the background prostate tissue and reduces artifacts and hypointense shadows at the seed boundaries which are mainly due to the large susceptibility mismatch between seeds and the prostate. The L2regularization of the prostate susceptibility is similar to the L1-regularization term of the gradients and is considered as an additional prior structural constraint that reduces artifacts around the seeds. 
Although DBSCAN algorithm for seed localization handles noise to some extent, enforcing the background (prostate) uniformity improved seed localization accuracy.

This study also showed that the unlike some previous works investigating the feasibility of contrast-enhanced T1-weighted images for MRI-based seed identification(Buch et al., 2014; Ohashi et al., 2012b), the performance of the proposed workflow is similar for both intra-prostatic and extra-prostatic seeds.

The Bland-Altman analysis showed excellent agreement between CT and MRI seed positions in both phantoms and patients. The minor observed differences in seed positions between CT and MRI in patients, could be due to the differences in patient positioning and internal prostate motion between the two scans and may not be the error of the proposed workflow. The small differences in seed positions between CT and MRI did not result in any difference in dosimetric parameters which is in agreement with a previous work showing the low sensitivity of prostate dosimetric parameters to seed localization accuracy(Su, Davis, Furutani, Herman, \& Robb, 2007).

In addition to positive contrast seed visualization using QSM, we showed that the proposed method also visualizes prostatic calcifications with negative contrast without any interference. The difference in MR and CT based dose to prostate was less than $2 \%$. It should be noted that the minor observed differences in dosimetric parameters might have been due to the differences in rectal and prostate positions between two scans.

One limitation in application of this technique in the clinic is the extended MR scan time by about 10 minutes which makes the sequence susceptible to motion artifact. Although the increased scan time may be justified by improved target delineation and overall accuracy, the scan time could be further reduced by optimizing sequence parameters (specially number of signal averages) however, considering the size of the seeds the resolution must be kept as high as possible. Another 
limitation of the present work is the small sample size of patients which needs to be addressed by extending the study to a sufficiently larger cohort of patients (e.g., $\geq 20$ ). The exercise of optimizing the imaging and post processing sequences of a wide-ranging heterogeneous makeup of tissues in a large cohort will certainly improve the robustness of our approach, where the heterogeneous makeup of tissues may present cases of unique signal distortions/losses in the implanted volume. Finally, to assess the accuracy of the MR-based seed localization, rigid registration was performed between CT and MR-based seed centroids. Although applying a rigid registration between seed centroids derived from the two modalities avoided the potential CT-MR image fusion error or bias, it may have masked some systematic errors. The systematic errors of the proposed MRI-based seed visualization and identification algorithm, may be investigated through quantitative analysis of CT-QSM image fusion by positive contrast seed alignment.

The dosimetric comparisons between CT-only, CT+MRI and MR-only approaches in future work will provide a further, valuable insight into the overall importance of the MR-only workflow.

In conclusion, this work provided strong evidence that the proposed MRI-only workflow is feasible and possess a high potential for translation to any clinic.

\subsection{Acknowledgement}

We would like to thank IsoAid Company for providing brachytherapy seeds for phantom studies. R.N. is funded by the NSERC CGS-D3 doctoral scholarship and the study was funded by the Prostate Cancer Canada. 


\section{CHAPTER 5}

\section{POST-IMPLANT DOSIMETRY OF PERMANENT PROSTATE BRACHYTHERAPY: COMPARISON OF QSM-BASED MRI-ONLY AND CT-MRI FUSION WORKFLOWS}

Under the second review by the International Journal of Radiation Oncology, Biology and

Physics: Nosrati R, Wronski M, Tseng C, Chung H, Pejović-Milić A, Morton G, Stanisz G. "Post-implant dosimetry of permanent prostate brachytherapy: comparison of QSM-based MRIonly and CT-MRI fusion workflows". International Journal of Radiation Oncology, Biology and Physics, Submision ID: ROB-D-19-01030 (2019).

Authors' contributions: R. Nosrati designed and carried out the experiments, designed and performed the post-processing algorithm and drafted the manuscript. M. Wronski helped with CT scans for validation of the MR-derived parameters and critically reviewed the manuscript. G.

Morton, C. Tseng and H. Chung referred their patients for clinical evaluation, provided advice throughout the course of this work and assessed the manuscript critically. A. Pejovic-Milic assessed the manuscript critically and provided critical feedback. G. Stanisz financed this project and provided advice throughout the project as well as critical feedback to the content, experimental design and the manuscript. 


\subsection{Abstract}

\subsubsection{Purpose}

The current MRI-CT fusion-based workflow for post-implant dosimetry of Low-Dose-Rate (LDR) prostate brachytherapy takes advantage of the superior soft tissue contrast of MRI but still relies on CT for seed visualization and detection. Recently an MR-only workflow has been proposed that employs standard MR sequences and visualize conventional implanted seed with positive contrast solely through MR post-processing. In this work, the novel MR-only based workflow is compared to the clinical CT-MRI fusion approach.

\subsubsection{Materials and Methods}

Twenty-four prostate patients with a total of 1775 implanted LDR seeds were scanned using a 3D multi-echo gradient echo sequence on a 3T MR scanner within 30 days following implantation. Quantitative Susceptibility Mapping (QSM) was used for seed visualization. Seeds

were automatically segmented and localized on the QSM using Convolutional Neural Network (CNN) and k-means clustering, respectively. To assess the MR-only seed localization error, CT and MR-derived seed positions were co-registered, and ultimately, the resulting Dose-VolumeHistograms (DVH) were compared.

\subsubsection{Results}

The proposed pipeline for MR-based seed visualization, segmentation and localization generated comparable results to the CT-MR registration approach. The accuracy of the MRI-only based seed identification was $99.1 \%$. Following a rigid registration between the MR and CTderived seed centroids the average localization errors was $0.8 \pm 0.8 \mathrm{~mm}$. The average prostate $\mathrm{D}_{90}$, 
$\mathrm{V}_{100}, \mathrm{~V}_{150}$, and $\mathrm{V}_{200}$ for MRI-only and CT-MR fusion based dosimetry were $114.3 \%$ vs. $113.9 \%$, $95.1 \%$ vs. $95.3 \%, 54.5 \%$ vs. $55.0 \%$ and $22.9 \%$ vs. $23.2 \%$ respectively. No significant differences were observed in 3D seed positions and dosimetric parameters between MR-only and CT-MR fusion-based workflows.

\subsubsection{Conclusion}

The MRI-only LDR post-implant dosimetry is reliable and has an excellent potential to eliminate the need for CT-based seed identification.

\subsection{Introduction}

Permanent implantation of low-dose-rate (LDR) brachytherapy seeds is a well-established treatment modality for patients with localized prostate cancer. The quality of the implant is assessed within 30 days following implantation through post-implant dosimetry. The standard recommended procedure for post-implant dosimetry is based on computed tomography (CT). CT provides excellent seed visualization and localization; however, due to poor soft tissue contrast hence challenging prostate volume identification, it leads to significant interobserver variabilities(Dubois et al., 1998; W. R. Lee et al., 2002). Recently magnetic resonance imaging (MRI) has been introduced to the LDR post-implant dosimetry workflow to benefit from its superior soft-tissue contrast(Ash et al., 2000; Davis et al., 2012; Segedin \& Petric, 2016). However, due to the lack of NMR signal from the seeds, they appear as dark voids on MR images, and seed localization still relies on CT. The CT-MRI fusion workflow has been recommended by the recent American Brachytherapy Society (ABS) guideline to take advantage of both imaging modalities 
by CT-based seed localization with MRI-based target delineation(Davis et al., 2012). It has been shown that the uncertainties associated with the MR-CT fusion may lead to up to $16 \%$ deviation in dose to $90 \%$ of the prostate (D90)(De Brabandere, Hoskin, Haustermans, Van Den Heuvel, et al., 2012; Dehghan et al., 2016; Kunogi et al., 2015; Polo et al., 2004b).

There are very limited clinical studies evaluating the feasibility of MRI-based brachytherapy seed detection and localization. Application of contrast-enhanced MR sequences in seed identification has been studied in(Buch et al., 2014; Ohashi et al., 2012a; Tanaka et al., 2006b) however, they have been limited by inconsistent MR sequence parameters, unreliable performance for extraprostatic or non-spaced seed identification. Zijlstra et al. have studied the feasibility of a template matching algorithm that uses the simulated magnetic field distortions around the seeds as the template to match with those in patients; although the proposed method has acceptable performance in detection of spaced seeds (average error of $0.8 \pm 0.4 \mathrm{~mm}$ ), one third of non-spaced or clumped seeds were not correctly identified(Zijlstra et al., 2017). Martin et al. have compared the MRI-only LDR post-implant dosimetry (using stranded seeds with cobalt-dichloride-N-acetyl cysteine (C4) MR-markers as spacers) to the standard MR-CT fusion-based approach(T. Y. Lim, Stafford, Kudchadker, Sankaranarayanapillai, Ibbott, Rao, Martirosyan, Frank, et al., 2014; Martin, Pugh, et al., 2017). They have demonstrated that MRI-only dosimetry using C4 MR markers is feasible and accurate, however, there are some limitations associated with $\mathrm{C} 4$ application such as higher cost compared with conventional seeds, the necessity of endorectal coil for marker visualization, and non-applicability to plans with non-spaced or loose seeds(Martin, Pugh, et al., 2017). It should be considered that in all the aforementioned approaches the implanted seeds still appear as dark voids making it challenging to be differentiated from other voids such as calcifications and small blood vessels especially in the case of extraprostatic seeds. MRI-based 
positive contrast seed visualization would make it easier for the physicist or the radiation oncologist to review and approve the post-plan after running the automated seed finder.

Recently Nosrati et al. have proposed and validated an MRI-only workflow based solely on MR post-processing algorithms, which generates high quality positive contrast for conventional brachytherapy seeds(Nosrati et al., 2018). The proposed algorithm exploits the strong paramagnetic properties of the titanium seeds in contrast to the diamagnetic biological tissues (e.g. prostate, calcifications, etc.) and use magnetic susceptibility to visualize seeds with positive contrast. The present study aimed at comparing the MRI-only workflow proposed in(Nosrati et al., 2018) to the standard CT-MRI fusion-based approach in terms of seed visualization, detection and dosimetric outcomes.

\subsection{Materials and Methods}

\subsubsection{Patients}

The study was conducted in accordance with the International Conference on Harmonization Good Clinical Practice E6 (ICH-GCP), Declaration of Helsinki principals as well as the set forth in The Belmont report. Institutional Research Ethical Board approval was obtained and all subjects provided written informed consent.

Twenty-four patients with low to intermediate-risk prostate cancer who were treated with LDR brachytherapy (as monotherapy) participated in this study. All patients were implanted with stranded I-125 seeds (IsoAid Advantage ${ }^{\mathrm{TM}}$ ). The patient characteristics and treatment planning parameters are shown in supplementary Table 5.1. Within one month following implantation, all patients underwent a pelvic CT scan and prostate MRI (approximately 30 minutes apart on the 
same day) to perform CT-MRI fusion-based post-implant dosimetry. Image fusion, post-planning and dosimetric analysis were performed on MIM Symphony brachytherapy planning program (MIM Software Inc, Cleveland, $\mathrm{OH}$ ).

\subsubsection{MRI and CT imaging protocols}

One month MRI scan was acquired on a 3T MRI scanner (Philips Achieva ${ }^{\mathrm{TM}}$ ) with a 16channel torso coil (Sense XL Torso). The standard of care MRI sequences for post-implant dosimetry included an axial 3-dimensional (3D) T1-weighted, an axial T2-weighted and diffusion weighted imaging (DWI). In addition to the standard of care, patients were scanned with a 3D fast spoiled multi-echo gradient recalled echo (GRE) sequence with fat suppression (SPIR) MR sequence. The GRE MRI sequence parameters were: echo time $=2.3 \mathrm{~ms}$, repetition time $=10 \mathrm{~ms}$, number-of-echoes $=3$; echo spacing $=2.3 \mathrm{~ms}$, flip angle $=15^{\circ} ; \mathrm{FOV}=225 \times 225 \times 100 \mathrm{~mm}^{3}$; resolution $=1 \times 1 \times 1.5 \mathrm{~mm}^{3}$, and bandwidth $=868 \mathrm{~Hz} /$ pixel. The MR scan time was approximately 10 minutes.

The patients were also CT scanned (Philips Brilliance Big Bore ${ }^{\mathrm{TM}}$ ) with the standard pelvic CT parameters: $120 \mathrm{kVp}, 400 \mathrm{mAs}$, and $3 \mathrm{~mm}$ slice thickness (reconstructed at $1.5 \mathrm{~mm}$ slice thickness).

Table 5.1; Patient characteristics and brachytherapy treatment planning parameters

\begin{tabular}{lc}
\hline Age (years), Mean \pm SD & $66.9 \pm 7.5$ \\
\hline Prostate volume (cc), Mean \pm SD & $39.3 \pm 19.2$ \\
\hline Rectum volume (cc), Mean \pm SD & $30.6 \pm 16.3$ \\
PSA level (ng/ml), Mean \pm SD & $7.9 \pm 3.2$ \\
\hline
\end{tabular}




\begin{tabular}{ll}
\hline Prescribed dose $(\mathrm{Gy})$ & 145 \\
\hline Number of implanted seeds, Mean \pm SD & $74.4 \pm 16.8$ \\
\hline Number of double loadings, Mean \pm SD & $2.4 \pm 1.7$ \\
\hline Clinical tumor stage range & $\mathrm{T} 1 \mathrm{c}-\mathrm{T} 2 \mathrm{~b}$ \\
\hline
\end{tabular}

\subsubsection{MR-based seed visualization}

The MR magnitude and phase images acquired by the GRE sequence were post-processed with MATLAB software (version R2018b, The Mathworks, Natwick, MA). The MR postprocessing pipeline included the following main two steps: (1) seed-induced MR distortion correction and edge enhancement(Jeffrey A Fessler \& Xed, 1997; A Matakos et al., 2014; Antonios Matakos \& Fessler, 2010); (2) Quantitative Susceptibility Mapping (QSM) based on Morphology Enabled Dipole Inversion with automated zero referencing (MEDI+0)(J. Liu et al., 2012b; T. Liu, Khalidov, et al., 2011; Z. Liu et al., 2018; Nosrati et al., 2018). The prostate tissue and the obturator internus were considered as the reference tissues and were automatically segmented by thresholding the GRE magnitude images at $50 \%$ of the maximum. The details of the positive contrast seed visualization algorithm are shown in Figure 1 (seed visualization block). 


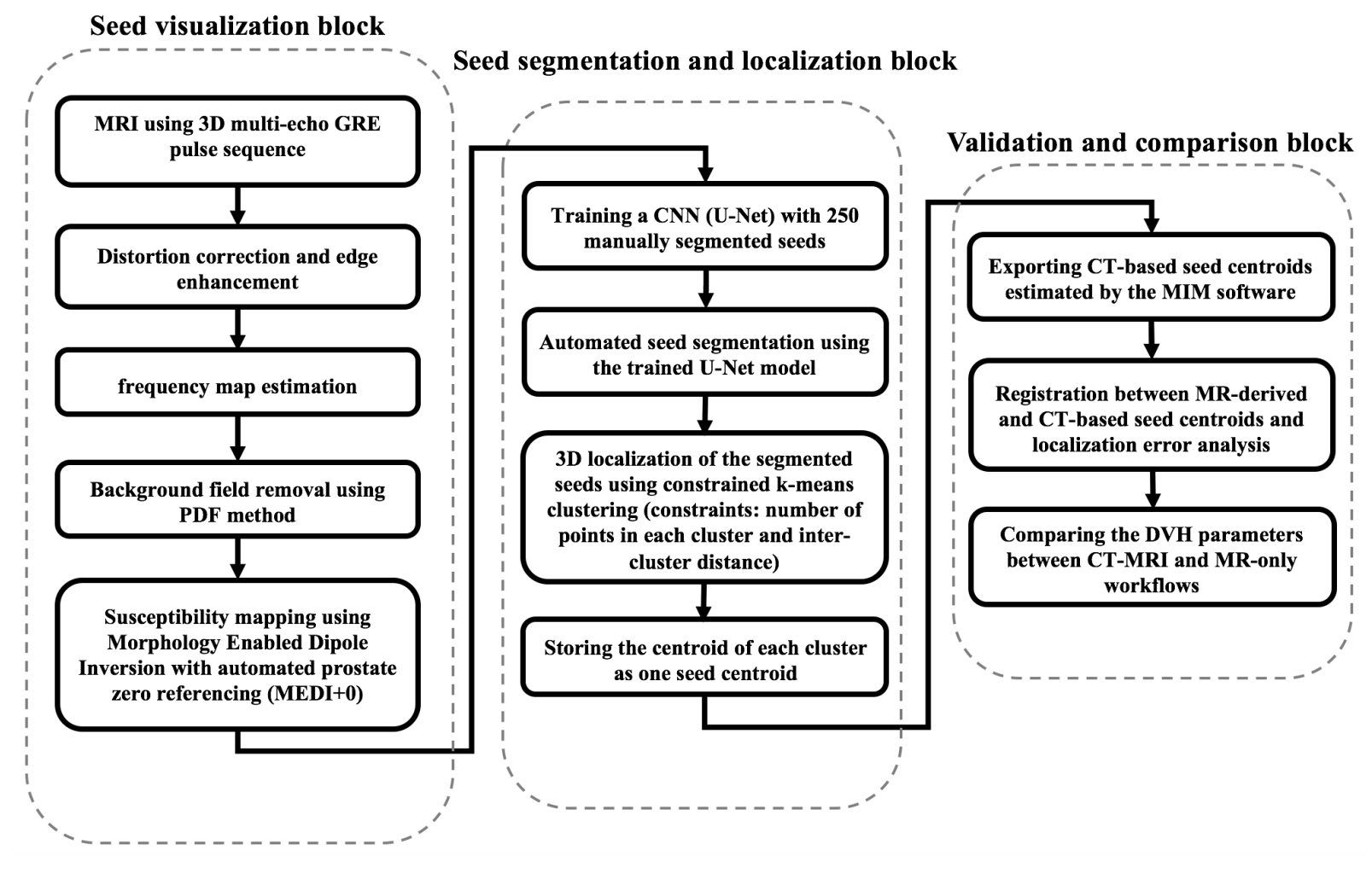

Figure 5.1; MRI post-processing pipeline

\subsubsection{MR-only seed localization}

The seed localization algorithm was modified compared to the method proposed previosuly(Nosrati et al., 2018). In almost half of the patients, the rectum was filled with gas, which resulted in the relatively large hyperintense region on QSM within rectum near the prostate. On the estimated susceptibility maps, the intensity of large volumes of gas in rectum at some voxels was close to that of seeds; thus, the gas-filled rectum was not completely removed by thresholding the QSM. Therefore, in this study, the seed identification on the magnetic susceptibility map in the presence of gas-filled rectum was accomplished by automated seed segmentation using supervised machine learning. Seed segmentation on QSM was performed using a deep, fully convolutional Neural Network (CNN). The 2D U-Net(Ronneberger, Fischer, 
\& Brox, 2015) model shown in Figure 2 was trained and used for automated seed segmentation. The U-Net architecture has symmetric contraction and expansion paths; the contraction path consisted of ten repeated $3 \times 3$ convolution operations in 5 steps (unpadded convolution); each convolution was followed by a Rectified Linear Unit (ReLU) activation function and batch normalization, and after each step a down-sampling ( $2 \times 2$ max pooling) was performed that halves the size of each feature channel. The expansion path included eight $3 \times 3$ convolution operations in four steps, each convolution was followed by a ReLU activation function and batch normalization; every step of the expansive path had a "transpose convolution" which was an up-sampling followed by a $2 \times 2$ convolution to halve the number of feature channels but double the size of the feature map(Ronneberger et al., 2015). To train the U-Net model, seeds were manually segmented on the QSM images of three random patients and 60 sets of labelled images were generated. The model was trained with a total of 280 labelled seeds and the accuracy of the segmentation when tested on the rest of the QSM images was 0.96 . The training time was 110 minutes, and the testing was about 1 second per image. The U-Net model was implemented in Python (Spyder, Python 3.6) using the open-source Keras package(Chollet, 2015).

Following seed segmentation, the $3 \mathrm{D}$ coordinates of seed centroids were identified using constrained k-means clustering based on the spatial distribution of the positive contrast voxels(Ester et al., 1996; Park \& Jun, 2009; Sander et al., 1998). The "constrained" clustering was used to ensure proper identification of non-spaced (clumped) seeds thus localization of the seeds was constrained by the number of voxels (points) in each seed (cluster) and the interseed (intercluster) distances. 


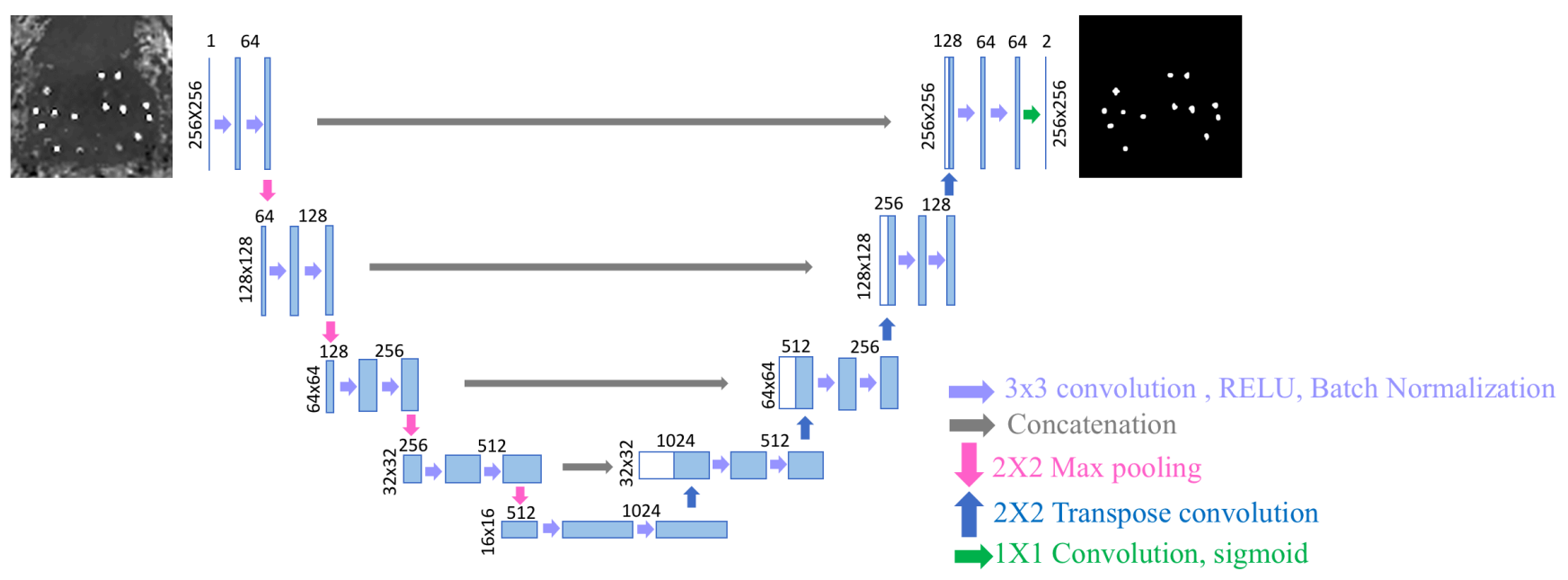

Figure 5.2.; U-Net model architecture; blue boxes represent multi-channel feature maps. The size of the input and output labeled images were $256 \times 256$. The number of the channels in each layer are shown by a number on top of each box.

\subsubsection{CT-MRI versus MR-only seed localization and dosimetry}

The positions of the brachytherapy seeds were identified on CT images with MIM Symphony. The 3D position of the seeds determined through the proposed MR-only based workflow were compared to the CT-based positions identified by MIM software. To evaluate the spatial accuracy of the MR-only seed localization, the 3D coordinates of the MR-based seed centroids were transferred into the CT-based coordinates by rigid registration. Each seed centroid identified on QSM was rigidly transformed and assigned to the nearest seed centroid on CT using the Iterative Closest Point (ICP) algorithm(Kjer \& Wilm, 2010). The 3D distance between each pair of centroids (after registration) was considered as the error of the MR-based seed localization. In addition, to assess the agreement between the MR and CT-based seed positions the BlandAltman analysis with 95\% confidence interval (CI) was performed. 
For dosimetric analysis of the MR-only and CT-MR fusion approaches, the same sets of contours were used which were made by an experienced radiation oncologist. Prostate was contoured on T2-weighted MRI however, due to rectum deformations between the CT and MRI scans (mainly due to the rectal filling and gas content), the rectum was contoured on CT images. The overall dose distribution was calculated using the CT-based seed positions, and cumulative Dose Volume Histogram (DVH) curves were stored for comparison. Then the transformed (by applying translation and rotation) MR-derived seed centroids were imported into MIM, and the corresponding DVH curves were re-calculated and saved for analysis. The dose to $90 \%$ of the prostate volume $\left(\mathrm{D}_{90}\right)$, the prostate volume that receives $100 \%, 150 \%$ and $200 \%$ of the prescribed dose $\left(\mathrm{V}_{100}, \mathrm{~V}_{150}, \mathrm{~V}_{200}\right.$ respectively), and the dose to $2 \mathrm{cc}$ of the rectum $\left(\mathrm{D}_{2 \mathrm{cc}}\right)$ were compared between the two methods.

Two-tailed paired sample t-test was used to evaluate the significance of the difference between CT- and MRI-derived seed positions and dosimetric parameters. Statistical analysis was performed using the SPSS software (IBM, Armonk, NY).

\subsection{Results}

\subsubsection{MR-based Positive Contrast Seed Visualization}

Out of twenty-four recruited patients, one patient did not undergo MRI due to previous metal injury to the eye, and three patients were excluded from the analysis, two because of severe motion artifacts and one due to abnormally large rectum volume (filled with gas) that resulted in signal loss at the prostate-rectum boundary. The total number of seeds in the twenty patients who were included in the analysis was 1563 out of which 1555 seeds (99.5\%) were visualized with excellent positive contrast on the QSM. In total, QSM algorithm failed to correctly reconstruct eight seeds; 
all those seeds were very close $(<1 \mathrm{~cm})$ to the edges of the imaging FOV in superior-inferior direction. Figure 5.3 shows an axial slice of T2-weighted MRI and the Maximum Intensity Projection (MIP) of 5 mid-slices of the CT and QSM of illustrative five patients. Visually there was an excellent agreement between CT and QSM in terms of seed identification. There were on average, 2.4 double-loaded seeds in each patient and all were correctly visualized. An example axial, sagittal and coronal cross-section of the QSM with one double loading as well as extraprostatic seed is shown in Figure 5.4. The performance of the algorithm for positive contrast visualization of the seeds was similar between intraprostatic and extraprostatic seeds (the arrow in Figure 5.4a). As shown by arrows in Figure 5.3 large volumes of gas in rectum showed up with positive contrast on QSM and were not completely removed by thresholding the QSM thus automated seed segmentation was performed by the proposed CNN algorithm. 

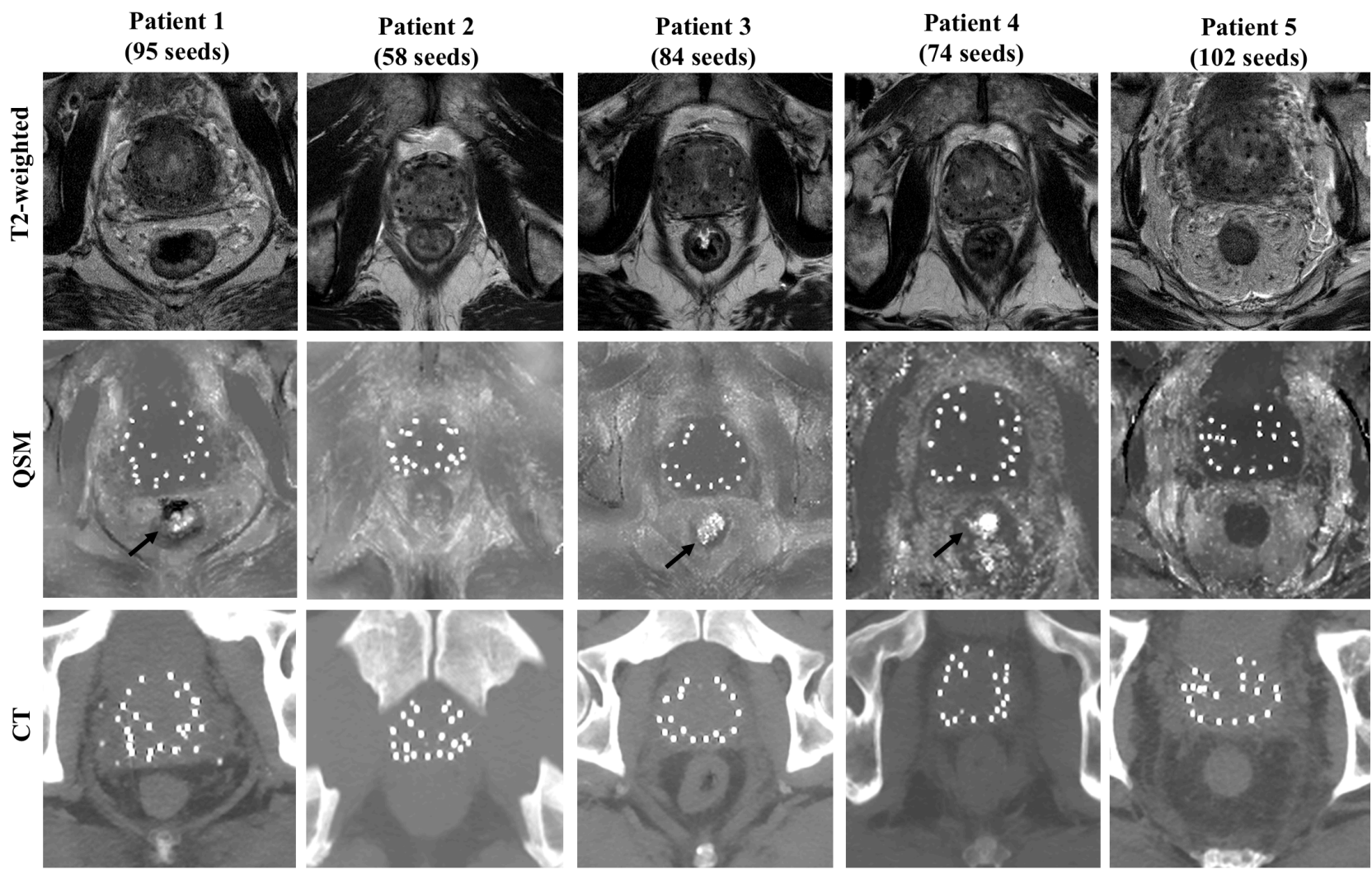

Figure 5.3; axial T2-wighted MR, and MIP reconstruction (5 mid-slices) of reconstructed QSM and CT images of 5 patients
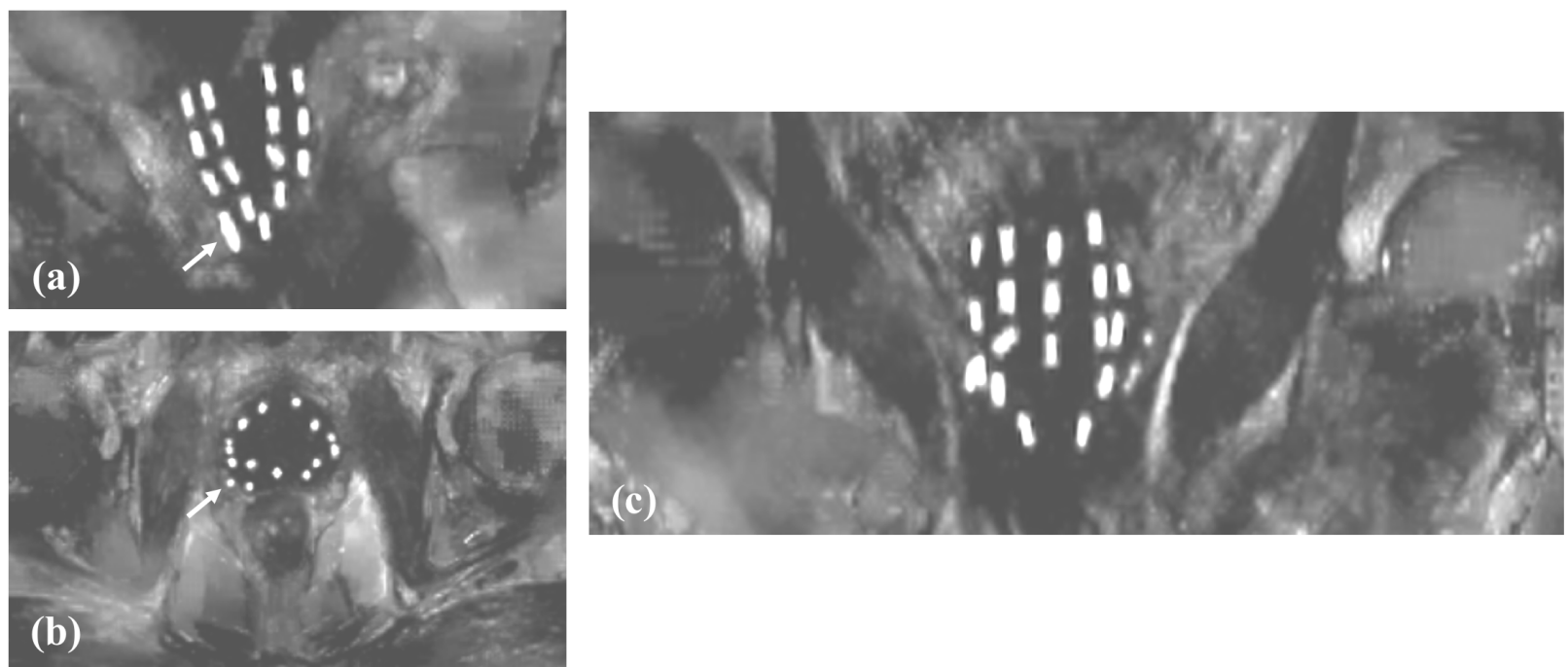

Figure 5.4; Example (a) sagittal, (b) axial and (c) coronal slices of the reconstructed QSM for one patient. The arrow in (a) shows an example of non-spaced seeds (double loading). 


\subsubsection{Seed Localization and Dosimetric Analysis}

Figure 5.5 shows three examples of seed segmentation results using the trained U-Net model. The presence of a gas-filled rectum with high susceptibility is visible on the first example of QSM images. However, the trained network correctly excluded rectum from the segmentation and only seeds were segmented. An example comparison between the registered MR-derived and CTderived seed centroids are shown in Figure 5.6a. Figure 5.6b shows the Bland-Altman analysis for the same patient; the Bland-Altman analysis revealed a very small bias of $-0.003 \mathrm{~mm}$ with narrow $95 \%$ limits of agreement of [-1.2 +1.2$] \mathrm{mm}$.

The proposed seed identification algorithm correctly identified 1549 out of 1563 seeds $(99.1 \%$ detection accuracy). Out of the fourteen mis-detected seeds, eight were not visualized with positive contrast due to proximity to the superior-inferior edges of the FOV and six were clumped seeds. The overall average error of the MR-only seed localization was $0.8 \pm 0.8 \mathrm{~mm}$ (excluding the 10 mis-detected seeds). The mean difference between MR and CT-based seed positions in superiorinferior $(\mathrm{Z})$, anterior-posterior $(\mathrm{X})$ and right-left $(\mathrm{Y})$ directions were $0.7 \pm 0.4 \mathrm{~mm}, 0.5 \pm 0.7$ and $0.8 \pm 0.5$ respectively. No significant difference was found between the MR-derived and CTderived seed centroids $(p>0.56)$.

Figure 5.6c-d show an example of post-implant dosimetry results using both CT and MR-based seed positions in one patient. In both methods, prostate and rectum were contoured on T2-weighted MRI and CT, respectively. The average D90 for prostate in MR-only and MR-CT fusion workflows were $165.5 \pm 18.1 \mathrm{~Gy}$ and $165.2 \pm 17.2 \mathrm{~Gy}$ respectively. The mean prostate $\mathrm{V}_{100}, \mathrm{~V}_{150}$ and $\mathrm{V}_{200}$ using MR-only and MR-CT fusion workflows were $95.1 \pm 3.7 \%$ vs. $95.3 \pm 3.8 \%, 54.5 \pm$ $14.5 \%$ vs. $55.0 \pm 13.2 \%$ and $22.9 \pm 6.7 \%$ vs. $23.2 \pm 6.8 \%$ respectively. The average $\mathrm{D}_{2 \text { cc,rectum }}$ with MR-only pipeline versus MR-CT fusion were $92.6 \pm 22.7 \%$ and $91.6 \pm 22.6 \%$ respectively. There 
was no significant difference between the dosimetric parameters between the two methods $(p>$ $0.3)$.

The average bias of the Bland-Altman analysis for all patients was +0.05 , and less than $5 \%$ of the seeds were outside the $95 \%$ limits of agreement.
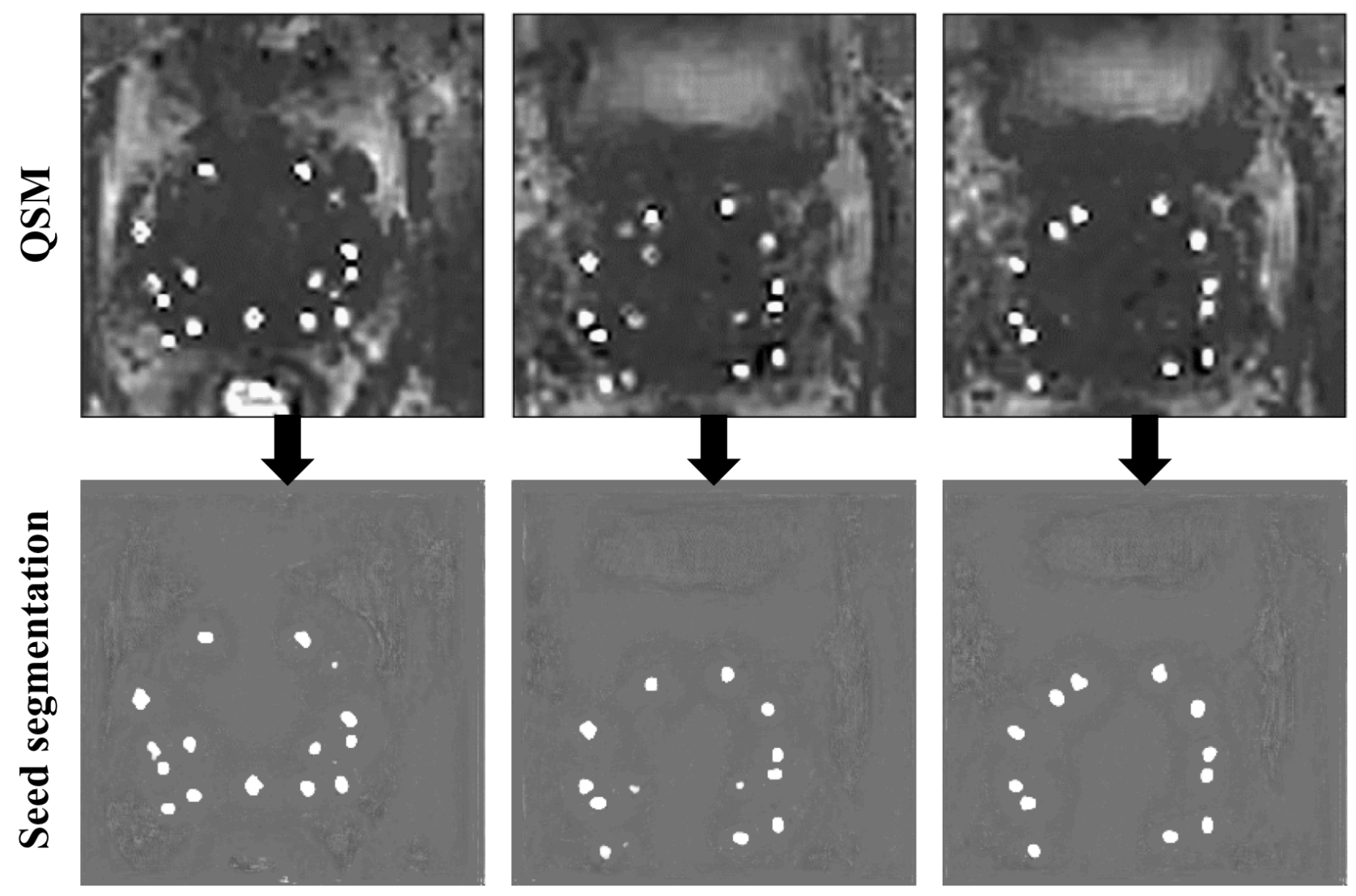

Figure 5.5; Some example automated seed segmentation results on QSM using the trained U-Net model. The top left QSM image shows high susceptibility area in the rectum due to gas (with similar susceptibility values to that of seeds) and the trained model successful 

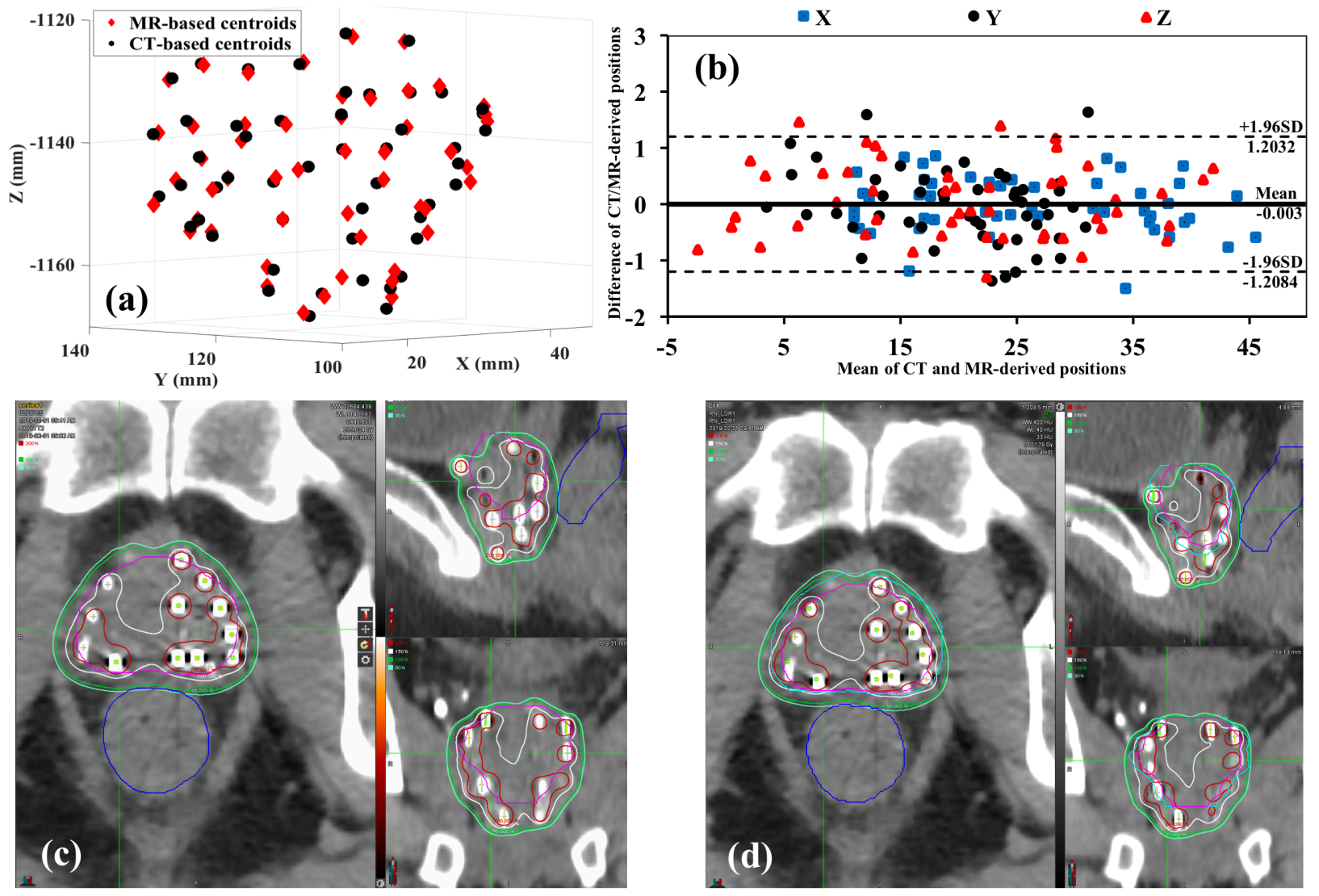

Figure 5.6; Seed localization and dosimetric analysis in one example patient. (a) 3D representation of CT and MR-derived seeds centroids; (b) the Bland-Altman plot for seed detection differences in X, Y and Z directions with a small bias of $-\mathbf{- 0 . 0 0 3}$ and $95 \%$ limits of agreement of $[-1.2+1.2] \mathrm{mm}$; (c) axial, sagittal and coronal CT images with identified seed centroids and isodose lines calculated based on CT and (d) axial, sagittal and coronal CT images with identified seed centroids and isodose lines calculated based on MRI (QSM). Prostate contouring was performed on T2-weighted MRI following CT-MRI fusion and the same set of contours were used for dosimetry.

\subsection{Discussion and Conclusions}

Traditionally post-implant dosimetry of permanent seed brachytherapy has been based on CT. However, due to the poor soft tissue contrast of $\mathrm{CT}$, which leads to significant interobserver variabilities, clinics have been encouraged to implement MR-CT fusion-based workflow to take 
advantage of the superior soft tissue contrast of MRI. Currently, permanent brachytherapy postimplant dosimetry strongly relies on $\mathrm{CT}$ for excellent positive contrast seed visualization, which is critical for dosimetry. In addition to the extra cost and logistics involved with MR-CT, the image fusion process is a potential source of error. Brabandere et al.(De Brabandere, Hoskin, Haustermans, Van den Heuvel, \& Siebert, 2012) showed that CT-MR fusion, especially CT and T2-weighted MR fusion resulted in up to $16 \%$ discrepancies in D90 mainly because fusion landmarks which are typically seeds are hardly visible on T2-weighted images. Previous studies on MRI-only post-implant dosimetry using contrast-enhanced T1-weighted images had poor results on the detection of seeds at the boundaries of the prostate and extraprostatic seeds, therefore, were not deemed clinically reliable(Bloch et al., 2007; Ohashi et al., 2012a). Frank et al.(Martin, Pugh, et al., 2017) have reported excellent performance of an MRI-only workflow using C4 MR markers with the application of endorectal coil (without any loose or clumped seeds). In the present work, we compared the seed visualization, localization and dosimetry between a novel MRI-only workflow vs. the standard CT-MRI fusion-based approach. The investigated pipeline showed $99.1 \%$ accuracy in MR-based seed identification; $0.5 \%$ of the seeds were misidentified (false negative) due to extreme proximity to the superior-inferior edges of the FOV and $0.4 \%$ of the seeds were not detected due to seed clumping. To ensure efficient seed visualization through QSM, the superior-inferior borders of the FOV should be defined at least $2 \mathrm{~cm}$ away from the prostate boundaries in that direction. Although clumped seeds could be manually identified on QSM, the proposed automated seed identification algorithm failed to correctly detect about $8 \%$ of the clumped seeds; further improvement of the seed identification algorithm, may potentially solve the challenge of clumped seed identification. A submillimeter average difference was observed between MR and CT-derived seed positions, which may be partially due to the small prostate 
deformations between the MR and CT scans which may have caused small discrepancies between relative seed positions between the two methods. One potential solution to better evaluate the MRbased seed identification would be to perform a deformable registration between the MR and CTbased seed positions (rather than rigid registration which was applied in this work) to account for prostate deformations as well as the whole volume translation and rotation. The DVH analysis showed that the minor differences in seed positions between MRI-only and MR-CT fusion, did not result in any significant difference in DVH parameters; this agreed with a previous study by Su et al.(Su et al., 2007) on minimal sensitivity of prostate dosimetric parameters to the seed localization accuracy.

One of the benefits of the MR-only approach in this study in comparison with the previous works was relying only on image post-processing and being applicable to conventional brachytherapy seeds. Unlike all mentioned previous clinical works, it generated distinctive positive contrast at the exact location of the seeds. Also, previous works have shown that the QSM can easily differentiate between paramagnetic brachytherapy seeds and diamagnetic prostatic calcifications(Nosrati et al., 2018, 2019).

In this study, we excluded three out of twenty-three scanned patients from the analysis due to motion artifacts and very large gas-filled rectum. The motion artifact was mainly in anteriorposterior direction (readout direction) and was more severe near the prostate apex. The possible sources of prostate motion are patient movement, breathing or rectal contractions. Reducing the scan time and preferably running the GRE sequence prior to the standard of care sequences will minimize the patient movements during the scan. Glucagon is commonly administered to prostate patients at many clinics (including the study by Frank et al.) prior to prostate MRI to suppress rectal movements and avoid image distortions(Martin, Pugh, et al., 2017). Administration of 
glucagon to reduce the motion of prostate adjacent structures, including rectum, bladder, small bowel may be considered. In addition, it is recommended to scan patients with empty bladder and empty rectum for optimal QSM results.

This study had a few limitations, including the small sample size and the extended MR scan time (by about 10 minutes) that made the scan more susceptible to motion artifact. In this work, the same set of contours were used for dosimetric analysis and the possible MR-CT fusion uncertainties were not taken into account; thus, assessment of the effect of any MR-CT fusion error in comparison with this MRI-only method could be a potential extension to this work.

In conclusion, the MRI-only post-implant dosimetry using QSM has shown to be feasible and accurate and offers a unique opportunity to replace the conventional CT-only or MR-CT fusionbased workflows. 


\section{CHAPTER 6}

\section{POTENTIAL APPLICATIONS OF THE QUANTITATIVE SUSCEPTIBILITY MAPPING (QSM) IN MR-GUIDED RADIATION THERAPY}

Published in the Journal Physics in Medicine and Biology: Nosrati R, Paudel M, Ravi A,

Pejović-Milić A, Morton G, Stanisz G. "Potential applications of the Quantitative Susceptibility Mapping (QSM) in MR-Guided Radiation Therapy”. Journal of Phys Med Biol. 2019 Jul $16 ; 64(14): 145013$

Authors' contributions: R. Nosrati designed and carried out the experiments, designed and performed the post-processing algorithm and drafted the manuscript. M. Paudel helped with CT scans for validation of the MR-derived parameters and critically reviewed the manuscript. G. Morton, provided advice throughout the course of this work and assessed the manuscript critically. A. Pejovic-Milic and A. Ravi assessed the manuscript critically and provided critical feedback. G. Stanisz financed this project and provided advice throughout the project and reviewed the manuscript critically. 


\section{1. $\quad$ Abstract}

\subsubsection{Background and Purpose}

Magnetic Resonance-Guided Radiation Therapy (MR-GRT) offers great potential to improve radiation treatment outcomes by providing more accurate and patient-tailored therapy. Despite superior soft tissue contrast in MRI, one of the challenges towards MRI-only workflows is that the process often requires some sort of "MR-invisible" metal-based devices. In this study, the feasibility of Quantitative Susceptibility Mapping (QSM) for visualization of some MR-invisible radiation therapy devices was studied.

\subsubsection{Materials and Methods}

Our recently proposed QSM-based algorithm for brachytherapy seed visualization was modified and the feasibility of the optimized algorithm for visualization of different devices including: brachytherapy seeds, plastic interstitial needles, CT-markers and obturators, and different types of fiducial markers in agar, prostate and meat phantoms were studied. All phantoms were scanned using 3T MR scanner with a 3D multi-echo gradient recalled echo (ME-GRE) pulse sequence. The QSM results in all phantoms were compared to CT images for spatial accuracy of the QSM.

\subsubsection{Results}

The applied post-processing algorithm was found to be insensitive to the seeds' type; also, presence of nearby calcifications had no effect on seed visualization. QSM successfully generated positive contrast for both types of investigated fiducial markers with high spatial accuracy compared to CT. Interstitial needles containing both aluminum-based CT-maker and titaniumbased obturators were accurately depicted on the QSM.

\subsubsection{Conclusion}

The proposed QSM-based technique relies on the standard MR pulse sequences and visualize the conventional MR-invisible metallic devices with CT-like positive contrast solely through post- 
processing. Upon in-vivo validation of the technique, QSM may have the potential to replace CT for an MR-only guided radiation therapy.

\subsection{Introduction}

Brachytherapy and external beam radiation therapy (EBRT) have a major role in the management of prostate and many gynecologic malignancies. Radiation treatment planning and delivery are image guided procedures which have evolved from 2D X-ray radiography to 3D ultrasound, computed tomography $(\mathrm{CT})$ and magnetic resonance imaging (MRI)(Kapur, Egger, Damato, Schmidt, \& Viswanathan, 2012). It is widely accepted that MRI offers superior soft tissue contrast in comparison to the other modalities, and therefore will be able to support more accurate/reproducible delineation of targets and organs at risk to deliver an accurate patienttailored radiation dose.

Despite poor soft tissue contrast, CT scan is an inevitable part of all of the radiation treatment planning and/or delivery processes which require temporary or permanent placement of paramagnetic objects within the target volume either for patient positioning or for accurate treatment delivery(Bowes, Crook, Araujo, \& Batchelar, 2013; Dehghan et al., 2016; Erickson, Albano, \& Gillin, 1996; Kulkarni, Hong, Kambadakone, \& Arellano, 2015; Kunogi et al., 2015). This includes but is not limited to the permanent low-dose-rate (LDR) brachytherapy seeds,

fiducial markers for patient positioning for both external beam radiotherapy (EBRT) and brachytherapy, and high-dose-rate (HDR) interstitial gynecological brachytherapy needles, obturators/catheters and biopsy needles.

These medical devices are mainly constructed with MR and bio compatible paramagnetic materials (such as titanium, gold and plastic) however, due to lack of hydrogen nuclei they do not 
generate MR signal hence are not MR visible and appear as diffused hypointense regions on conventional MR images. This makes them indifferentiable from other MR signal voids such as blood vessels, calcifications, and cavities. On the other hand, these MR-invisible materials have relatively high electron density thus excellent contrast on CT images. Currently, at many centers CT is used for visualization of MR-invisible materials followed by an MR-CT image registration(Dehghan et al., 2016; Polo et al., 2004a).

To eliminate the need for a CT scan towards the MRI-only workflows for MR-guided radiation therapy a few solutions have been proposed to create CT-like positive contrast for metallic implants such as fiducial markers, interstitial brachytherapy needles and obturators, and Low-Dose-Rate (LDR) brachytherapy seeds. The proposed methods include custom-made MR pulse sequences (such as IRON and co-RASOR)(H. de Leeuw et al., 2013; Hendrik de Leeuw et al., 2013a; Kuo et al., 2010b), use of MR markers [such as cobalt chloride, cobalt dichloride-Nacetyl cysteine (C4), $\mathrm{CuSO}_{4}$ solutions, ultrasound (US) gel](Haack, Nielsen, Lindegaard, Gelineck, \& Tanderup, 2009; T. Y. Lim, Stafford, Kudchadker, Sankaranarayanapillai, Ibbott, Rao, Martirosyan, Frank, et al., 2014; Perez-Calatayud et al., 2009; Tanderup, Viswanathan, Kirisits, \& Frank, 2014; Wills et al., 2010), and MR post-processing algorithms such as susceptibility gradient mapping (SGM) and quantitative susceptibility mapping (QSM)(Dahnke et al., 2008; Yi Wang \& Liu, 2015). In general, the most desired MR-based solution is the one which requires minimal cost and minimum alterations to the standard devices and protocols. The postprocessing-based techniques which are applicable to the clinically available devices, MR scanners and pulse sequences are the ideal solution to this problem.

We have recently proposed an MRI-based workflow for visualization and localization of permanent LDR brachytherapy seeds in phantoms(Nosrati et al., 2018). The proposed method 
utilizes clinical MR pulse sequences on $1.5 \mathrm{~T}$ and 3T MR scanners and uses the magnetic susceptibility as the source of contrast (between implanted seeds and the soft-tissue background) for seed visualization. In the present work, the method proposed in(Nosrati et al., 2018) is modified by introducing background tissue zero referencing during the Morphology-Enabled Dipole Inversion process $(\mathrm{MEDI}+0)$ to minimize the QSM reconstruction artifacts specially near the large susceptibility mismatch boundaries (around metal implants). In addition, the feasibility of the optimized algorithm for visualization of some MR-invisible radiation therapy devises including fiducial markers (gold and carbon-coated zirconium), HDR interstitial brachytherapy needles, HDR CT-marker, obturators and biopsy needles are investigated. Also, some limitations of previous works on LDR brachytherapy seeds including sensitivity of the QSM to the internal configuration of the seeds as well as presence of calcifications near the seeds are studied.

\subsection{Materials and Methods}

\subsubsection{Phantom preparation}

Three agar-based tissue mimicking phantoms consisting of 3\% agar, $0.29 \% \mathrm{NaCl}, 0.03 \%$ (by weight) and $22.2 \mu \mathrm{mol} / \mathrm{kg}$ of $\mathrm{NaN}_{3}$ (Sigma-Aldrich Canada Co.) were prepared(Hattori et al., 2013).

To investigate the sensitivity of our pervious method(Nosrati et al., 2018) to the type of seeds (manufacturer/radionuclide) and presence of calcifications nearby very closely spaced seeds, one IsoAid I-125 (IsoAid Advantage ${ }^{\mathrm{TM}}$, USA), two IsoSeed I-125 (125.S06, Eckert \& Ziegler BEBIG, USA) and two IsoAid Pd-103 (IsoAid Advantage ${ }^{\mathrm{TM}}$, USA) seeds were placed in the middle layer 
of an agar phantom at random orientations and two pieces $(4.5 \mathrm{~mm}$ and $8 \mathrm{~mm}$ long) of bone were placed very close to the seeds (1-2mm distance). As shown in Figure 6.2a, all seeds have similar external dimensions of $4.5 \mathrm{~mm} \times 0.8 \mathrm{~mm}$. The configuration of the seeds and bone in the agar phantom is shown in Figure 6.2b.

To assess the feasibility of the proposed method for visualization of the fiducial markers, two types of markers were studied: the carbon coated zirconium markers with $0.9 \mathrm{~mm} \times 5 \mathrm{~mm}$ dimensions (BiomarC PRO, Carbon Medical Technologies, USA) and the standard gold fiducial markers with dimensions of $1.2 \mathrm{~mm}$ x $3 \mathrm{~mm}$ (CIVCO Radiotherapy, USA). Three gold markers were placed $3.4 \mathrm{~cm}$ apart at random orientations in the middle layer of an agar phantom (Figure 6.3b) and three carbon markers were implanted into a realistic prostate phantom (CIRS Inc. 053L model) by an experienced radiation oncologist (Figure 6.4b).

The feasibility of the algorithm presented in Figure 6.1 was also studied for MRI-based visualization of HDR interstitial brachytherapy treatment planning. The performance of the QSM pipeline was assessed on positive contrast depiction of hollow plastic needles (ProGuide 6F, Elekta AB, Sweden), aluminum-based markers (CT marker, Elekta AB, Sweden), titanium-based obturators (ProGuide Obturator, Elekta AB, Sweden), and solid pure titanium rod (2 mm diameter) (Figure 6.5a). In the agar phantom two plastic needles with CT-markers, two plastic needles with ProGuide obturators and one pure titanium rod were inserted (Figure 6.5b). In addition, for a more realistic background medium, two hollow needles, one needle with CT marker and the other needle with titanium-based obturator were inserted into a beef meat phantom (Figure 6.5c).

The magnetic susceptibility and atomic number of the construction materials in the studied devices/phantoms are presented in Table 6.1. 
Table 6.1; Volume magnetic susceptibility and atomic number of the studied materials(Fleming \& Dewar, 1898; Haacke, Xu, Cheng, \& Reichenbach, 2004; Javad, Birgani, Seif, Chegeni, \& Bayatiani, 2012; Saito \& Sagara, 2017; Schenck, 1996; "Volume Magnetic Susceptibility for all the elements in the Periodic Table,” n.d.).

\begin{tabular}{lllll}
\hline Material & Relevant tissue/device & $\begin{array}{l}\text { Magnetic } \\
\text { properties }\end{array}$ & $\begin{array}{l}\text { Magnetic } \\
\text { susceptibility (ppm) }\end{array}$ & $\begin{array}{l}\text { Effective atomic } \\
\text { number }\end{array}$ \\
\hline Water & Soft tissue & Diamagnetic & -10 & $7.42^{*}$ \\
Calcium carbonate & Bone / calcification & Diamagnetic & -38.2 & $10^{*}$ \\
Hydroxyapatite & Bone / calcification & Diamagnetic & -14.8 & $16.2^{*}$ \\
Titanium & Seeds / ProGuide obturator & Paramagnetic & +180 & 22 \\
Silver & Seeds & Diamagnetic & -23 & 47 \\
Gold & Seeds / fiducial marker & Diamagnetic & -34 & 79 \\
Zirconium & BiomarC fiducial marker & Paramagnetic & +109 & 40 \\
Carbon & BiomarC fiducial marker & Diamagnetic & -14 & 6 \\
Polyethylene & Plastic needles & Diamagnetic & N/A & $4.6^{*}$ \\
Nickle & HDR CT-marker & Ferromagnetic & $+600 x 10^{6}$ & 28 \\
Polyimide & HDR CT-marker & Diamagnetic & -8 & $6.8^{*}$ \\
Aluminum & HDR CT-marker & Paramagnetic & +21 & 13 \\
Air & Hollow needles / seeds & Paramagnetic & +0.024 & $7.6^{*}$ \\
\hline & & & & 28 \\
\hline
\end{tabular}

*effective atomic number 


\subsubsection{Imaging protocols}

All phantoms were scanned with 3T MRI scanner (Philips Achieva ${ }^{\mathrm{TM}}$ ) using an 8-channel head coil array. A 3D multi echo gradient recalled echo (ME-GRE) sequence with the following parameters was applied: $\mathrm{TE}_{1} / \mathrm{TR}=2.3 / 10.3 \mathrm{~ms}$; number of echoes $=3$; echo spacing $=2.3 \mathrm{~ms}$; flip angle $=15^{\circ} ; \mathrm{FOV}=140 \times 140 \times 100 \mathrm{~mm}^{3}$; bandwidth $=868 \mathrm{~Hz} /$ pixel; resolution $=1 \times 1 \times 1 \mathrm{~mm}^{3}$. MR scan of the meat phantom was performed with fat saturation using Spectral Pre-saturation Inversion Recovery (SPIR) technique (Del Grande et al., 2014). The MR acquisition time varied between 3 - 5 minutes for different phantoms. To validate MR-based results all phantoms were also CT scanned (Philips Brilliance Big Bore ${ }^{\mathrm{TM}}$ ) with $120 \mathrm{kVp}, 400 \mathrm{mAs}$, and $1.5 \mathrm{~mm}$ slice thickness.

\subsubsection{MR image post-processing pipeline}

Magnitude and phase images from the GRE MR sequence were used for post-processing. Given the significant difference between the magnetic susceptibility of all investigated devices and soft tissue (Table 1), Quantitative Susceptibility Mapping (QSM) was performed to take advantage of the magnetic susceptibility as the main source of contrast. The general post-processing pipeline that was employed in all investigated applications is shown in Figure 6.1. The QSM method proposed in (Nosrati et al., 2018) was slightly modified for optimal results in different applications.

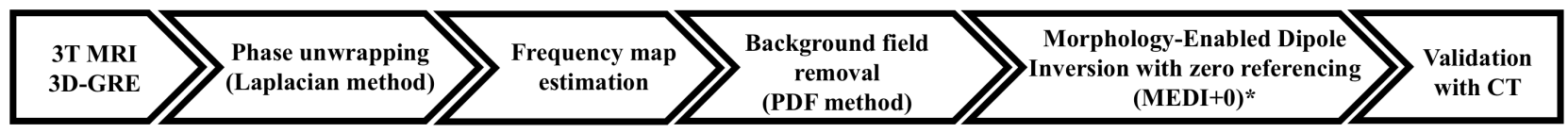

Figure 6.1; the MR image post-processing pipeline. 
The general QSM pipeline included the following steps: (1) phase unwrapping using Laplacian method (Schofield \& Zhu, 2003), (2) field map estimation using non-linear least square fitting (de Rochefort et al., 2008; Kressler et al., 2010; T. Liu et al., 2013), (3) background field estimation using Projection onto Dipole Field (PDF) method (T. Liu, Khalidov, et al., 2011) and subtraction from the total field map calculated in the previous step, (4) Morphology Enabled Dipole Inversion with background zero referencing (MEDI+0) (T. Liu, Liu, et al., 2011; Z. Liu et al., 2018). The main modification in the post-processing pipeline compared to the method proposed in (Nosrati et al., 2018) was the use of MEDI +0 technique for dipole inversion which enforces the uniformity in the background and reduce the susceptibility reconstruction artifacts at the susceptibility mismatch boundaries.

To apply the MEDI +0 method a binary mask was generated by thresholding the magnitude images to assign zero value to the pixels with signal voids and one to the background. The following minimization problem was solved for dipole inversion and susceptibility $\left(\chi_{r}\right)$ estimation:

$$
\chi_{r}=\operatorname{argmin}_{\chi_{r}}\left\|W\left[e^{i D_{r} \chi_{r}}-e^{i f_{r}}\right]\right\|_{2}^{2}+\lambda_{1}\left\|G_{M} G_{\chi}\right\|_{1}+\lambda_{2}\left\|M\left[\chi_{r}-\overline{\chi_{B}}\right]\right\|_{2}^{2}
$$

where $D_{r}$ is dipole kernel, $f_{r}$ is the local field map $W$ is a weighting matrix calculated using a binary mask normalized to the noise standard deviation which was tuned in each iteration (T. Liu et al., 2013); $G$ is the gradient operator; $M$ is a binary mask calculated by thresholding the magnitude images for zero referencing; $\overline{\chi_{B}}$ is the average estimated susceptibility within the 
segmented background; $\lambda_{1}$ and $\lambda_{2}$ are the regularization parameters which both were set to 100 . The L1 regularization term enforces the agreement between the edges in magnitude image and the edges in the estimated susceptibility map (J. Liu et al., 2012a; T. Liu, Liu, et al., 2011; Yi Wang \& Liu, 2015). The second L2 regularization term enforces susceptibility homogeneity within the segmented background. The L2-regularization of the background susceptibility is similar to the L1-regularization term of the gradients and is considered as an additional prior structural constraint that reduces artifacts at the susceptibility mismatch interface(Z. Liu et al., 2018). The MR image post-processing time varied between 2 - 4 minutes for QSM reconstruction of different phantoms. The Maximum Intensity Projection (MIP) of all image sets (QSM and CT) were reconstructed for $3 \mathrm{D}$ visualization and comparison.

\subsection{Results}

\subsubsection{Effect of seed type and nearby calcifications}

Figure 6.2 shows different structures of the LDR brachytherapy seeds that were studied along with the constructed phantom (middle layer) containing seeds and calcifications, MR magnitude/phase images, QSM and CT images of the phantom. Although each of the five seeds had a different orientation with respect to the static magnetic field $\left(\mathrm{B}_{0}\right)$ the size, shape and intensity of the reconstructed seeds on QSM were similar for all three types of seeds. All seeds and calcifications appeared as dark voids on magnitude images (Figure 6.2c) however, as shown in Figure $6.2 \mathrm{~d}$ the field distortions around the seeds are more pronounced around the seeds compare with bones (calcification). 
Figure 6.2e shows that on the reconstructed QSM, only paramagnetic seeds have positive contrast and diamagnetic calcifications (bones) have negative contrast. Considering the complex structural/chemical composition of the seeds and bones, the average estimated magnetic susceptibility values for seeds and bones presented in Table 2 were within a reasonable range in comparison with the expected values (Table 1).

The average length of the reconstructed seeds on QSM was $4.5 \pm 0.5 \mathrm{~mm}$ which is comparable to the actual length of the seeds.

(a)
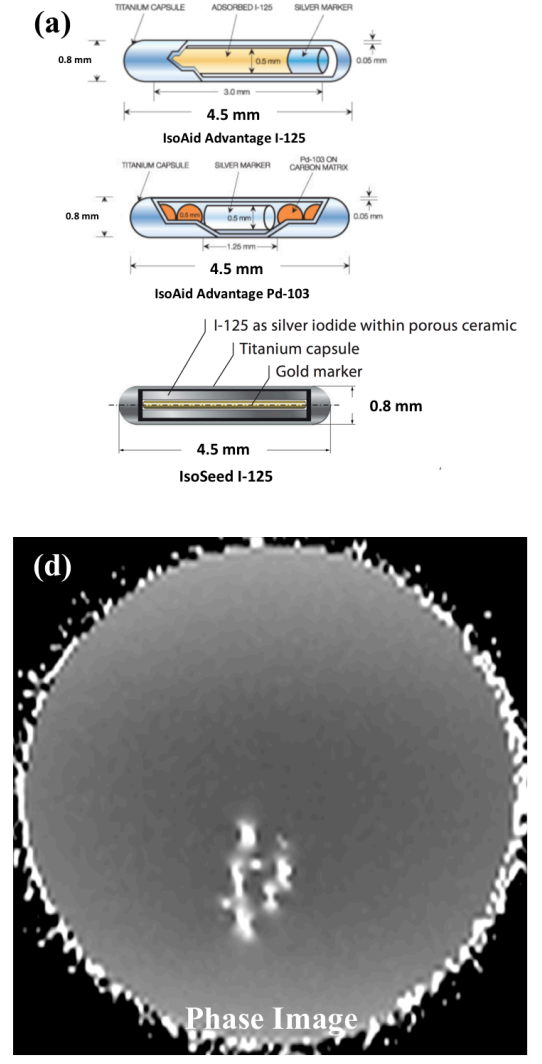
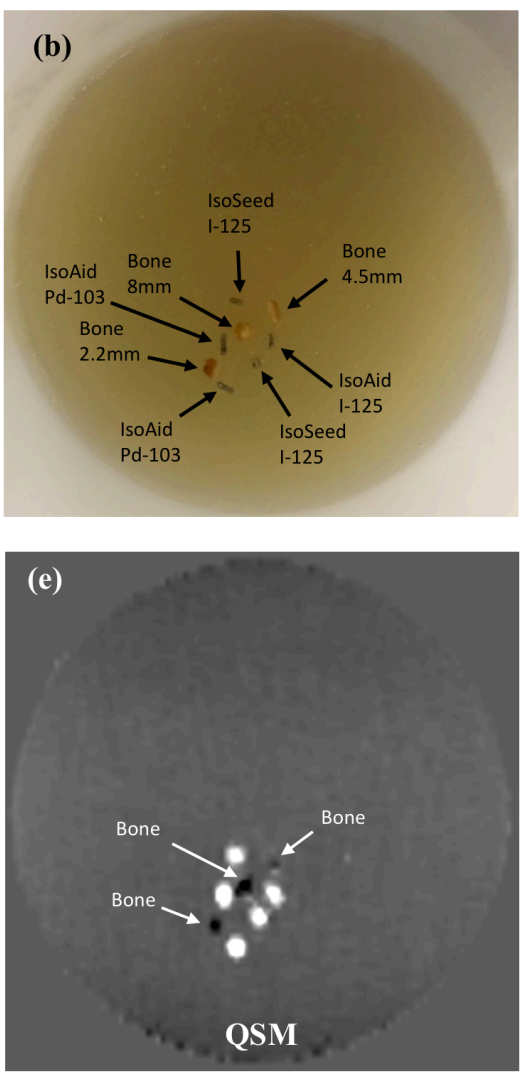
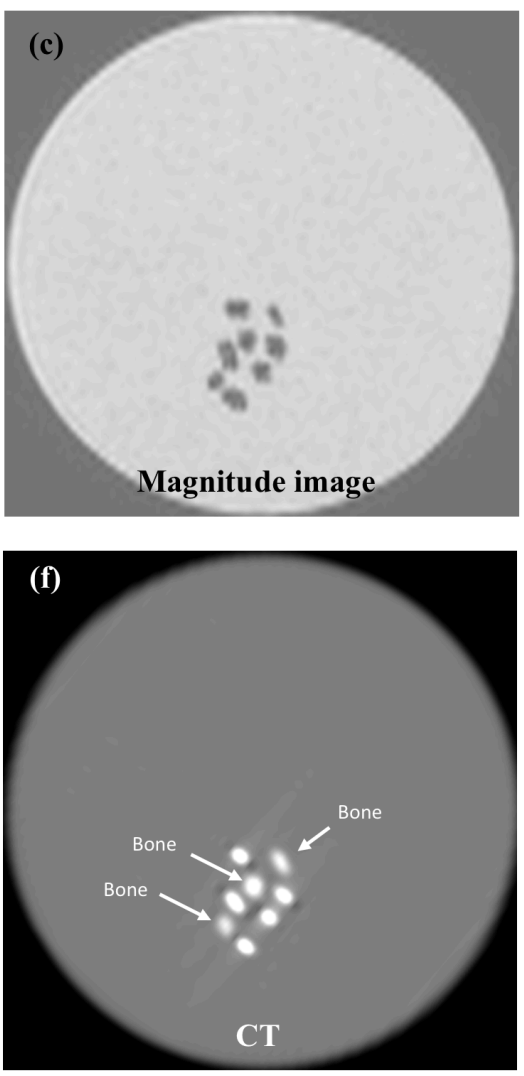

Figure 6.2; (a) internal configuration of the seeds that were studied [41,42]; (b) constructed agar-based phantom containing 5 seeds and 3 calcifications (bone); (c) MR magnitude image at shortest TE; (d) MR phase image at shortest TE; (e) An axial slice of the reconstructed QSM which contains seeds and calcifications; (f) the axial slice of $\mathrm{CT}$ that contains seeds and calcifications. 


\subsubsection{Fiducial markers}

Figure 6.3 shows the structure of the gold fiducial marker, constructed phantom and the corresponding axial slice of the MR magnitude and phase images, reconstructed susceptibility map (QSM), and CT image. As shown in Table 1, gold is a diamagnetic material similar to soft tissue however it's susceptibility is about 3.5 times less than that of soft tissue thus to visualize the gold fiducials with positive contrast, following susceptibility mapping the sign of the estimated QSM was inverted (multiplied by -1). As shown in Figure 6.3e the implanted gold markers were clearly depicted on inverted QSM with sufficient contrast. The QSM resulted in no artifacts around markers however, on CT (Figure 6.3f) streaking artifacts were present around them.

(a)
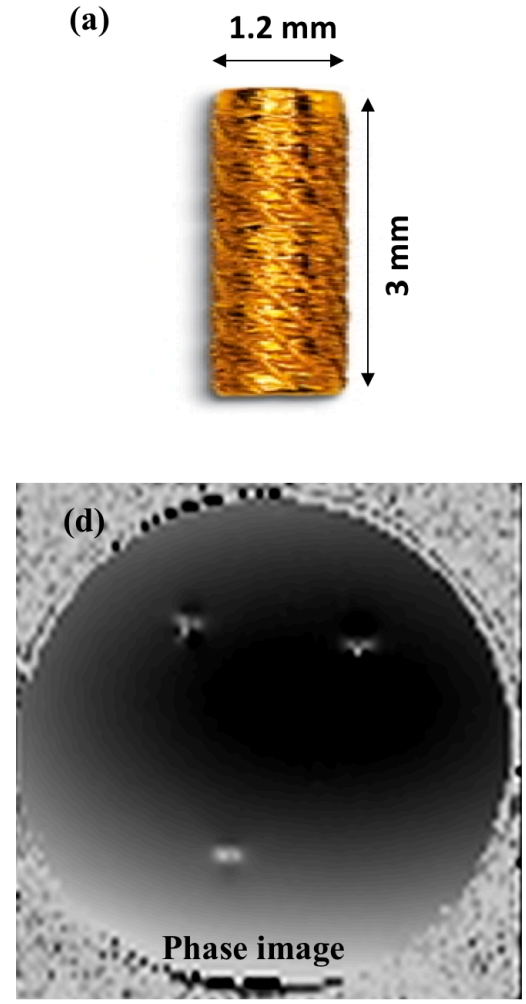

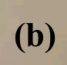

(b)
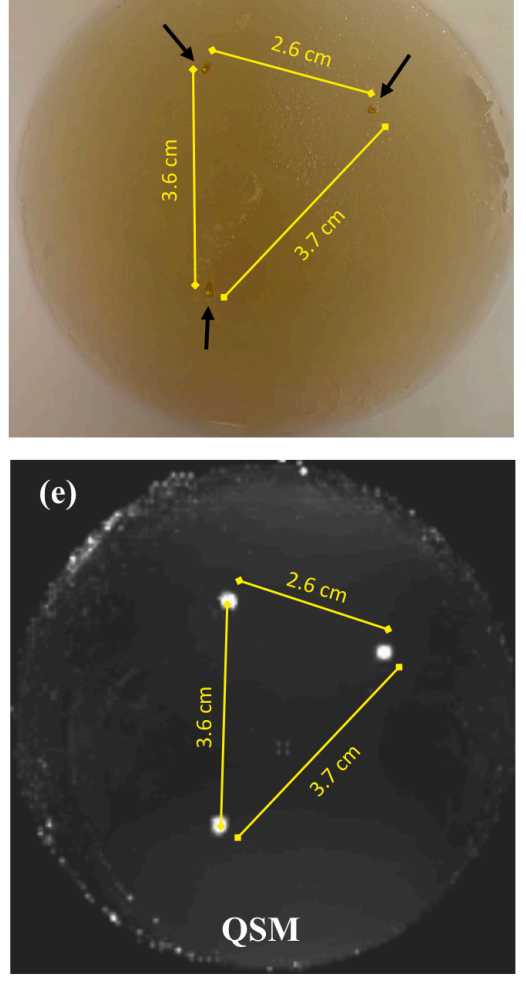

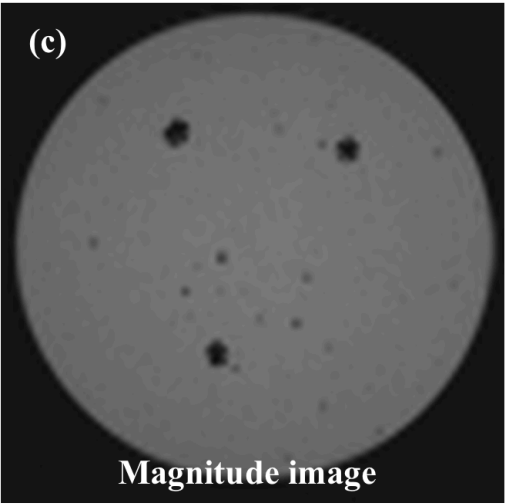

(f)

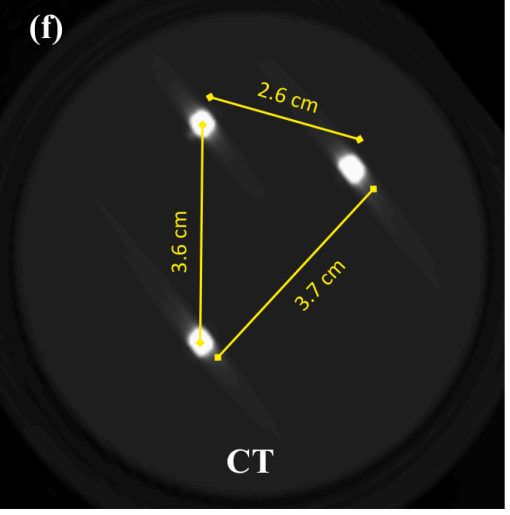

Figure 6.3; (a) structure of the studied gold marker ("CyberMark ${ }^{\mathrm{TM}}$ Fiducial Markers," n.d.); (b) constructed agar-based phantom containing 3 gold makers; (c) MR magnitude image at shortest TE; (d) MR phase image at shortest TE; (e) QSM; (f) CT image 
The relative center-to-center distance between the markers on QSM and CT were $37 \mathrm{~mm}, 36$ $\mathrm{mm}$ and $26 \mathrm{~mm}$ which were identical to the actual measured distance on the phantom.

Figure 6.4a shows the structure of the carbon-coated zirconium (BiomarC) marker. The core material of the marker is pure zirconium with magnetic susceptibility of $109 \mathrm{ppm}$ and the carbon coating has similar susceptibility to the soft tissue. The prostate phantom (Figure 6.4b) was implanted with three markers. After unloading the markers, as shown by the arrow in Figure 6.4c an air bubble as well as the needle tracks were left in the phantom.

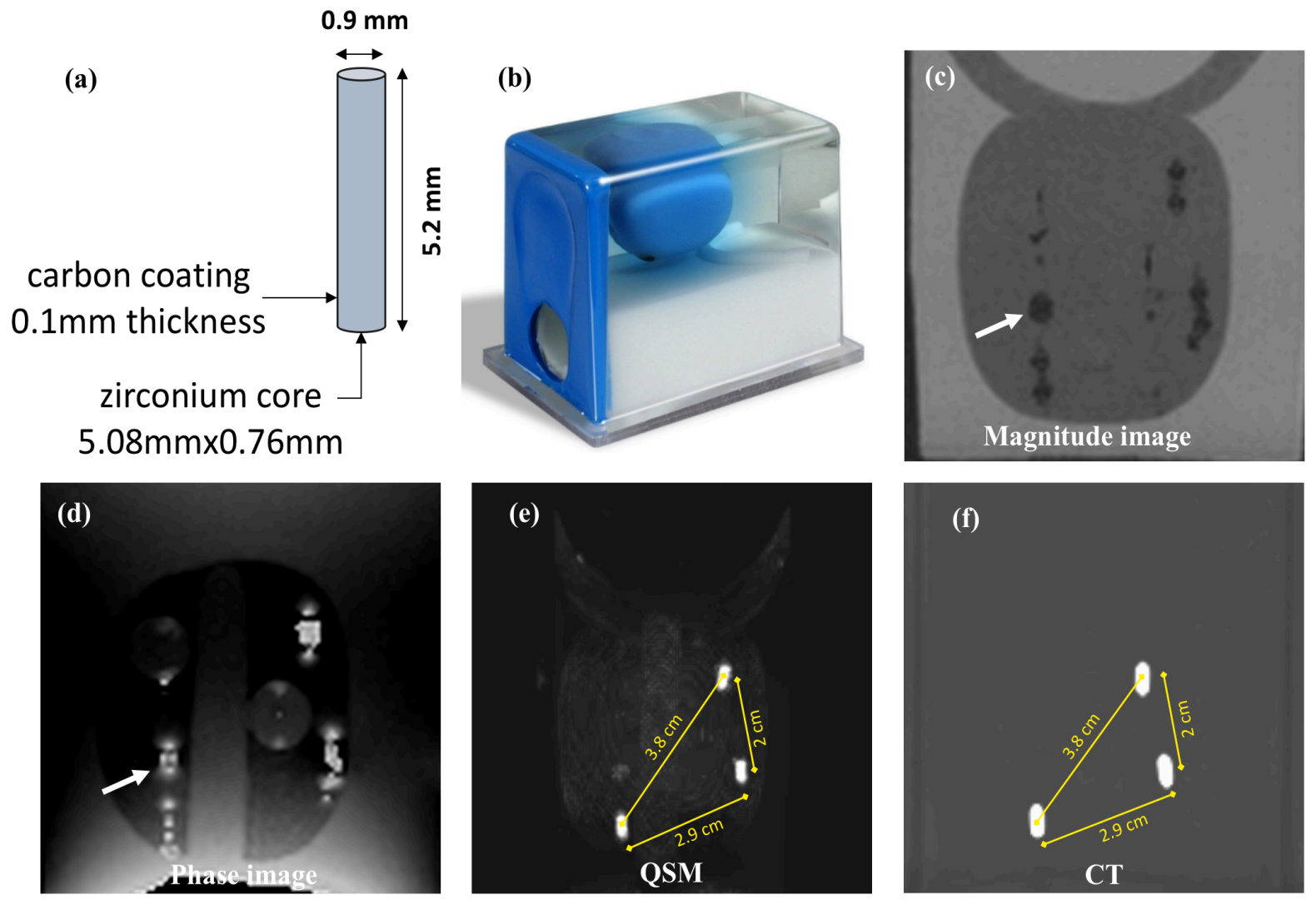

Figure 6.4; (a) structure of the carbon-coated zirconium marker (BiomarC); (b) prostate phantom containing 3 carbon makers; (c) MR magnitude image at shortest TE; (d) MR phase image at shortest TE; (e) reconstructed susceptibility map (QSM); (f) CT image. The arrows in (c) and (d) indicate an air bubble that was left in the phantom along the needle track during implantation however, it did not affect the QSM process. 
In the reconstructed QSM (Figure 6.4e) only implanted markers were depicted with positive contrast and none of the needle tracks were visualized. The average length of the carbon markers on CT and QSM were $5 \pm 0.2 \mathrm{~mm}$ and $4.7 \pm 0.3 \mathrm{~mm}$ respectively. As demonstrated in Figure 6.4e-f, on both CT and QSM the center-to-center distance between the carbon markers were $38 \mathrm{~mm}, 29$ $\mathrm{mm}$ and $20 \mathrm{~mm}$. As shown in Table 2 the average estimated magnetic susceptibility value for the "pure" gold marker was $-36 \pm 4$ ppm which agrees with the expected value of -34 ppm for gold; however, the average estimated susceptibility value for BiomarC markers was $86 \pm 4$ ppm which shows about $20 \%$ compared with pure zirconium which might be due to the carbon coating.

\subsubsection{HDR interstitial brachytherapy needles, markers and obturators}

Figure 6.5a-c shows the hollow plastic needle (sharp tip), marker and obturator and the agar/meat phantoms. The Maximum Intensity Projection (MIP) reconstruction of the QSM and CT images of the phantoms with inserted needles, markers and obturators are shown in Figure 6.5d-f. The ProGuide obturator is made of a Titanium alloy with high susceptibility contrast with the diamagnetic background thus in both agar and meat phantoms the needle with ProGuide obturator had excellent positive contrast on QSM. The CT-marker is a flexible aluminum based wire in a polyimide coating but has strongly paramagnetic nitinol (nickel and titanium) component in it; The CT-marker overall has sufficient susceptibility contrast with background and appeared with positive contrast on QSM. As shown in Figure 6.5e, the two air-filled hollow plastic needles were poorly visualized in the QSM; this was expected as the only paramagnetic component of the air is molecular oxygen which is very weakly paramagnetic (Table 1 ).

The pure titanium rod (without plastic needle) has very good positive contrast on the reconstructed QSM (Figure 6.5d). 
As shown in Figure 6.5a, to localize the tip of the interstitial needle during treatment planning and delivery there is a $10 \mathrm{~mm}$ gap (spacer) near the tip of the CT-marker; The length of the spacer on CT and QSM was $8.5 \mathrm{~mm}$ and $7.5 \mathrm{~mm}$ respectively.
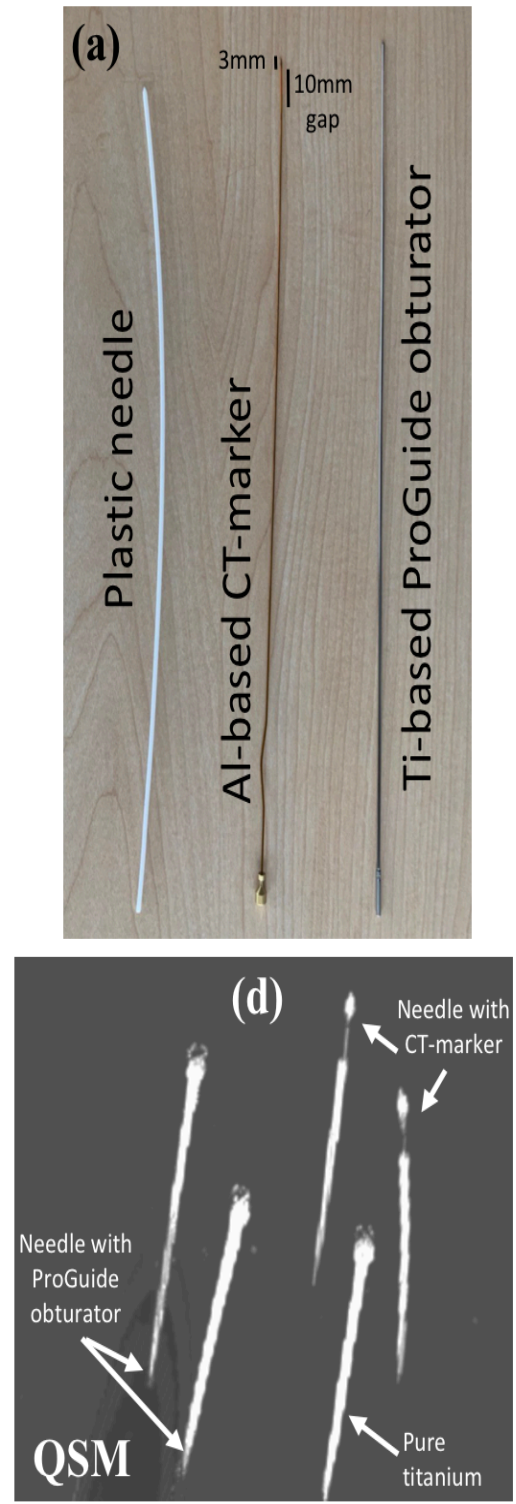

(b)
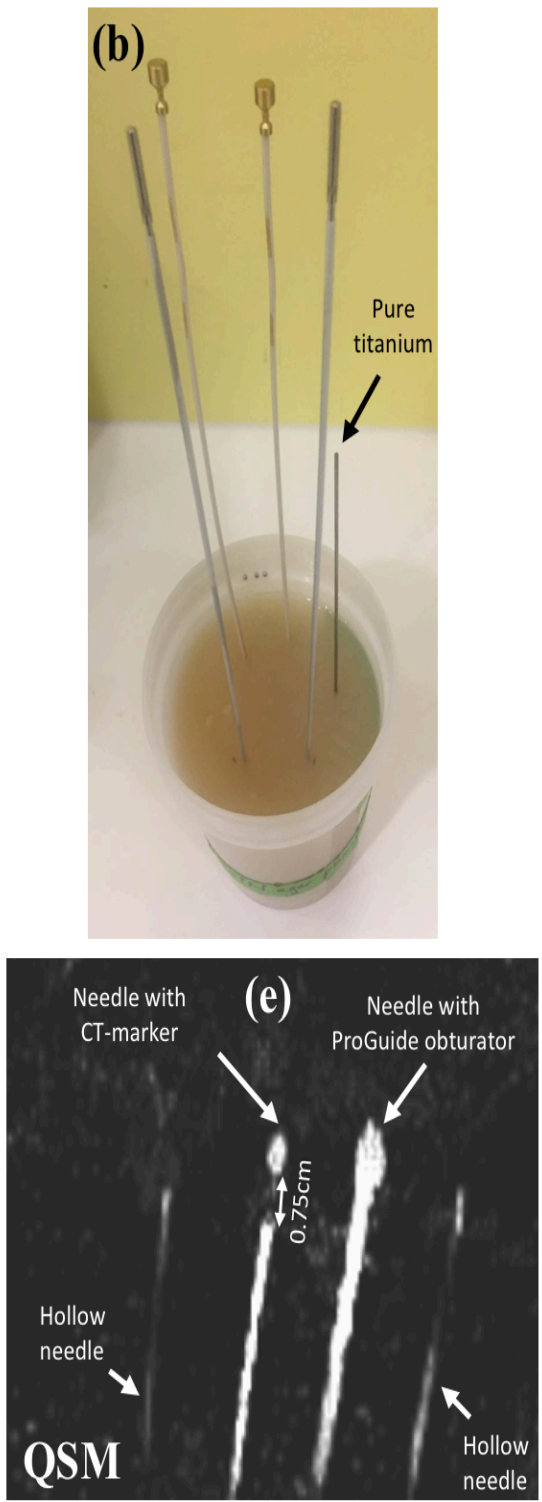
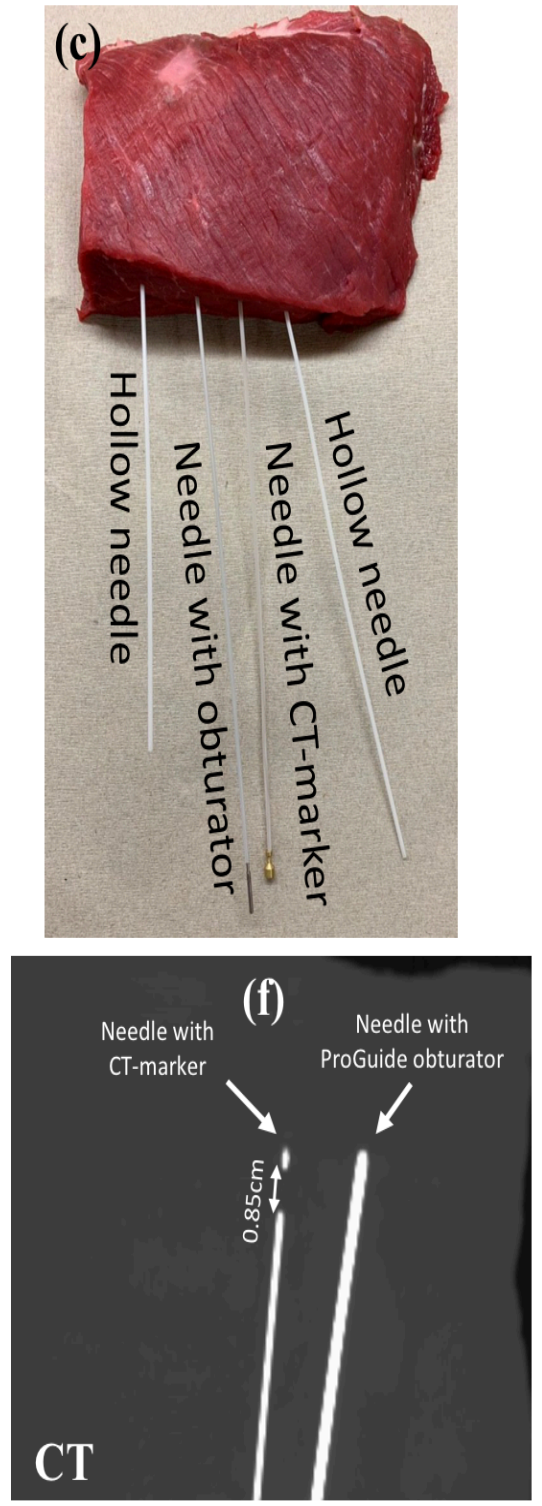

Figure 6.5; (a) Plastic needle, CT-marker and ProGuide obturators that were studied; (b) constructed agar phantom with 5 inserts including 2 plastic needles with CT-marker obturators, 2 plastic needles with Pro-Guide obturators and one pure titanium rod representing a biopsy needle; (c) meat phantom with 2 hollow plastic needles, one needle with CT-marker and one needle with Pro-Guide obturator; (d) MIP reconstruction of the QSM for the agar-based phantom; (e) MIP reconstruction of the QSM of the meat phantom; (f) MIP reconstruction of the CT of the meat phantom. 
The average estimated susceptibility values for CT-marker and ProGuide obturator measured in the meat phantom are presented in Table 6.2.

Table 6.2; The average estimated magnetic susceptibility values for different investigated devices/materials

\begin{tabular}{lllllll} 
& \multicolumn{5}{c}{ Device/object } \\
\cline { 2 - 6 } & $\begin{array}{l}\text { Brachytherapy } \\
\text { seed }\end{array}$ & $\begin{array}{l}\text { Calcification } \\
\text { (bone) }\end{array}$ & $\begin{array}{l}\text { Gold } \\
\text { fiducial } \\
\text { marker }\end{array}$ & $\begin{array}{l}\text { BiomarC } \\
\text { fiducial } \\
\text { marker }\end{array}$ & $\begin{array}{l}\text { Ti-based } \\
\text { ProGuide } \\
\text { obturator }\end{array}$ & $\begin{array}{l}\text { Al-based } \\
\text { CT-marker }\end{array}$ \\
\hline $\begin{array}{l}\text { Average estimated } \\
\text { susceptibility value } \\
\text { (Mean } \pm \text { SD) }(\mathbf{p p m})\end{array}$ & $62 \pm 8$ & $-21 \pm 3$ & $-36 \pm 4$ & $86 \pm 4$ & $174 \pm 16$ & $54.49 \pm 10$ \\
\hline
\end{tabular}

\subsubsection{Performance comparison between the MEDI and MEDI+0}

The comparison between the performance of the post-processing pipeline with MEDI and MEDI+0 in different investigated applications is shown in Figure 6.6. The background segmentation by generating a binary mask which excludes signal voids on the magnitude image (Figure 6.6, second row) reduced susceptibility artifacts around implanted

objects with high susceptibility (seeds, markers and obturators) and resulted in more uniform background. The improved quality of the QSM results by applying MEDI+0 technique compared to the MEDI was more evident in more realistic phantoms including the prostate phantom with implanted carbon markers as well as meat phantom with inserted needles, markers and obturators (Figure 6.6a-b). 
(a) Meat phantom with needles and obturators
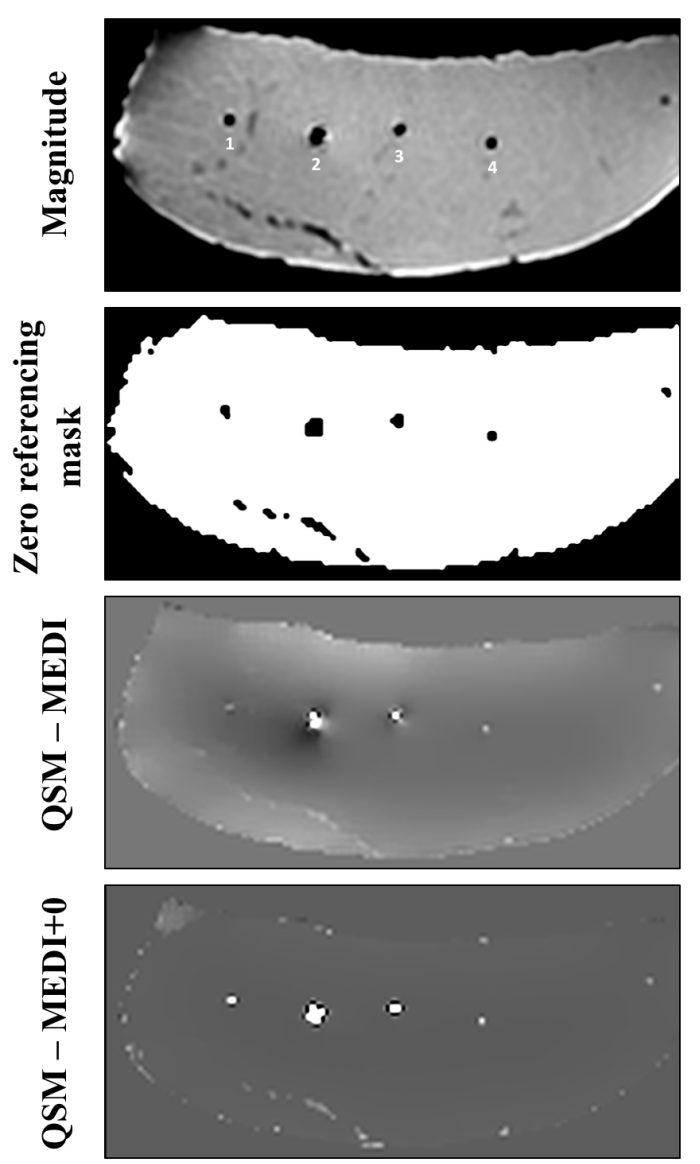

(b) Prostate phantom with carbon fiducial markers
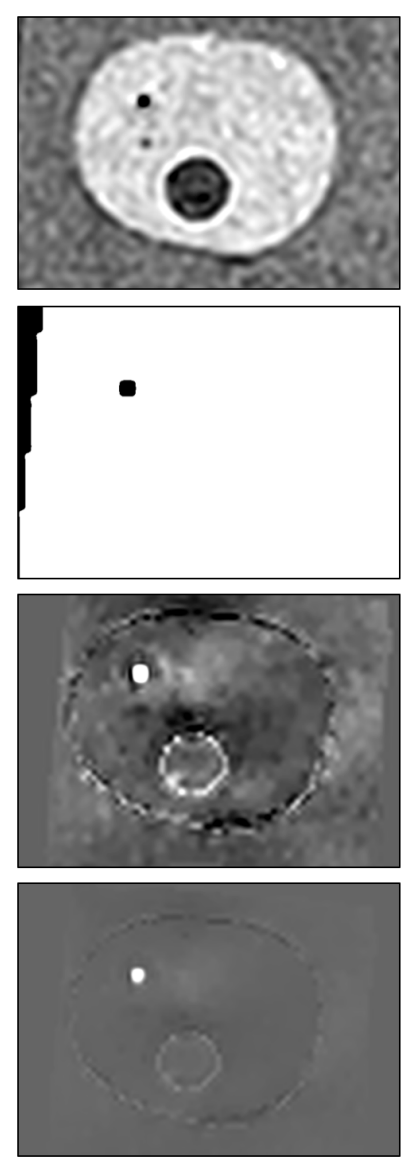

(c) Agar phantom with gold fiducial markers
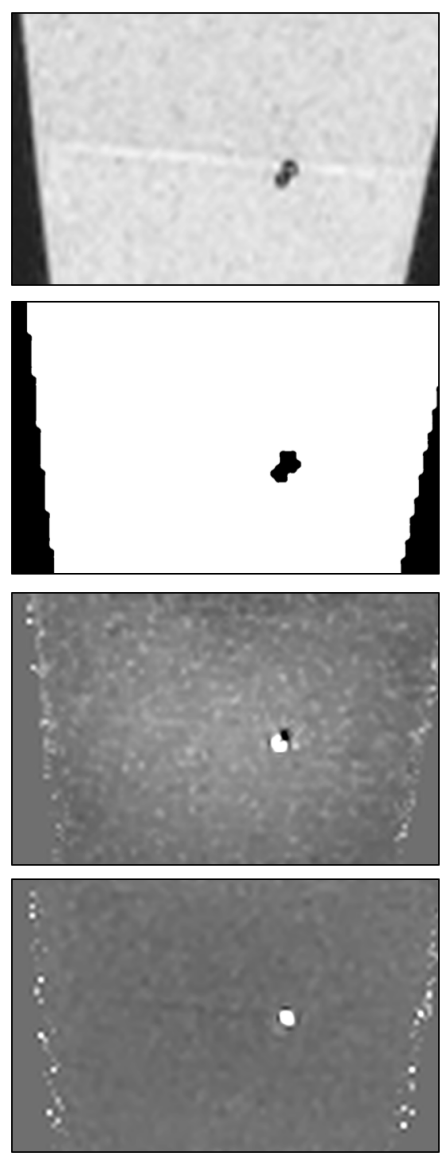

Figure 6.6; The comparison between the QSM results using MEDI and MEDI+0 in (a) meat phantom with two hollow plastic needles (1 and 4) and Pro-Guide obturator (2) and CT-marker (3); (b) prostate phantom with three implanted carbon fiducial markers; (c) agar-based phantom containing three

\subsection{Discussion and Conclusions}

MRI-guided radiation treatment planning and delivery allows for truly adaptive radiation therapy by accurate localization of the tumor and normal surrounding tissue which are critical for optimal treatment outcome for patients. Many of the radiation therapy techniques such as LDR and HDR brachytherapy involve temporary insertion or permanent implantation of MR-invisible 
metallic devices hence CT remains an unavoidable part of such procedures. Efforts have been made to wipe out CT from the current workflows to avoid the unnecessary dose, MR/CT image registration error, and to make efficient resource utilization.

In this work, the performance of the previous method (Nosrati et al., 2018) was improved by applying the MEDI +0 technique, some concerns regarding LDR seeds were addressed and other potential applications for MR-guided radiation therapy were studied. We showed that by applying clinically available MR pulse sequences and only through image post-processing (QSM), conventional paramagnetic MR-invisible radiation therapy devices (such as brachytherapy seeds, CT-marker, Obturator, fiducial markers) are depicted with excellent positive contrast and high spatial accuracy.

Prostatic calcifications are a common finding in men (mostly above 50 years old)(Hong et al., 2012); calcification and seed are not differentiable on MR magnitude images since both appear as dark voids however, they can be easily differentiated from one another using QSM (Dong et al., 2015; Nosrati et al., 2018; Shi et al., 2017). In application of QSM for visualization of implanted prostate seeds one potential concern may be the interference of seed-induced field disturbance with that of nearby calcifications which could results in erroneous frequency map and susceptibility estimation. Our results showed that the proposed algorithm was not affected by the presence of calcifications in the vicinity of the seeds. We also observed that QSM easily differentiates between diamagnetic calcifications and paramagnetic seeds.

The type of implanted seed is another factor that may affect the seed visualization and detection process as the seed-induced field distortion changes with the structure of the seed. Zijlstra et. al. have shown that the performance of seed detection based on field distortion strongly depends on seed type; for instance the false positive seed detection was $42 \%$ for Best 2301 seeds, but $17 \%$ 
for SelectSeed (Zijlstra et al., 2017). Our results for the three types of seeds suggested that if the external dimensions (typically $4.5 \mathrm{~mm}$ by $0.8 \_\mathrm{mm}$ ) and the material of the seed shell (which is usually titanium) are similar the QSM algorithm is not affected by type of seed and leads to similar reconstruction of the seeds. The clinical feasibility of the proposed workflow for post-implant dosimetry of prostate LDR brachytherapy is currently being evaluated on a large cohort of patients and the results have been very promising.

Fiducial markers are commonly used in EBRT for patient setup and tumor tracking. Previous works in MR-based fiducial marker detection are mainly based on template matching using either signal voids on magnitude images or field distortions on phase images; although the aforementioned methods have been effective under certain conditions (orientation, dimensions, shape), clear marker depiction with positive contrast similar to CT was not achieved (Bouwman, Custers, Bakker, Viergever, \& Seevinck, 2019; Ghose et al., 2016; Jonsson, Garpebring, Karlsson, \& Nyholm, 2012). Our results with QSM showed that the relative location of the markers was identical with the CT image indicating the possibility of using QSM in MR-guided EBRT for the disease sites like prostate, liver and lung.

Currently CT-markers are placed in interstitial brachytherapy needles during CT-scans for the reconstruction of these needles required for treatment planning. In addition to CT, MRI is used for contouring the tumor and surrounding organs. Our results showed the possibility of using same commercial CT marker with MRI and QSM avoiding the need for CT imaging. Further validation testing is required on patients, incorporating a higher density of needles, needles that overlap as well as heterogeneous patient anatomy.

In addition to the $\mathrm{CT}$ marker, we evaluated the reconstruction of the commercial obturators used in clinic to guide these needles inside a patient. Obturators are typically not used in static 
images for treatment planning because when they are placed within the hollow catheters their stiffness distorts the surrounding anatomy. It may be possible to make smaller diameter obturators with similar materials that do not perturb the surrounding anatomy for use in MRI. Due to sharp susceptibility gradient between titanium and surrounding soft-tissue, the diameter of the obturator and titanium rod were overestimated by about $20 \%$ and the effect was more pronounced at the tip of the obturator and rod (Figure 5d-e) however, this should not lead to localization error since centroids of the reconstructed hyperintensities are used for localization. The Al-based CT-marker with the gap near the tip, did not get affected with this problem due to smaller susceptibility difference; the centroid of the marker tip which is used to find the first dwell position in the needle, remained unchanged. Similar design (with material gap at the tip) with the material of obturator can possibly be tested in future with our method for their possible use as MR markers. The performance of the proposed method on the pure titanium rod with similar diameter to a typical biopsy needle was similar to the ProGuide obturator. Shi et. al. have also studied the feasibility of susceptibility-based positive contrast visualization of the biopsy needle and LDR seeds however, they used a modified spin-echo (SE) MR sequence with a manually adjusted shifted echo times; the seed visualization is relatively poor and not comparable with CT(Shi et al., 2017). The use of 2D SE sequence with very low readout bandwidth leads to higher susceptibility-induced distortions which are of main concerns in MR-guided procedures.

Our results indicate that MEDI +0 significantly improves the homogeneity of the background and reduces artifacts and hypointense shadows at the boundaries of sharp susceptibility mismatch. Fiducial markers in external beam radiotherapy are used for setup verification using cone beam CT. 
One of the main limitations of this study was the possibility of soft tissue distortions, physiological noise, and the geometrical fidelity in patients which needs to be tested in vivo. Also, the optimal regularization parameters $\left(\lambda_{1}\right.$ and $\left.\lambda_{2}\right)$ were identified experimentally based on the quality of the QSM reconstruction; although for all investigated applications the final optimized value was 100 for both $\lambda_{1}$ and $\lambda_{2}$, in patients they might need to be optimized further depending on the application. The last but not least limitation is the extra MR scan time in addition to the standard MR protocols for anatomical delineation. In both HDR and LDR brachytherapy patients the MRGRE image acquisition time for QSM will add up to 10 minutes extra scan time to the standard MRI for target delineation; the complete image post-processing algorithm for seed/marker visualization and localization (which is fully automated) takes about 8-10 minutes. So overall in brachytherapy patients the MR-only seed detection will take up to 20 minutes in total. This additional time is reasonable considering that in standard MR-CT fusion-based post-planning workflows in addition to the extra CT scan, the MR-CT fusion takes much longer than that 20 minutes and registration could be a source of error as well. Although longer MR scan time makes the process more susceptible to motion artifacts, this may get around the uncertainties associated with the MR-CT registration. The feasibility and reliability of the QSM for the proposed clinical applications will be investigated in patients in the near future.

\subsection{Acknowledgement}

R.N. was funded by the NSERC CGS-D3 doctoral scholarship and the study was funded by Prostate Cancer Canada funding. 
We would like to thank IsoAid and IsoSeed (Eckert and Ziegler) companies for providing brachytherapy seeds. We are also thankful to the CIVCO Radiotherapy and Carbon Medical Technologies for providing fiducial markers. We are grateful to Dr. Mark Logsdon at Sutter Medical Centre for sharing some original ideas and Dr. Alex Karotki at Sunnybrook Health Sciences Centre for his help with the CT scans. 


\section{CHAPTER 7}

\section{CONCLUSION and FUTURE DIRECTIONS}

\subsection{Conclusion}

Radiation therapy techniques have a significant role in cancer treatment. Magnetic Resonance-Guided Radiation Therapy (MR-GRT) offers great potential to improve radiation therapy outcomes by providing more accurate and patient-tailored treatment. Despite poor soft tissue contrast, CT scan is currently an inevitable part of all radiation treatment planning and/or delivery processes that require temporary or permanent placement of paramagnetic objects within the target volume either for patient positioning or for accurate treatment delivery. Low-Dose-Rate (LDR) brachytherapy is an established treatment for low-risk prostate cancer patients, which involves permanent implantation of several titanium encapsulated radiation sources in the prostate. The current MRI-CT fusion-based workflow for post-implant dosimetry of LDR prostate brachytherapy takes advantage of the superior soft tissue contrast of MRI but still relies on CT for seed visualization and detection. Lack of positive contrast from implanted brachytherapy seeds has been the major challenge towards an MRI-only post-implant dosimetry workflow.

This work aimed at developing an MRI-only workflow for post-implant dosimetry of prostate LDR brachytherapy. The goal was to propose a clinically feasible pipeline that does not require any hardware or software modifications and solely relies on MR post-processing algorithms. 
In the first step, the feasibility of several MRI contrast mechanisms including T1-mapping, T2/T2*-mapping, proton density mapping, ultra-short echo time (UTE) sequence and Quantitative Susceptibility Mapping (QSM) for brachytherapy seed detection (in the presence of calcifications) were studied. Among all the assessed contrast mechanisms, QSM showed the best results to visualize seeds with distinctive positive contrast. A QSM algorithm was developed for brachytherapy seed depiction in simple agar-based phantoms as well as realistic prostate phantoms with clinically relevant seed configurations. To compare and validate the MR-based seed positions and dosimetry with those of the standard CT-based approach, a machine learning algorithm was introduced for seed detection and localization. Following validation of the proposed MRI-based pipeline on phantoms, the efficiency of the algorithm was investigated on LDR prostate patients. The clinical translation of the proposed workflow required extensive optimizations in image acquisition parameters and post-processing pipeline. In addition to brachytherapy seeds, other applications of the QSM for positive contrast depiction of some other radiation therapy devices including EBRT fiducial markers and interstitial brachytherapy needles, catheters and CT-marker stylets in phantoms were investigated.

In this thesis, a clinically reliable MRI-only workflow for post-implant positive contrast seed visualization and localization of brachytherapy seeds is presented. Seed visualization, localization and dosimetry were compared between the proposed MRI-only workflow and the standard CT-based and CT-MRI fusion-based approaches and no significant difference was observed between the two methods. A submillimeter average difference was observed between MR and CT-derived seed positions, which may be due to prostate deformations between the MR and CT scans which may have caused small discrepancies between relative seed positions between the two methods. The main benefits of the developed workflow in comparison with the previous 
works are relying only on image post-processing and being applicable to conventional brachytherapy seeds.

In addition, the feasibility of the proposed method on some other MR-invisible radiation therapy devices was studied and showed excellent performance in phantom studies. In general, an MRI-only guided radiation therapy workflow eliminates the uncertainties associated with the MRCT fusion and improves the quality of the treatment.

In conclusion, the MRI-only post-implant dosimetry using QSM has shown to be feasible and accurate and offers a unique opportunity to replace the conventional CT-only or MR-CT fusionbased workflows.

\subsection{Future directions}

This thesis was focused on developing an MRI-only workflow for post-implant dosimetry of prostate LDR brachytherapy using QSM. Although the proposed workflow was successfully validated on a relatively large cohort of patients, a few patients were excluded from the analysis due to severe motion artifacts. In all patients, the anterior-posterior direction was chosen as frequency encoding direction, which is known to be less susceptible to motion artifacts. However, motion artifacts in the readout direction were observed in 2 (out of 25) study patients, which were excluded from the analysis. The quality of the QSM and resulting seed localization and dosimetry are susceptible to motion artifacts, specifically breathing and internal movement of the structures near the prostate such as the rectum, small bowel, and bladder. A future extension of this study may be a re-evaluation of this method after administration of bowel movement suppressors such as glutamine before MR scan and investigate its potential benefits. In addition, the current image acquisition time for QSM is 10 minutes, which may further make the process susceptible to motion 
artifacts. Reducing the scan time by optimizing pulse sequence parameters potentially improves the efficiency of the workflow; however, there is a tradeoff between the scan time and the image SNR. Therefore, a clinical study to determine optimal sequence parameters that allow for minimal scan time with sufficient SNR for QSM analysis.

Some potential applications of the proposed workflow in MR-GRT were investigated in this thesis. However, the clinical feasibility of them was not assessed. Considering the recently introduced MR-Linac hybrid system the clinical evaluation of the proposed method for MR-based identification of the EBRT fiducial markers, HDR catheters and obturators could be a very interesting future study given the vast applications of them in routine radiation therapy practice.

The current clinical applications of QSM are mainly limited to the brain for certain diseases where the susceptibility differences are below $50 \mathrm{ppm}$, which results in minor image distortions. In addition to the magnitude of the susceptibility gradient, the MR distortions are directly related to the volume of the high susceptibility region. The present study suggests that the proposed QSM pipeline efficiently visualize small metallic objects of large susceptibility gradients $(>150 \mathrm{ppm})$ with high spatial accuracy, however, the effectiveness of this process will degrade with increasing the volume of the object with high magnetic susceptibility. For instance, the new MR-compatible intracavitary brachytherapy probes (usually made of titanium) used in gynecological cancers generate large distortions on MR image. Evaluation and optimization of the proposed QSM-based method on larger metallic devices such as HDR probes, metallic prosthesis, and surgical screws are recommended.

In terms of improvement of the QSM algorithm for highly paramagnetic materials such as titanium implants, there are potential strategies to improve different steps of the QSM process. The 
phase unwrapping around seeds is very challenging due to the small size of the seeds. The most widely used phase unwrapping technique is the Laplacian-based phase unwrapping with the assumption of phase continuity in both image and Fourier domain, however, this assumption can lead to significant errors in case of a very small but highly susceptible object such as brachytherapy seeds. In this thesis, different phase unwrapping algorithms were studied, and a quality-guided phase unwrapping was found to be the most efficient one. However, it still resulted in errors at specific seed orientations with respect to the main magnetic field. A novel phase unwrapping technique with efficient unwrapping regardless of the seed orientation will improve the reliability of QSM. In addition, currently, different steps of the QSM process are performed by solving three different optimization problems. Feasibility of an alternating minimization scheme for joint estimation of the local field and susceptibility map is worth to investigate; this joint estimation potentially reduces the computational time, as well as the solution, may improve the quality of the QSM calculation. 


\section{REFERENCES}

Adjeiwaah, M., Bylund, M., Lundman, J. A., Karlsson, C. T., Jonsson, J. H., \& Nyholm, T. (2018). Quantifying the Effect of 3T Magnetic Resonance Imaging Residual System Distortions and Patient-Induced Susceptibility Distortions on Radiation Therapy Treatment Planning for Prostate Cancer. International Journal of Radiation Oncology*Biology*Physics, 100(2), 317-324. doi:10.1016/J.IJROBP.2017.10.021

Afsharpour, H., D’Amours, M., Coté, B., Carrier, J.-F., Verhaegen, F., \& Beaulieu, L. (2008). A Monte Carlo study on the effect of seed design on the interseed attenuation in permanent prostate implants. Medical Physics, 35(8), 3671-3681. doi:10.1118/1.2955754

Ash, D., Flynn, A., Battermann, J., de Reijke, T., Lavagnini, P., Blank, L., ... EORTC Radiotherapy Group. (2000). ESTRO/EAU/EORTC recommendations on permanent seed implantation for localized prostate cancer. Radiotherapy and Oncology : Journal of the European Society for Therapeutic Radiology and Oncology, 57(3), 315-21.

Beaulieu, L., Tedgren, A., \& Carrier, J. (2012). Report of the Task Group 186 on model-based dose calculation methods in brachytherapy beyond the TG-43 formalism: Current status and recommendations for clinical implementation. Medical Physics, 39(10), 6208-6236.

Berkhin, P. (2006). A Survey of Clustering Data Mining Techniques. In Grouping Multidimensional Data (pp. 25-71). Berlin/Heidelberg: Springer-Verlag. doi: $10.1007 / 3.540-28349-8 \_2$

Besl, P. J., \& McKay, N. D. (1992). A method for registration of 3-D shapes. IEEE Transactions 
on Pattern Analysis and Machine Intelligence, 14(2), 239-256. doi:10.1109/34.121791

Bilgic, B., Chatnuntawech, I., Fan, A. P., Setsompop, K., Cauley, S. F., Wald, L. L., \& Adalsteinsson, E. (2014). Fast image reconstruction with L2-regularization. Journal of Magnetic Resonance Imaging, 40(1), 181-191. doi:10.1002/jmri.24365

Bioucas-Dias, J. M., \& Valadão, G. (2007). Phase Unwrapping via Graph Cuts. IEEE TRANSACTIONS ON IMAGE PROCESSING, 16(3). doi:10.1109/TIP.2006.888351

Bloch, B. N., Lenkinski, R. E., Helbich, T. H., Ngo, L., Oismueller, R., Jaromi, S., ... Rofsky, N. M. (2007). Prostate postbrachytherapy seed distribution: comparison of high-resolution, contrast-enhanced, T1- and T2-weighted endorectal magnetic resonance imaging versus computed tomography: initial experience. International Journal of Radiation Oncology, Biology, Physics, 69(1), 70-8. doi:10.1016/j.ijrobp.2007.02.039

Boonsirikamchai, P., Choi, S., Frank, S. J., Ma, J., Elsayes, K. M., Kaur, H., \& Choi, H. (2013). MR imaging of prostate cancer in radiation oncology: what radiologists need to know. Radiographics : A Review Publication of the Radiological Society of North America, Inc, 33(3), 741-61. doi:10.1148/rg.333125041

Bouwman, J. G., Custers, B. A., Bakker, C. J. G., Viergever, M. A., \& Seevinck, P. R. (2019). isoPhasor: a generic and precise marker visualization, localization, and quantification method based on phase saddles in 3D MR imaging. Magnetic Resonance in Medicine, 81(3), 2038-2051. doi:10.1002/mrm.27493

Bowes, D., Crook, J. M., Araujo, C., \& Batchelar, D. (2013). Ultrasound-CT fusion compared 
with MR-CT fusion for postimplant dosimetry in permanent prostate brachytherapy. Brachytherapy, 12(1), 38-43. doi:10.1016/J.BRACHY.2012.03.007

Buch, K., Morancy, T., Kaplan, I., Qureshi, M. M., Hirsch, A. E., Rofksy, N. M., ... Bloch, N. (2014). Improved dosimetry in prostate brachytherapy using high resolution contrast enhanced magnetic resonance imaging: a feasibility study. Journal of Contemporary Brachytherapy, 4(4), 337-343. doi:10.5114/jcb.2014.46555

Carrier, J.-F., Beaulieu, L., Therriault-Proulx, F., \& Roy, R. (2006). Impact of interseed attenuation and tissue composition for permanent prostate implants. Medical Physics, 33(3), 595-604. doi:10.1118/1.2168295

Carrier, J.-F., D’Amours, M., Verhaegen, F., Reniers, B., Martin, A.-G., Vigneault, E., \& Beaulieu, L. (2007). Postimplant dosimetry using a Monte Carlo dose calculation engine: a new clinical standard. International Journal of Radiation Oncology, Biology, Physics, 68(4), 1190-8. doi:10.1016/j.jijrobp.2007.02.036

Chen, P. Y. (2009). 10-year results of interstitial needle-catheter brachytherapy for accelerated partial breast irradiation: Excellent control rates with minimal toxicities. Brachytherapy, $8(2)$.

Chen, W., Gauthier, S. A., Gupta, A., Comunale, J., Liu, T., Wang, S., ... Wang, Y. (2014). Quantitative susceptibility mapping of multiple sclerosis lesions at various ages. Radiology, 271(1), 183-92. doi:10.1148/radiol.13130353

Chin, J., Rumble, R. B., Kollmeier, M., Heath, E., Efstathiou, J., Dorff, T., ... Loblaw, D. A. 
(2017). Brachytherapy for Patients With Prostate Cancer: American Society of Clinical Oncology/Cancer Care Ontario Joint Guideline Update. Journal of Clinical Oncology, 35(15), 1737-1743. doi:10.1200/JCO.2016.72.0466

Chollet, F. (2015). Keras.

Collins Fekete, C.-A., Plamondon, M., Martin, A.-G., Vigneault, É., Verhaegen, F., \& Beaulieu, L. (2014). Quantifying the effect of seed orientation in postplanning dosimetry of low-doserate prostate brachytherapy. Medical Physics, 41(10), 101704. doi:10.1118/1.4895012

Cronin, K. A., Lake, A. J., Scott, S., Sherman, R. L., Noone, A.-M., Howlader, N., ... Jemal, A. (2018). Annual Report to the Nation on the Status of Cancer, part I: National cancer statistics. Cancer, 124(13), 2785-2800. doi:10.1002/cncr.31551

CyberMark $^{\mathrm{TM}}$ Fiducial Markers. (n.d.). Retrieved February 12, 2019, from https://civcort.com/ro/fiducial-markers/cybermark-gold-markers/cybermark-markerFMC11.htm

Dahnke, H., Liu, W., Herzka, D., Frank, J. A., \& Schaeffter, T. (2008). Susceptibility gradient mapping (SGM): a new postprocessing method for positive contrast generation applied to superparamagnetic iron oxide particle (SPIO)-labeled cells. Magnetic Resonance in Medicine, 60(3), 595-603. doi:10.1002/mrm.21478

Dao-Qiang Zhang, Song-Can Chen, Zhi-Song Pan, \& Ke-Ren Tan. (n.d.). Kernel-based fuzzy clustering incorporating spatial constraints for image segmentation. In Proceedings of the 2003 International Conference on Machine Learning and Cybernetics (IEEE Cat. 
No.03EX693) (pp. 2189-2192). IEEE. doi:10.1109/ICMLC.2003.1259869

Davis, B. J., Horwitz, E. M., Lee, W. R., Crook, J. M., Stock, R. G., Merrick, G. S., ... American Brachytherapy Society. (2012). American Brachytherapy Society consensus guidelines for transrectal ultrasound-guided permanent prostate brachytherapy. Brachytherapy, 11(1), 619. doi:10.1016/j.brachy.2011.07.005

De Brabandere, M., Al-Qaisieh, B., De Wever, L., Haustermans, K., Kirisits, C., Moerland, M. A., ... Siebert, F.-A. (2013). CT- and MRI-based seed localization in postimplant evaluation after prostate brachytherapy. Brachytherapy, 12(6), 580-588. doi:10.1016/j.brachy.2013.06.003

De Brabandere, M., Hoskin, P., Haustermans, K., Van den Heuvel, F., \& Siebert, F.-A. (2012). Prostate post-implant dosimetry: Interobserver variability in seed localisation, contouring and fusion. Radiotherapy and Oncology, 104(2), 192-198. doi:10.1016/J.RADONC.2012.06.014

De Brabandere, M., Hoskin, P., Haustermans, K., Van Den Heuvel, F., \& Siebert, F. A. (2012). Prostate post-implant dosimetry: Interobserver variability in seed localisation, contouring and fusion. Radiotherapy and Oncology, 104(2), 192-198. doi:10.1016/j.radonc.2012.06.014

de Leeuw, H., Seevinck, P. R., \& Bakker, C. J. G. (2013). Center-out radial sampling with offresonant reconstruction for efficient and accurate localization of punctate and elongated paramagnetic structures. Magnetic Resonance in Medicine, 69(6), 1611-1622. doi:10.1002/mrm.24416 
de Leeuw, Hendrik, Moerland, M. A., van Vulpen, M., Seevinck, P. R., \& Bakker, C. J. G. (2013a). A dual-plane co-RASOR technique for accurate and rapid tracking and position verification of an Ir-192 source for single fraction HDR brachytherapy. Physics in Medicine and Biology, 58(21), 7829-39. doi:10.1088/0031-9155/58/21/7829

de Leeuw, Hendrik, Moerland, M. A., van Vulpen, M., Seevinck, P. R., \& Bakker, C. J. G. (2013b). A dual-plane co-RASOR technique for accurate and rapid tracking and position verification of an Ir-192 source for single fraction HDR brachytherapy. Physics in Medicine and Biology, 58(21), 7829-7839. doi:10.1088/0031-9155/58/21/7829

de Rochefort, L. (2010). Quantitative susceptibility map reconstruction from MR phase data using bayesian regularization: Validation and application to brain imaging. Magnetic Resonance in Medicine, 63(1).

de Rochefort, L., Brown, R., Prince, M. R., \& Wang, Y. (2008). Quantitative MR susceptibility mapping using piece-wise constant regularized inversion of the magnetic field. Magnetic Resonance in Medicine, 60(4), 1003-1009. doi:10.1002/mrm.21710

Dehghan, E., Le, Y., Lee, J., Song, D. Y., Fichtinger, G., \& Prince, J. L. (2016). CT AND MRI FUSION FOR POSTIMPLANT PROSTATE BRACHYTHERAPY EVALUATION. Proceedings. IEEE International Symposium on Biomedical Imaging, 2016, 625-628. doi:10.1109/ISBI.2016.7493345

Del Grande, F., Santini, F., Herzka, D. A., Aro, M. R., Dean, C. W., Gold, G. E., \& Carrino, J. A. (2014). Fat-Suppression Techniques for 3-T MR Imaging of the Musculoskeletal System. RadioGraphics, 34(1), 217-233. doi:10.1148/rg.341135130 
Dong, Y., Chang, Z., Xie, G., Whitehead, G., \& Ji, J. X. (2015). Susceptibility-based positive contrast MRI of brachytherapy seeds. Magnetic Resonance in Medicine, 74(3), 716-26. doi:10.1002/mrm.25453

Dubois, D. F., Prestidge, B. R., Hotchkiss, L. A., Prete, J. J., \& Bice, W. S. (1998). Intraobserver and interobserver variability of MR imaging- and CT-derived prostate volumes after transperineal interstitial permanent prostate brachytherapy. Radiology, 207(3), 785-789. doi:10.1148/radiology.207.3.9609905

Edge S.B., Byrd D.R., Compton C.C., Fritz A.G., Greene F.L., T. A. (2010). AJCC Cancer Staging Handbook: from the AJCC Cancer Staging Manual (7th edition). American Joint Commitee on Cancer, 2011. doi:10.1007/s00259-010-1693-9

Erickson, B., Albano, K., \& Gillin, M. (1996). CT-guided interstitial implantation of gynecologic malignancies. International Journal of Radiation Oncology*Biology*Physics, 36(3), 699709. doi:10.1016/S0360-3016(96)00373.2

Ester, M., Kriegel, H.-P., Sander, J., \& Xu, X. (1996). A density-based algorithm for discovering clusters a density-based algorithm for discovering clusters in large spatial databases with noise. Proceedings of the Second International Conference on Knowledge Discovery and Data Mining. AAAI Press.

Fessler, J.A., \& Rogers, W. L. (1996). Spatial resolution properties of penalized-likelihood image reconstruction: space-invariant tomographs. IEEE Transactions on Image Processing, 5(9), 1346-1358. doi:10.1109/83.535846 
Fessler, Jeffrey A. (n.d.). Michigan Image Reconstruction Toolbox. Retrieved August 10, 2018, from https://web.eecs.umich.edu/ fessler/code/

Fessler, Jeffrey A, \& Xed, T. E. (1997). Conjugate-Gradient Preconditioning Methods: Numerical Results.

Fleming, J. A., \& Dewar, J. (1898). On the Magnetic Susceptibility of Liquid Oxygen. In Proceedings of the Royal Society of London (Vol. 63, pp. 311-329). Royal Society. doi:10.2307/115993

Frank, S. J., Stafford, R. J., Bankson, J. a, Li, C., Swanson, D. a, Kudchadker, R. J., \& Martirosyan, K. S. (2008). A novel MRI marker for prostate brachytherapy. International Journal of Radiation Oncology, Biology, Physics, 71(1), 5-8. doi:10.1016/j.ijrobp.2008.01.028

GARZOTTO, M., \& FAIR, W. R. (2000). Historical Perspective on Prostate Brachytherapy. Journal of Endourology, 14(4), 315-318. doi:10.1089/end.2000.14.315

Ghose, S., Mitra, J., Rivest-Hénault, D., Fazlollahi, A., Stanwell, P., Pichler, P., ... Dowling, J. A. (2016). MRI-alone radiation therapy planning for prostate cancer: Automatic fiducial marker detection. Medical Physics, 43(5), 2218-2228. doi:10.1118/1.4944871

Greenlee, R. T., Hill-Harmon, M. B., Murray, T., \& Thun, M. (2001). Cancer statistics, 2001. CA: A Cancer Journal for Clinicians, 51(2), 15-36.

Gregory, K. J., Pattison, J. E., \& Bibbo, G. (2015). Measurement uncertainty analysis of lowdose-rate prostate seed brachytherapy: post-implant dosimetry. Australasian Physical \& 
Engineering Sciences in Medicine, 38(1), 71-81. doi:10.1007/s13246-014-0325-2

Haack, S., Nielsen, S. K., Lindegaard, J. C., Gelineck, J., \& Tanderup, K. (2009). Applicator reconstruction in MRI 3D image-based dose planning of brachytherapy for cervical cancer. Radiotherapy and Oncology, 91(2), 187-193. doi:10.1016/J.RADONC.2008.09.002

Haacke, E. M., Xu, Y., Cheng, Y.-C. N., \& Reichenbach, J. R. (2004). Susceptibility weighted imaging (SWI). Magnetic Resonance in Medicine, 52(3), 612-8. doi:10.1002/mrm.20198

Hargreaves, B. A., Worters, P. W., Pauly, K. B., Pauly, J. M., Koch, K. M., \& Gold, G. E. (2011). Metal-induced artifacts in MRI. American Journal of Roentgenology. doi:10.2214/AJR.11.7364

Hartigan, J. A., \& Wong, M. A. (1979). Algorithm AS 136: A K-Means Clustering Algorithm. Journal of the Royal Statistical Society, 28(1), 100-108. doi:10.2307/2346830

Hattori, K., Ikemoto, Y., Takao, W., Ohno, S., Harimoto, T., Kanazawa, S., ... Kato, H. (2013). Development of MRI phantom equivalent to human tissues for 3.0-T MRI. Medical Physics, 40(3), 032303. doi:10.1118/1.4790023

Henry, E. R., \& Hofrichter, J. (1992). Singular value decomposition: Application to analysis of experimental data. Methods in Enzymology, 210, 129-192. doi:10.1016/00766879(92)10010-B

Hong, C. G., Yoon, B. Il, Choe, H.-S., Ha, U.-S., Sohn, D. W., \& Cho, Y.-H. (2012). The Prevalence and Characteristic Differences in Prostatic Calcification between Health Promotion Center and Urology Department Outpatients. Korean Journal of Urology, 53(5), 
330-4. doi:10.4111/kju.2012.53.5.330

Hoskin, P. (2013). Interstitial Prostate Brachytherapy. (G. Kovacs \& P. Hoskin, Eds.). Springer. doi:10.1007/978-3-642-36499-0

Jackson, J. D. (1999). Classical electrodynamics. Wiley.

Javad, M., Birgani, T., Seif, F., Chegeni, N., \& Bayatiani, M. R. (2012). Determination of the effective atomic and mass numbers for mixture and compound materials in high energy photon interactions. Journal of Radioanalytical and Nuclear Chemistry An International Journal Dealing with All Aspects and Applications of Nuclear Chemistry, 292, 1367-1370. doi:10.1007/s10967-012-1677-2

Jenkinson, M., Wilson, J. L., \& Jezzard, P. (2004). Perturbation method for magnetic field calculations of nonconductive objects. Magnetic Resonance in Medicine, 52(3), 471-477. doi:10.1002/mrm.20194

Jonsson, J. H., Garpebring, A., Karlsson, M. G., \& Nyholm, T. (2012). Internal Fiducial Markers and Susceptibility Effects in MRI—Simulation and Measurement of Spatial Accuracy. International Journal of Radiation Oncology*Biology*Physics, 82(5), 1612-1618. doi:10.1016/J.IJROBP.2011.01.046

Kan, H., Kasai, H., Arai, N., Kunitomo, H., Hirose, Y., \& Shibamoto, Y. (2016). Background field removal technique using regularization enabled sophisticated harmonic artifact reduction for phase data with varying kernel sizes. Magnetic Resonance Imaging, 34(7), 1026-1033. doi:10.1016/j.mri.2016.04.019 
Kapur, T., Egger, J., Damato, A., Schmidt, E. J., \& Viswanathan, A. N. (2012). 3-T MR-guided brachytherapy for gynecologic malignancies. Magnetic Resonance Imaging, 30(9), 12791290. doi:10.1016/j.mri.2012.06.003

Khoo, V. S., Dearnaley, D. P., Finnigan, D. J., Padhani, A., Tanner, S. F., \& Leach, M. O. (1997). Magnetic resonance imaging (MRI): considerations and applications in radiotherapy treatment planning. Radiotherapy and Oncology, 42(1), 1-15. doi:10.1016/S01678140(96)01866-X

Kirby, R. (2018). Men and early prostate cancer. Trends in Urology \& Men's Health, 9(3), 7-10. doi:10.1002/tre.632

Kishan, A. U., \& Kupelian, P. A. (2015). Late rectal toxicity after low-dose-rate brachytherapy: incidence, predictors, and management of side effects. Brachytherapy, 14(2), 148-59. doi:10.1016/j.brachy.2014.11.005

Kjer, H. M., \& Wilm, J. (2010). Evaluation of surface registration algorithms for PET motion correction.

Kressler, B., de Rochefort, L., Tian Liu, T., Spincemaille, P., Quan Jiang, Q., \& Yi Wang, Y. (2010). Nonlinear Regularization for Per Voxel Estimation of Magnetic Susceptibility Distributions From MRI Field Maps. IEEE Transactions on Medical Imaging, 29(2), 273281. doi:10.1109/TMI.2009.2023787

Kroon, D.-J., \& Slump, C. H. (2009). Coherence Filtering to Enhance the Mandibular Canal in Cone-Beam CT data. In Proceedings of the 4th Annual Symposium of the IEEE-EMBS 
Benelux Chapter (pp. 41-44). IEEE EMBS Benelux Chapter.

Kulkarni, N. M., Hong, T. S., Kambadakone, A., \& Arellano, R. S. (2015). CT-Guided Implantation of Intrahepatic Fiducial Markers for Proton Beam Therapy of Liver Lesions: Assessment of Success Rate and Complications. American Journal of Roentgenology, 204(2), W207-W213. doi:10.2214/AJR.14.12901

Kunogi, H., Hojo, H., Wakumoto, Y., Saito, A. I., Ishikura, S., Yamashiro, Y., ... Sasai, K. (2015). A new two-step accurate CT-MRI fusion technique for post-implant prostate cancer. Journal of Contemporary Brachytherapy, 7(2), 117-21. doi:10.5114/jcb.2015.51290

Kuo, N., Lee, J., Tempany, C., Stuber, M., \& Prince, J. (2010a). MRI-BASED PROSTATE BRACHYTHERAPY SEED LOCALIZATION. Proceedings / IEEE International Symposium on Biomedical Imaging: From Nano to Macro. IEEE International Symposium on Biomedical Imaging, 2010, 1397-1400. doi:10.1109/ISBI.2010.5490259

Kuo, N., Lee, J., Tempany, C., Stuber, M., \& Prince, J. (2010b). MRI-BASED PROSTATE BRACHYTHERAPY SEED LOCALIZATION. Proceedings. IEEE International Symposium on Biomedical Imaging, 2010, 1397-1400. doi:10.1109/ISBI.2010.5490259

Larrañaga, P., Calvo, B., Santana, R., Bielza, C., Galdiano, J., Inza, I., ... Robles, V. (2006). Machine learning in bioinformatics. Briefings in Bioinformatics, 7(1), 86-112. doi:10.1093/bib/bbk007

Lee, R. J., Suh, H. S., Lee, K. J., Lim, S., Kim, Y., Kim, S., \& Choi, J. (2007). A magnetic resonance-based seed localization method for I-125 prostate implants. Journal of Korean 
Medical Science, 22 Suppl(Suppl), S129-33. doi:10.3346/jkms.2007.22.S.S129

Lee, W. R., Roach, M., Michalski, J., Moran, B., \& Beyer, D. (2002). Interobserver variability leads to significant differences in quantifiers of prostate implant adequacy. International Journal of Radiation Oncology, Biology, Physics, 54(2), 457-61.

Lee, Y. K., Bollet, M., Charles-Edwards, G., Flower, M. A., Leach, M. O., McNair, H., ... Webb, S. (2003). Radiotherapy treatment planning of prostate cancer using magnetic resonance imaging alone. Radiotherapy and Oncology, 66(2), 203-216. doi:10.1016/S0167$8140(02) 00440-1$

Levitt, S. H., Purdy, J. A., Perez, C. A., \& Poortmans, P. (2012). Technical basis of radiation therapy : practical clinical applications. Springer.

Li, W., Avram, A. V, Wu, B., Xiao, X., \& Liu, C. (2014). Integrated Laplacian-based phase unwrapping and background phase removal for quantitative susceptibility mapping. NMR in Biomedicine, 27(2), 219-27. doi:10.1002/nbm.3056

Li, W., Wu, B., \& Liu, C. (2011). Quantitative susceptibility mapping of human brain reflects spatial variation in tissue composition. NeuroImage, 55(4), 1645-1656. doi:10.1016/j.neuroimage.2010.11.088

Lim, T. (n.d.). Better Toxicity Outcomes With HDR and LDR Brachytherapy in Comparison With External Beam Radiation Therapy for Prostate Cancer, 93(Supplement).

Lim, T. Y., Kudchadker, R. J., Wang, J., Bathala, T., Szklaruk, J., Pugh, T. J., ... Frank, S. J. (2016). Development of a magnetic resonance imaging protocol to visualize encapsulated 
contrast agent markers in prostate brachytherapy recipients: initial patient experience. Journal of Contemporary Brachytherapy, 8(3), 235-42. doi:10.5114/jcb.2016.60506

Lim, T. Y., Kudchadker, R. J., Wang, J., Stafford, R. J., MacLellan, C., Rao, A., ... Frank, S. J. (2016). Effect of pulse sequence parameter selection on signal strength in positive-contrast MRI markers for MRI-based prostate postimplant assessment. Medical Physics, 43(7), 4312-4322. doi:10.1118/1.4953635

Lim, T. Y., Stafford, R. J., Kudchadker, R. J., Sankaranarayanapillai, M., Ibbott, G., Rao, A., ... Frank, S. J. (2014a). MRI characterization of cobalt dichloride-N-acetyl cysteine (C4) contrast agent marker for prostate brachytherapy. Physics in Medicine and Biology, 59(10), 2505-16. doi:10.1088/0031-9155/59/10/2505

Lim, T. Y., Stafford, R. J., Kudchadker, R. J., Sankaranarayanapillai, M., Ibbott, G., Rao, A., ... Frank, S. J. (2014b). MRI characterization of cobalt dichloride-N-acetyl cysteine (C4) contrast agent marker for prostate brachytherapy. Institute of Physics and Engineering in Medicine Physics in Medicine and Biology Phys. Med. Biol. Phys. Med. Biol, 59(59), 25052516. doi:10.1088/0031-9155/59/10/2505

Lim, T. Y., Stafford, R. J., Kudchadker, R. J., Sankaranarayanapillai, M., Ibbott, G., Rao, A., ... J, W. G. and J. (2014). MRI characterization of cobalt dichloride-N-acetyl cysteine (C4) contrast agent marker for prostate brachytherapy. Physics in Medicine and Biology, 59(10), 2505-2516. doi:10.1088/0031-9155/59/10/2505

Liu, J., Liu, T., de Rochefort, L., Ledoux, J., Khalidov, I., Chen, W., .. Wang, Y. (2012a). Morphology enabled dipole inversion for quantitative susceptibility mapping using 
structural consistency between the magnitude image and the susceptibility map. NeuroImage, 59(3), 2560-8. doi:10.1016/j.neuroimage.2011.08.082

Liu, J., Liu, T., de Rochefort, L., Ledoux, J., Khalidov, I., Chen, W., .. Wang, Y. (2012b). Morphology enabled dipole inversion for quantitative susceptibility mapping using structural consistency between the magnitude image and the susceptibility map. NeuroImage, 59(3), 2560-2568. doi:10.1016/J.NEUROIMAGE.2011.08.082

Liu, T., Khalidov, I., De Rochefort, L., Spincemaille, P., Liu, J., Tsiouris, A. J., \& Wang, Y. (2011). A novel background field removal method for MRI using projection onto dipole fields (PDF). NMR in Biomedicine, 24, 1129-1136. doi:10.1002/nbm.1670

Liu, T., Liu, J., de Rochefort, L., Spincemaille, P., Khalidov, I., Ledoux, J. R., \& Wang, Y. (2011). Morphology enabled dipole inversion (MEDI) from a single-angle acquisition: comparison with COSMOS in human brain imaging. Magnetic Resonance in Medicine, 66(3), 777-83. doi:10.1002/mrm.22816

Liu, T., Wisnieff, C., Lou, M., Chen, W., Spincemaille, P., \& Wang, Y. (2013). Nonlinear formulation of the magnetic field to source relationship for robust quantitative susceptibility mapping. Magnetic Resonance in Medicine, 69(2), 467-76. doi:10.1002/mrm.24272

Liu, Z., Spincemaille, P., Yao, Y., Zhang, Y., \& Wang, Y. (2018). MEDI+0: Morphology enabled dipole inversion with automatic uniform cerebrospinal fluid zero reference for quantitative susceptibility mapping. Magnetic Resonance in Medicine, 79(5), 2795-2803. doi:10.1002/mrm.26946 
Maglogiannis, I. G. (2007). Emerging artificial intelligence applications in computer engineering : real word AI systems with applications in eHealth, HCI, information retrieval and pervasive technologies. IOS Press.

Manogaran, G., Vijayakumar, V., Varatharajan, R., Malarvizhi Kumar, P., Sundarasekar, R., \& Hsu, C.-H. (2017). Machine Learning Based Big Data Processing Framework for Cancer Diagnosis Using Hidden Markov Model and GM Clustering. Wireless Personal Communications, 1-18. doi:10.1007/s11277-017-5044-Z

Martin, G. V., Ma, J., Kudchadker, R. J., Wang, J., Bruno, T. L., Blanchard, P., \& Frank, S. J. (2017). Automated Prostate Brachytherapy Seed Detection on Post-Implant MRI Using Novel Martin Algorithm. Brachytherapy, 16(3), S57. doi:10.1016/J.BRACHY.2017.04.094

Martin, G. V., Pugh, T. J., Mahmood, U., Kudchadker, R. J., Wang, J., Bruno, T. L., ... Frank, S. J. (2017). Permanent prostate brachytherapy postimplant magnetic resonance imaging dosimetry using positive contrast magnetic resonance imaging markers. Brachytherapy, 16(4), 761-769. doi:10.1016/j.brachy.2017.04.004

Mashouf, S., Safigholi, H., Merino, T., Soliman, A., Ravi, A., Morton, G., \& Song, W. Y. (2016). Sensitivity of clinically relevant dosimetric parameters to contouring uncertainty in postimplant dosimetry of low-dose-rate prostate permanent seed brachytherapy. Brachytherapy, 15(6), 774-779. doi:10.1016/j.brachy.2016.08.013

Matakos, A, Balter, J., \& Cao, Y. (2014). Estimation of geometrically undistorted B 0 inhomogeneity maps. Physics in Medicine and Biology, 59(17), 4945-4959. doi:10.1088/0031-9155/59/17/4945 
Matakos, Antonios, \& Fessler, J. A. (2010). Joint estimation of image and fieldmap in parallel MRI using single-shot acquisitions. In 2010 IEEE International Symposium on Biomedical Imaging: From Nano to Macro (pp. 984-987). IEEE. doi:10.1109/ISBI.2010.5490153

Mitina, N., Christie, D., Hill, B., Middlebrook, N., \& Nadezhdin, N. (2016). Which patients benefit from post-implant $\mathrm{CT}$ dosimetry after real-time intraoperative planning for low dose rate prostate brachytherapy? Case series and systematic literature review. Journal of Medical Imaging and Radiation Oncology, 60(2), 260-267. doi:10.1111/1754-9485.12413

Nakano, M., Yorozu, A., Saito, S., Sugawara, A., Maruo, S., Kojima, S., ... Yamanaka, H. (2015). Seed migration after transperineal interstitial prostate brachytherapy by using loose seeds: Japanese prostate cancer outcome study of permanent iodine- 125 seed implantation (J-POPS) multi-institutional cohort study. Radiation Oncology, 10(1), 228. doi:10.1186/s13014-015-0532-3

Nasrabadi, N. M. (2007). Pattern Recognition and Machine Learning. Journal of Electronic Imaging, 16(4), 049901. doi:10.1117/1.2819119

Nosrati, R., Paudel, M., Ravi, A., Pejovic-Milic, A., Morton, G., \& Stanisz, G. J. (2019). Potential applications of the quantitative susceptibility mapping (QSM) in MR-guided radiation therapy. Physics in Medicine \& Biology, 64(14), 145013. doi:10.1088/1361$6560 / \mathrm{ab} 2623$

Nosrati, R., Soliman, A., Safigholi, H., Hashemi, M., Wronski, M., Morton, G., .. Song, W. Y. (2018). MRI-based automated detection of implanted low dose rate (LDR) brachytherapy seeds using quantitative susceptibility mapping (QSM) and unsupervised machine learning 
(ML). Radiotherapy and Oncology, 129(3), 540-547. doi:10.1016/j.radonc.2018.09.003

Ohashi, T., Momma, T., Yamashita, S., Nagatsuma, K., Kanai, K., Kitagawa, K., ... Shigematsu, N. (2012a). Impact of MRI-based postimplant dosimetric assessment in prostate brachytherapy using contrast-enhanced T1-weighted images. Brachytherapy, 11(6), 468-75. doi:10.1016/j.brachy.2011.12.010

Ohashi, T., Momma, T., Yamashita, S., Nagatsuma, K., Kanai, K., Kitagawa, K., ... Shigematsu, N. (2012b). Impact of MRI-based postimplant dosimetric assessment in prostate brachytherapy using contrast-enhanced T1-weighted images. Brachytherapy, 11(6), 468475. doi:10.1016/j.brachy.2011.12.010

Orio, P. F., Merrick, G. S., Grimm, P., Blasko, J., Sylvester, J., Allen, Z. A., ... Chaudhry, U.-H. (2008). Effects of the Time Interval Between Prostate Brachytherapy and Postimplant Dosimetric Evaluation in Community Practice. American Journal of Clinical Oncology, 31(6), 523-531. doi:10.1097/COC.0b013e31816fc95a

Park, H.-S., \& Jun, C.-H. (2009). A simple and fast algorithm for K-medoids clustering. Expert Systems with Applications, 36(2), 3336-3341. doi:10.1016/j.eswa.2008.01.039

Perez-Calatayud, J., Kuipers, F., Ballester, F., Granero, D., Richart, J., Rodriguez, S., ... Santos, M. (2009). Exclusive MRI-based tandem and colpostats reconstruction in gynaecological brachytherapy treatment planning. Radiotherapy and Oncology, 91(2), 181-186. doi:10.1016/J.RADONC.2008.09.004

Polo, A., Cattani, F., Vavassori, A., Origgi, D., Villa, G., Marsiglia, H., .. Orecchia, R. (2004a). 
MR and CT image fusion for postimplant analysis in permanent prostate seed implants. International Journal of Radiation Oncology, Biology, Physics, 60(5), 1572-9. doi:10.1016/j.ijrobp.2004.08.033

Polo, A., Cattani, F., Vavassori, A., Origgi, D., Villa, G., Marsiglia, H., ... Orecchia, R. (2004b). MR and CT image fusion for postimplant analysis in permanent prostate seed implants. International Journal of Radiation Oncology*Biology*Physics, 60(5), 1572-1579. doi:10.1016/j.ijrobp.2004.08.033

Prete, J. J., Prestidge, B. R., Bice, W. S., Dubois, D. F., \& Hotchkiss, L. A. (1998). Comparison of MRI- and CT-based post-implant dosimetric analysis of transperineal interstitial permanent prostate brachytherapy. Radiation Oncology Investigations, 6(2), 90-96.

Rehman, I., Smith, R., Hench, L. L., \& Bonfield, W. (1995). Structural evaluation of human and sheep bone and comparison with synthetic hydroxyapatite by FT-Raman spectroscopy. Journal of Biomedical Materials Research, 29(10), 1287-94. doi:10.1002/jbm.820291016

Rivard, M. J., Coursey, B. M., Dewerd, L. A., Hanson, W. F., Huq, M. S., Ibbott, G. S., ... Williamson, J. F. (2004). Update of AAPM Task Group No. 43 Report: A revised AAPM protocol for brachytherapy dose calculations. Medical Physics, 31(3), 633-647. doi:10.1118/1.1646040,

Ronneberger, O., Fischer, P., \& Brox, T. (2015). U-Net: Convolutional Networks for Biomedical Image Segmentation. Medical Image Computing and Computer-Assisted Intervention, MICCAI 2015. Lecture Notes in Computer Science, 9351, 234-241. 
Rosenthal, S. A., Bittner, N. H. J., Beyer, D. C., Demanes, D. J., Goldsmith, B. J., Horwitz, E. M., ... American College of Radiology. (2011). American Society for Radiation Oncology (ASTRO) and American College of Radiology (ACR) Practice Guideline for the Transperineal Permanent Brachytherapy of Prostate Cancer. International Journal of Radiation Oncology*Biology*Physics, 79(2), 335-341. doi:10.1016/j.ijrobp.2010.08.045

Rousseeuw, P. J. (1987). Silhouettes: A graphical aid to the interpretation and validation of cluster analysis. Journal of Computational and Applied Mathematics, 20, 53-65. doi:10.1016/0377-0427(87)90125-7

Safigholi, H., Sardari, D., Karimi Jashni, S., Mahdavi, S. R., \& Meigooni, A. S. (2013). An analytical model to determine interseed attenuation effect in low-dose-rate brachytherapy. Journal of Applied Clinical Medical Physics, 14(3), 4226.

Saito, M., \& Sagara, S. (2017). A simple formulation for deriving effective atomic numbers via electron density calibration from dual-energy CT data in the human body. Medical Physics, 44(6), 2293-2303. doi:10.1002/mp.12176

Salembier, C., Lavagnini, P., Nickers, P., Mangili, P., Rijnders, A., Polo, A., ... Hoskin, P. (2007). Tumour and target volumes in permanent prostate brachytherapy: A supplement to the ESTRO/EAU/EORTC recommendations on prostate brachytherapy. Radiotherapy and Oncology, 83(1), 3-10. doi:10.1016/j.radonc.2007.01.014

Sander, J., Ester, M., Kriegel, H.-P., \& Xu, X. (1998). Density-Based Clustering in Spatial Databases: The Algorithm GDBSCAN and Its Applications. Data Mining and Knowledge Discovery, 2(2), 169-194. doi:10.1023/A:1009745219419 
Schenck, J. F. (1996). The role of magnetic susceptibility in magnetic resonance imaging: MRI magnetic compatibility of the first and second kinds. Medical Physics, 23(6), 815-850. doi:10.1118/1.597854

Schofield, M. A., \& Zhu, Y. (2003). Fast phase unwrapping algorithm for interferometric applications. Optics Letters, 28(14), 1194-6.

Segedin, B., \& Petric, P. (2016). Uncertainties in target volume delineation in radiotherapy - are they relevant and what can we do about them? Radiology and Oncology, 50(3), 254-62. doi:10.1515/raon-2016-0023

Shi, C., Xie, G., Zhang, Y., Zhang, X., Chen, M., Su, S., ... Ji, J. (2017). Accelerated susceptibility-based positive contrast imaging of MR compatible metallic devices based on modified fast spin echo sequences. Physics in Medicine and Biology, 62(7), 2505-2520. doi:10.1088/1361-6560/aa5fa3

Skowronek, J. (2013). Low-dose-rate or high-dose-rate brachytherapy in treatment of prostate cancer - between options. Journal of Contemporary Brachytherapy, 5(1), 33-41. doi: $10.5114 /$ jcb.2013.34342

Stock, R. G., \& Stone, N. N. (2002). Importance of post-implant dosimetry in permanent prostate brachytherapy. European Urology, 41(4), 434-9.

Stuber, M., Gilson, W. D., Schär, M., Kedziorek, D. A., Hofmann, L. V, Shah, S., ... Kraitchman, D. L. (2007). Positive contrast visualization of iron oxide-labeled stem cells using inversion-recovery with ON-resonant water suppression (IRON). Magnetic 
Resonance in Medicine, 58(5), 1072-7. doi:10.1002/mrm.21399

Su, Y., Davis, B. J., Furutani, K. M., Herman, M. G., \& Robb, R. A. (2007). Dosimetry accuracy as a function of seed localization uncertainty in permanent prostate brachytherapy: increased seed number correlates with less variability in prostate dosimetry. Physics in Medicine and Biology, 52(11), 3105-3119. doi:10.1088/0031-9155/52/11/012

Suh, J. H., Gardner, J. M., Kee, K. H., Shen, S., Ayala, A. G., \& Ro, J. Y. (2008). Calcifications in prostate and ejaculatory system: a study on 298 consecutive whole mount sections of prostate from radical prostatectomy or cystoprostatectomy specimens. Annals of Diagnostic Pathology, 12(3), 165-170. doi:10.1016/j.anndiagpath.2007.07.001

Suntharalingam, N., Podgorsak, E., \& TÖLLI, H. (2005). Brachytherapy: physical and clinical aspects. Podgorsak EB. Radiation ....

Tanaka, O., Hayashi, S., Matsuo, M., Sakurai, K., Nakano, M., Maeda, S., ... Hoshi, H. (2006a). Comparison of MRI-based and CT/MRI fusion-based postimplant dosimetric analysis of prostate brachytherapy. International Journal of Radiation Oncology, Biology, Physics, 66(2), 597-602. doi:10.1016/j.ijrobp.2006.06.023

Tanaka, O., Hayashi, S., Matsuo, M., Sakurai, K., Nakano, M., Maeda, S., ... Hoshi, H. (2006b). Comparison of MRI-based and CT/MRI fusion-based postimplant dosimetric analysis of prostate brachytherapy. International Journal of Radiation Oncology*Biology*Physics, 66(2), 597-602. doi:10.1016/J.IJROBP.2006.06.023

Tanaka, O., Hayashi, S., Matsuo, M., Sakurai, K., Nakano, M., Maeda, S., ... Hoshi, H. (2006c). 
Comparison of MRI-based and CT/MRI fusion-based postimplant dosimetric analysis of prostate brachytherapy. International Journal of Radiation Oncology*Biology*Physics, 66(2), 597-602. doi:10.1016/j.ijrobp.2006.06.023

Tanderup, K., Viswanathan, A. N., Kirisits, C., \& Frank, S. J. (2014). Magnetic resonance image guided brachytherapy. Seminars in Radiation Oncology, 24(3), 181-91. doi:10.1016/j.semradonc.2014.02.007

Venselaar Jack, Meigooni Ali S., Baltas Dimos, H. P. J. (2012). Comprehensive Brachytherapy: Physical and Clinical Aspects.

Volume Magnetic Susceptibility for all the elements in the Periodic Table. (n.d.). Retrieved February 15, 2019, from http://periodictable.com/Properties/A/VolumeMagneticSusceptibility.v.log.html

Wang, H., Balter, J., \& Cao, Y. (2013). Patient-induced susceptibility effect on geometric distortion of clinical brain MRI for radiation treatment planning on a 3T scanner. Physics in Medicine and Biology, 58(3), 465-477. doi:10.1088/0031-9155/58/3/465

Wang, S., \& Summers, R. M. (2012). Machine learning and radiology. Medical Image Analysis, 16(5), 933-951. doi:10.1016/J.MEDIA.2012.02.005

Wang, Yi, \& Liu, T. (2015). Quantitative susceptibility mapping (QSM): Decoding MRI data for a tissue magnetic biomarker. Magnetic Resonance in Medicine, 73(1), 82-101. doi: $10.1002 / \mathrm{mrm} .25358$

Wang, Yongjin, Sankreacha, R., Al-Hebshi, A., Loblaw, A., \& Morton, G. (2006). Comparative 
study of dosimetry between high-dose-rate and permanent prostate implant brachytherapies in patients with prostate adenocarcinoma. Brachytherapy, 5(4), 251-255. doi:10.1016/j.brachy.2006.08.006

Wills, R., Lowe, G., Inchley, D., Anderson, C., Beenstock, V., \& Hoskin, P. (2010). Applicator reconstruction for HDR cervix treatment planning using images from $0.35 \mathrm{~T}$ open $\mathrm{MR}$ scanner. Radiotherapy and Oncology, 94(3), 346-352.

doi:10.1016/J.RADONC.2009.10.015

Wu, B., Li, W., Guidon, A., \& Liu, C. (2012). Whole brain susceptibility mapping using compressed sensing. Magnetic Resonance in Medicine, 67(1), 137-147. doi: $10.1002 / \mathrm{mrm} .23000$

Yang, X., Zhan, S., Xie, D., Zhao, H., \& Kurihara, T. (2017). Hierarchical prostate MRI segmentation via level set clustering with shape prior. Neurocomputing, 257, 154-163. doi:10.1016/J.NEUCOM.2016.12.071

Yu, Y., Anderson, L. L., Li, Z., Mellenberg, D. E., Nath, R., Schell, M. C., ... Blasko, J. C. (1999). Permanent prostate seed implant brachytherapy: report of the American Association of Physicists in Medicine Task Group No. 64. Medical Physics, 26(10), 2054-2076. doi: $10.1118 / 1.598721$

Zacharaki, E. I., Wang, S., Chawla, S., Soo Yoo, D., Wolf, R., Melhem, E. R., \& Davatzikos, C. (2009). Classification of brain tumor type and grade using MRI texture and shape in a machine learning scheme. Magnetic Resonance in Medicine, 62(6), 1609-1618. doi:10.1002/mrm.22147 
Zaorsky, N. G., Davis, B. J., Nguyen, P. L., Showalter, T. N., Hoskin, P. J., Yoshioka, Y., ... Horwitz, E. M. (2017). The evolution of brachytherapy for prostate cancer. Nature Reviews Urology, 14(7), 415-439. doi:10.1038/nrurol.2017.76

Zhang, H., Baker, C., McKinsey, R., \& Meigooni, A. (2005). Dose verification with Monte Carlo technique for prostate brachytherapy implants with 125I sources. Medical Dosimetry, 30(2), 85-91. doi:10.1016/j.meddos.2005.01.003

Zhou, D., Liu, T., Spincemaille, P., \& Wang, Y. (2014). Background field removal by solving the Laplacian boundary value problem. NMR in Biomedicine, 27(3), 312-319. doi: $10.1002 / \mathrm{nbm} .3064$

Zhou, K., Zaitsev, M., \& Bao, S. (2009). Reliable two-dimensional phase unwrapping method using region growing and local linear estimation. Magnetic Resonance in Medicine, 62(4), 1085-1090. doi:10.1002/mrm.22074

Zijlstra, F., Moerland, M. A., van der Voort van Zyp, J. R. N., Noteboom, J. L., Viergever, M. A., \& Seevinck, P. R. (2017). Challenges in MR-only seed localization for postimplant dosimetry in permanent prostate brachytherapy. Medical Physics, 44(10), 5051-5060. doi:10.1002/mp. 12505 